- Supporting Information -

\title{
Nucleophilic Reactivities of Thiophenolates
}

\author{
Patrick M. Jüstel, Cedric D. Pignot, Armin R. Ofial* \\ Department Chemie, Ludwig-Maximilians-Universität München, \\ Butenandtstraße 5-13, 81377 München, Germany \\ ofial@Imu.de
}

Table of Contents

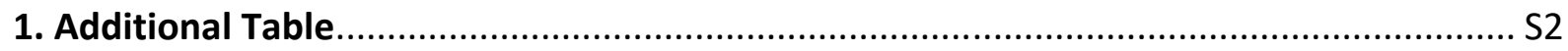

2. UV-Vis Spectra of Thiophenolates 1 and Quinone Methides 2 in DMSO ........................S3

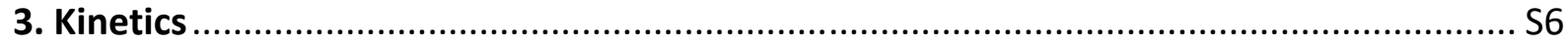

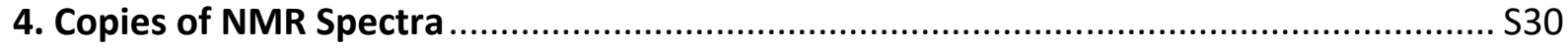

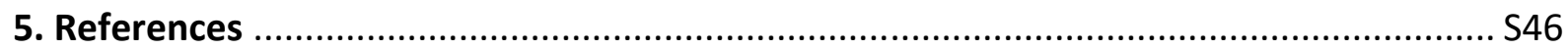




\section{Additional Table}

Table S1. Kinetic and Thermodynamic Data for the Reactions of Thiophenolates 1

\begin{tabular}{|c|c|c|c|c|c|c|c|}
\hline $\mathrm{ArS}^{-}$ & $N / s_{N}$ & $\begin{array}{l}\log k_{2}{ }^{\text {exptl }} \\
(\mathbf{1}+\mathbf{2} \mathbf{c})^{a}\end{array}$ & $\begin{array}{l}\text { Hammett } \\
\sigma_{\mathrm{p}}^{-} \text {or } \sigma_{\mathrm{m}}{ }^{b}\end{array}$ & $\begin{array}{l}\mathrm{p} K_{\mathrm{aH}} \\
(\mathrm{DMSO})^{\mathrm{c}}\end{array}$ & $\begin{array}{l}\left(\log k_{2}\right) / \mathrm{s}_{\mathrm{N}} \\
(1+n \mathrm{BuCl})^{d}\end{array}$ & $\begin{array}{l}\text { Phenolate lons } \\
\mathrm{ArO}^{-}\end{array}$ & $\begin{array}{l}\log k_{2} \\
\left(\mathrm{ArO}^{-}+2 \mathrm{c}\right)^{e}\end{array}$ \\
\hline $1 a$ & $24.97 / 0.68$ & 5.88 & -0.26 & 11.19 & -1.00 & $p-\mathrm{MeO}-\mathrm{C}_{6} \mathrm{H}_{4} \mathrm{O}^{-}$ & 3.18 \\
\hline $1 b$ & $24.35 / 0.69$ & 5.70 & -0.17 & 10.82 & & $p-\mathrm{Me}-\mathrm{C}_{6} \mathrm{H}_{4} \mathrm{O}^{-}$ & 2.58 \\
\hline 1c & $23.36 / 0.74$ & 5.35 & 0 & 10.28 & -1.37 & $\mathrm{C}_{6} \mathrm{H}_{5} \mathrm{O}^{-}$ & 2.66 \\
\hline $1 \mathbf{j}$ & $22.55 / 0.83$ & 5.29 & 0.12 & 9.53 & -1.60 & & \\
\hline $1 d$ & $22.80 / 0.78$ & 5.17 & 0.25 & 8.98 & -1.88 & & \\
\hline $1 e$ & $22.50 / 0.78$ & 4.96 & 0.37 & 8.57 & & & \\
\hline $1 f$ & $21.75 / 0.86$ & 4.75 & 0.43 & 8.09 & -2.24 & & \\
\hline $1 g$ & $21.30 / 0.86$ & 4.44 & 0.65 & & & $p-\mathrm{F}_{3} \mathrm{C}-\mathrm{C}_{6} \mathrm{H}_{4} \mathrm{O}^{-}$ & 1.81 \\
\hline $1 \mathrm{~h}$ & $19.71 / 0.86$ & 3.07 & 0.86 & & & & \\
\hline $1 \mathbf{i}$ & $18.92 / 0.87$ & 2.40 & 1.27 & 5.5 & -3.55 & $p-\mathrm{O}_{2} \mathrm{~N}-\mathrm{C}_{6} \mathrm{H}_{4} \mathrm{O}^{-}$ & -1.38 \\
\hline
\end{tabular}

${ }^{a}$ Data from Table 1 (this work). ${ }^{b}$ Hammett substituent constants $\sigma_{\mathrm{p}}{ }^{-}$or $\sigma_{\mathrm{m}}$ from ref S1. ${ }^{c}$ Acidity constants for thiophenols in DMSO; from ref S2. ${ }^{d}$ Calculated with second-order rate constants $k_{2}$ for the reactions of $\operatorname{ArS}^{-}(\mathbf{1})$ with $n$-butyl chloride in DMSO at $25^{\circ} \mathrm{C}$ from ref S2. ${ }^{e}$ Calculated from secondorder rate constants $k_{2}$ in DMSO at $20^{\circ} \mathrm{C}$; ref S3. 


\section{UV-Vis Spectra of Thiophenolates 1 and Quinone Methides $\mathbf{2}$ in DMSO}

\subsection{UV-Vis Spectra of Thiophenolates 1}

A DMSO solution of the thiophenol 1-H was deprotonated (by $\mathrm{NaH}$ or $\mathrm{KOtBu}$ ) and added in multiple steps to a flask filled with DMSO. The increasing absorptions of the DMSO solutions were followed by utilizing a diode array photometer (J\&M TIDAS). In accord with the Lambert-Beer law, molar absorption coefficients $\varepsilon_{\max }$ (at $\lambda_{\max }$ ) were determined from the slope of the linear relationship of the absorbance (at $\lambda_{\max }$ ) with [1]. The depicted UV-vis spectra were then normalized relative to the extinction coefficient at $\lambda_{\max }$.

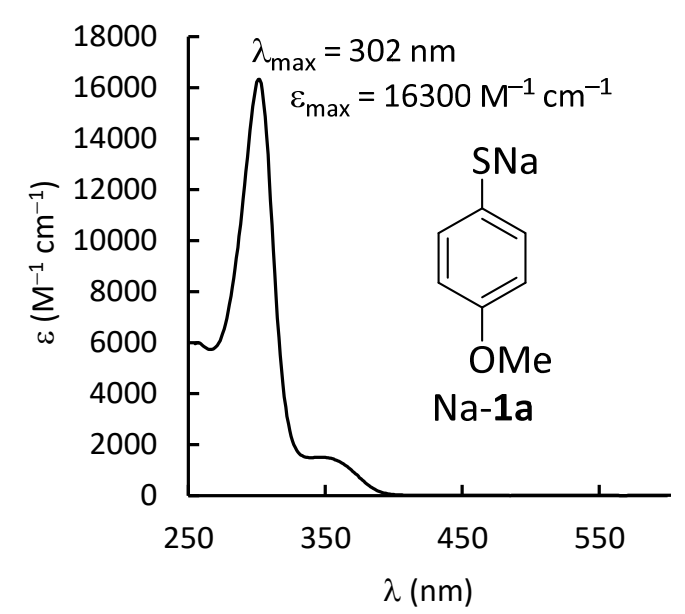

Figure S1. UV-vis spectrum of Na-1a in DMSO.

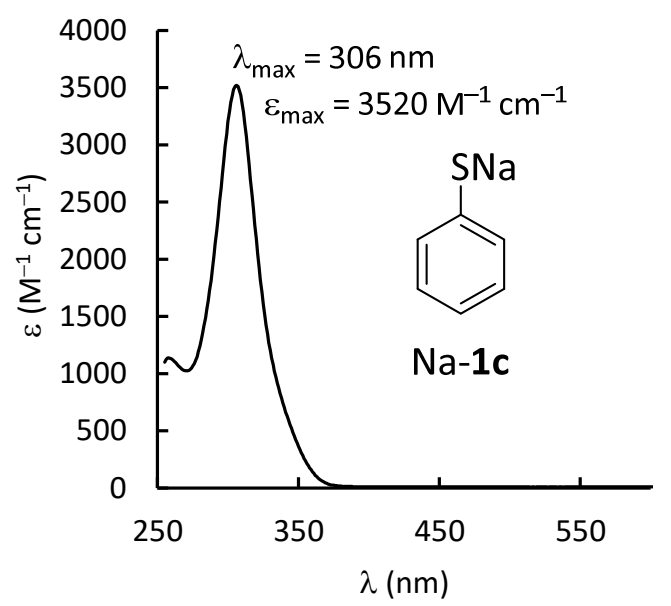

Figure S3. UV-vis spectrum of $\mathrm{Na}-1 \mathrm{c}$ in DMSO.

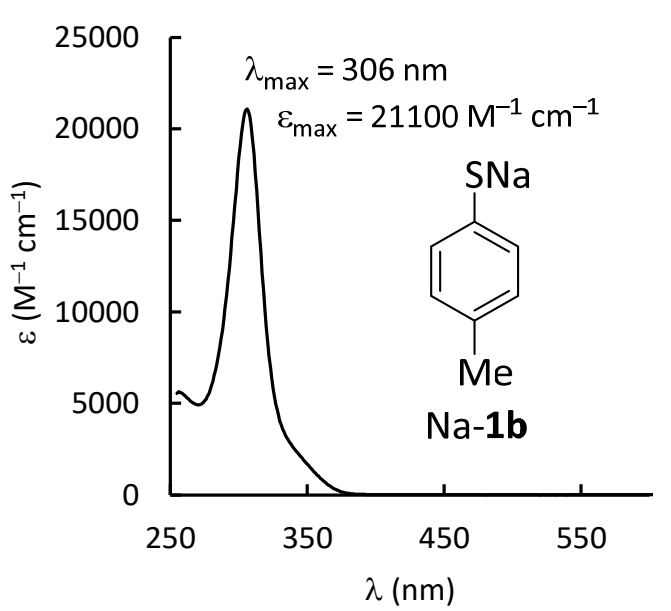

Figure S2. UV-vis spectrum of $\mathrm{Na-1b}$ in DMSO.

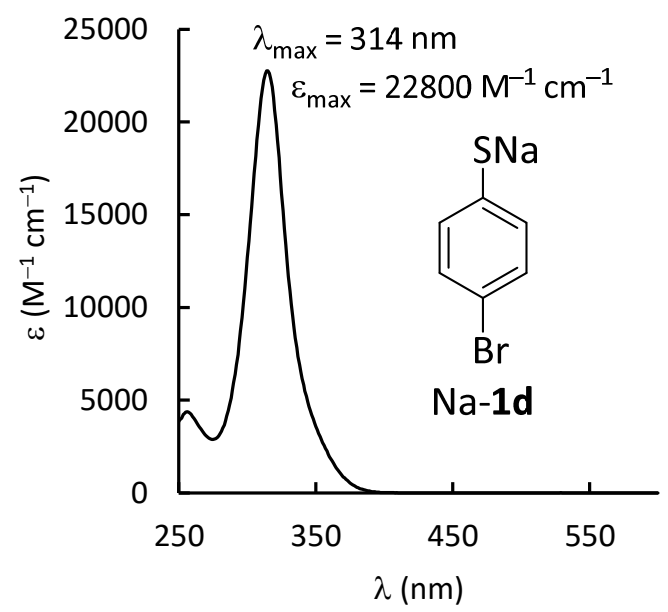

Figure S4. UV-vis spectrum of Na-1d in DMSO. 


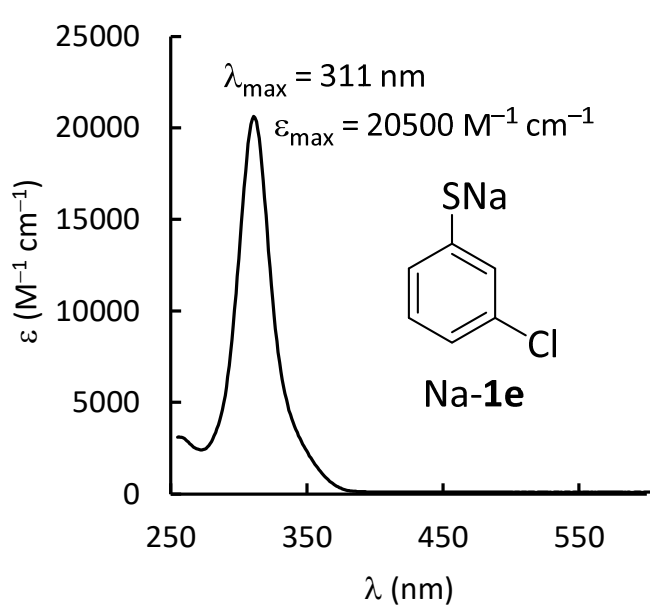

Figure S5. UV-vis spectrum of $\mathrm{Na}-1 \mathrm{e}$ in DMSO.

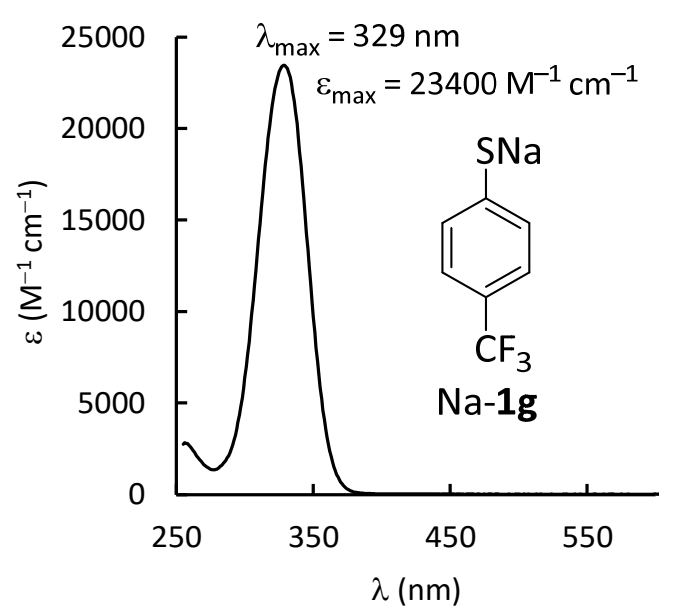

Figure S7. UV-vis spectrum of $\mathrm{Na}-\mathbf{1 g}$ in DMSO.

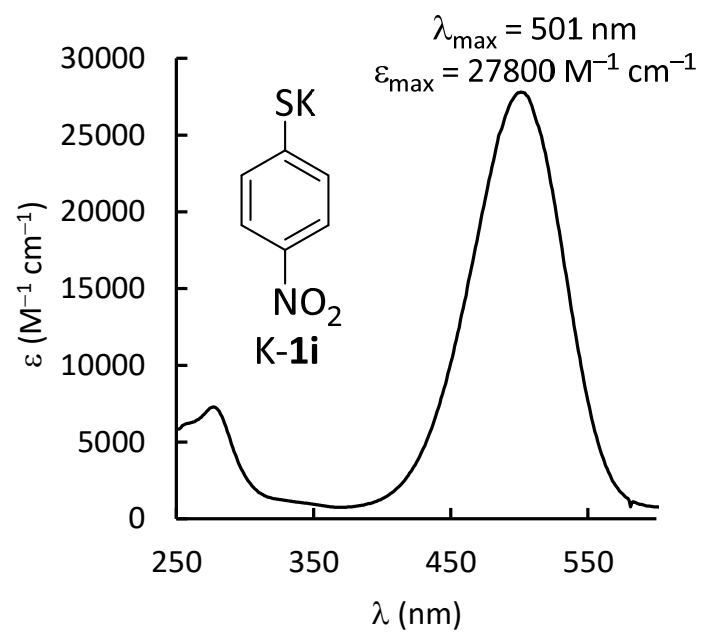

Figure S9. UV-vis spectrum of $\mathrm{K}-\mathbf{1 i}$ in DMSO.

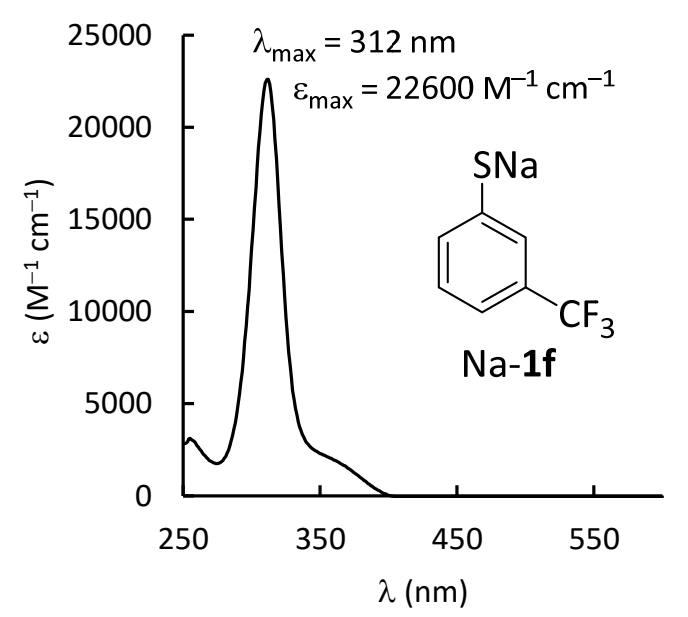

Figure S6. UV-vis spectrum of Na-1f in DMSO.

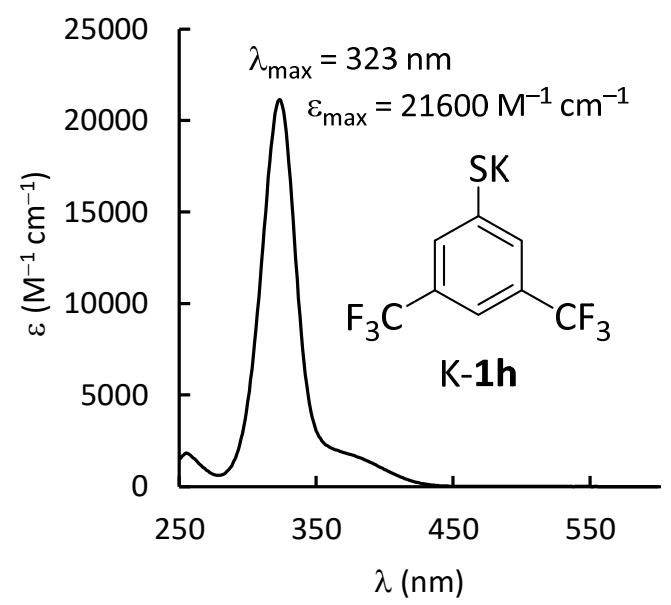

Figure S8. UV-vis spectrum of K-1h in DMSO.

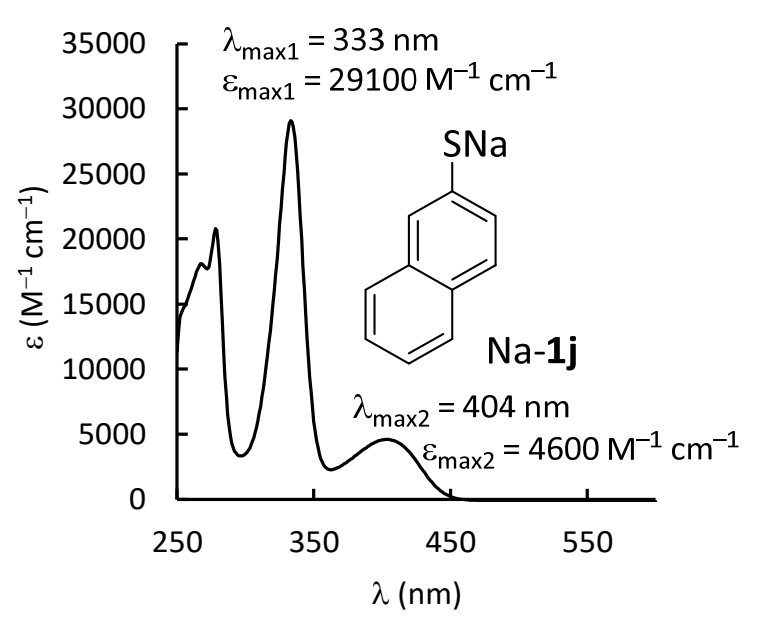

Figure S10. UV-vis spectrum of $\mathrm{Na}-\mathbf{1} \mathbf{j}$ in DMSO. 


\subsection{UV-Vis Spectra of Quinone Methides 2}

A DMSO solution of the quinone methide $\mathbf{2}$ was added in multiple steps to a flask filled with DMSO. The increasing absorptions of the DMSO solutions were followed by utilizing a diode array photometer (J\&M TIDAS). In accord with the Lambert-Beer law, extinction coefficients at $\lambda_{\max }$ were determined from the slope of the linear relationship of the absorbance (at $\lambda_{\max }$ ) with [2]. The depicted UV-vis spectra were then normalized relative to the extinction coefficient at $\lambda_{\max }$.

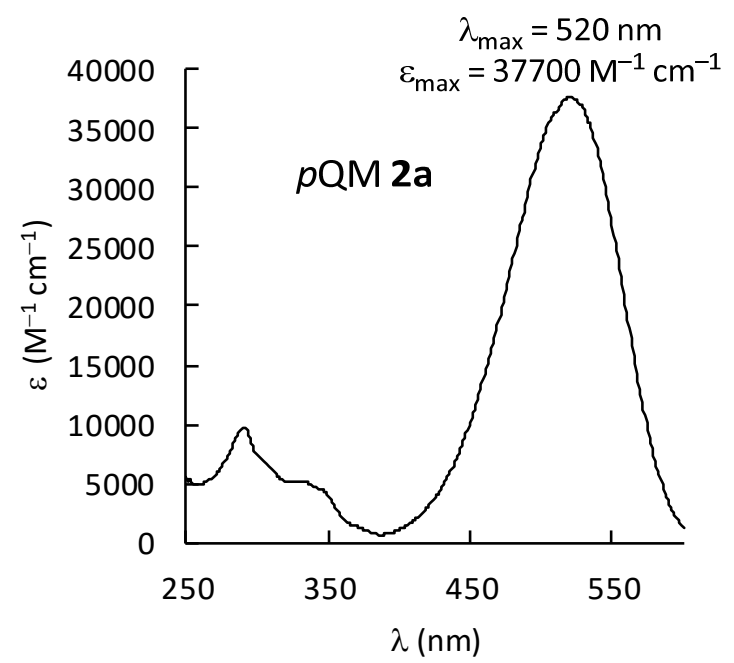

Figure S11. UV-vis spectrum of 2a in DMSO.

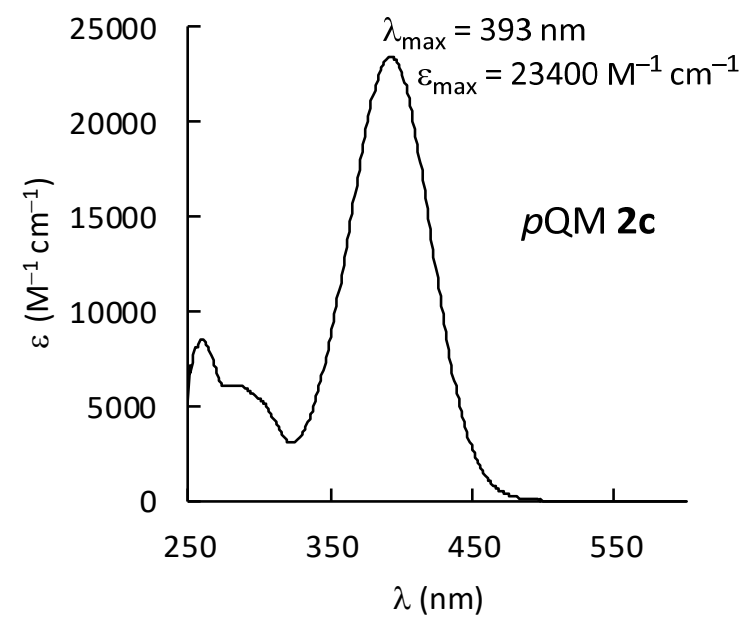

Figure S13. UV-vis spectrum of $2 \mathrm{c}$ in DMSO.

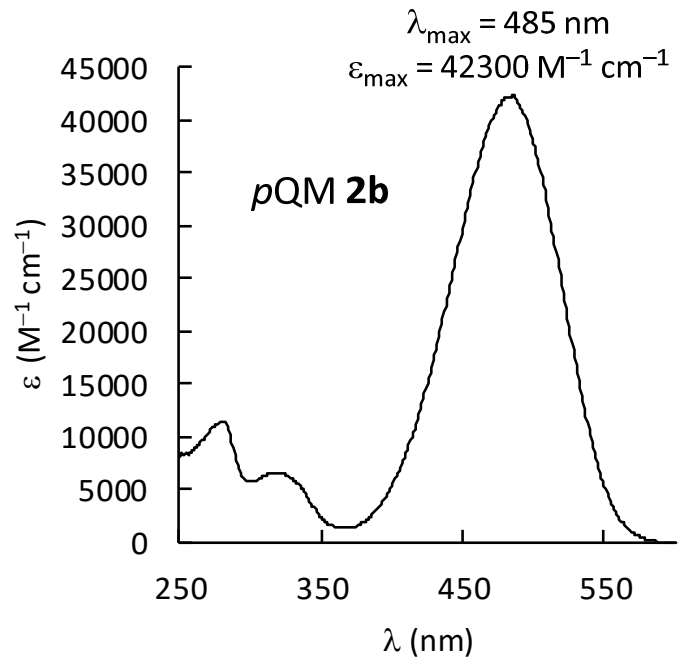

Figure S12. UV-vis spectrum of $\mathbf{2 b}$ in DMSO.

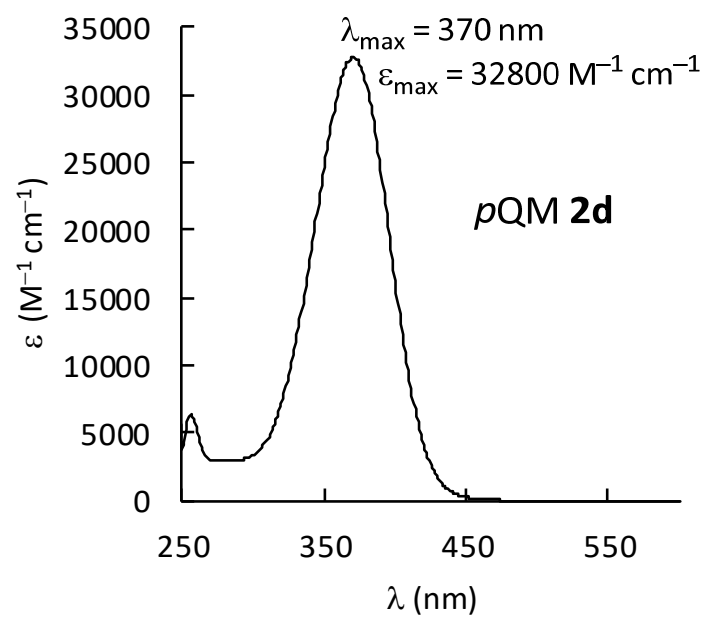

Figure S14. UV-vis spectrum of $\mathbf{2 d}$ in DMSO.

UV-vis spectral data for $p Q M s \mathbf{2 e}$ and $\mathbf{2 f}$ (in DMSO) were reported in ref S4:

For 2e: $\lambda_{\max }=354 \mathrm{~nm}, \varepsilon_{\max }=31600 \mathrm{M}^{-1} \mathrm{~cm}^{-1}$
For 2f: $\lambda_{\max }=374 \mathrm{~nm}, \varepsilon_{\max }=30100 \mathrm{M}^{-1} \mathrm{~cm}^{-1}$ 


\section{Kinetics}

\section{Kinetics at variable thiophenol concentrations}

Table S2. Quinone methide 2c and potassium 4-methoxythiophenolate (K-1a) in DMSO (detection at $393 \mathrm{~nm}$ ) with variable $1 \mathrm{a}-\mathrm{H}$ concentration

\begin{tabular}{lllll}
\hline$\#$ & {$[2 \mathrm{c}]_{0}, \mathrm{M}$} & {$[1 \mathrm{a}]_{0}, \mathrm{M}$} & {$[1 \mathrm{a}-\mathrm{H}]_{0}, \mathrm{M}$} & $k_{\mathrm{obs}, \mathrm{s}^{-1}}$ \\
\hline 1 & $5.00 \times 10^{-5}$ & $1.00 \times 10^{-3}$ & $2.50 \times 10^{-3}$ & $858 \pm 7$ \\
2 & $5.00 \times 10^{-5}$ & $1.00 \times 10^{-3}$ & $5.00 \times 10^{-3}$ & $911 \pm 5$ \\
3 & $5.00 \times 10^{-5}$ & $1.00 \times 10^{-3}$ & $1.00 \times 10^{-2}$ & $909 \pm 6$ \\
4 & $5.00 \times 10^{-5}$ & $1.00 \times 10^{-3}$ & $2.00 \times 10^{-2}$ & $876 \pm 6$ \\
\hline
\end{tabular}

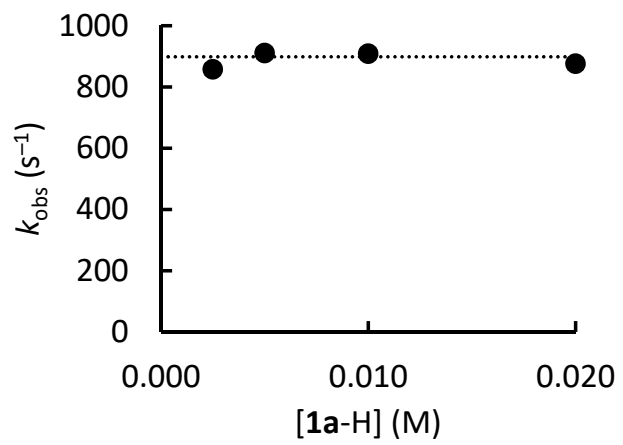

The dashed line shows the average $k_{\mathrm{obs}}$ of entries \#2, \#3, and \#4, that is, $k_{\mathrm{obs}}=(899 \pm 16) \mathrm{s}^{-1}$.

Table S3. Quinone methide $\mathbf{2 c}$ and sodium thiophenolate ( $\mathrm{Na}-\mathbf{1 c}$ ) in DMSO (detection at $393 \mathrm{~nm}$ ) with variable 1c-H concentration

\begin{tabular}{lllll}
\hline$\#$ & {$[\mathbf{2 c}]_{0}, \mathrm{M}$} & {$[\mathbf{1 c}]_{0}, \mathrm{M}$} & {$[\mathbf{1 c}-\mathrm{H}]_{0}, \mathrm{M}$} & $k_{\mathrm{obs}, \mathrm{s}^{-1}}$ \\
\hline 1 & $5.00 \times 10^{-5}$ & $1.00 \times 10^{-3}$ & $2.50 \times 10^{-4}$ & $128 \pm 1$ \\
2 & $5.00 \times 10^{-5}$ & $1.00 \times 10^{-3}$ & $5.00 \times 10^{-4}$ & $194 \pm 1$ \\
3 & $5.00 \times 10^{-5}$ & $1.00 \times 10^{-3}$ & $1.00 \times 10^{-3}$ & $225 \pm 1$ \\
4 & $5.00 \times 10^{-5}$ & $1.00 \times 10^{-3}$ & $1.50 \times 10^{-3}$ & $224 \pm 1$ \\
5 & $5.00 \times 10^{-5}$ & $1.00 \times 10^{-3}$ & $2.00 \times 10^{-3}$ & $236 \pm 1$ \\
6 & $5.00 \times 10^{-5}$ & $1.00 \times 10^{-3}$ & $5.00 \times 10^{-3}$ & $243 \pm 1$ \\
\hline
\end{tabular}

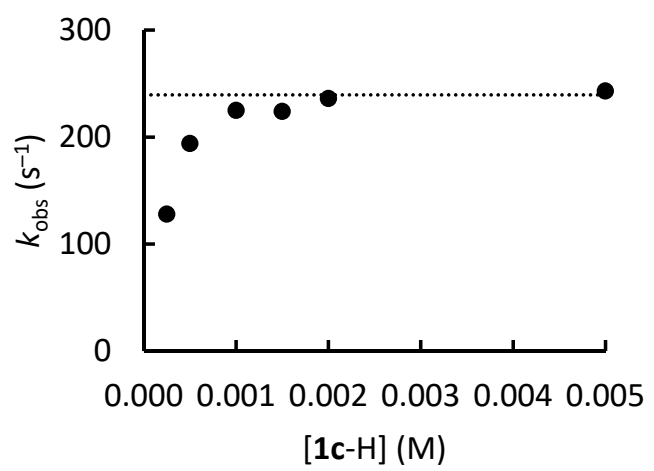

The dashed line shows the average $k_{\mathrm{obs}}$ of entries \#5 and \#6, that is, $k_{\mathrm{obs}}=(240 \pm 4) \mathrm{s}^{-1}$. 
Kinetics of reactions of 4-methoxythiophenolate (1a) with quinone methides 2

Table S4. Quinone methide $\mathbf{2 a}$ and sodium 4-methoxythiophenolate (Na-1a) in DMSO (detection at $521 \mathrm{~nm}$ )

\begin{tabular}{crcc}
\hline$[\mathbf{2 a}]_{0}, \mathrm{M}$ & {$[\mathbf{1} \mathrm{a}]_{0}, \mathrm{M}$} & {$[\mathbf{1 a}-\mathrm{H}]_{0}, \mathrm{M}$} & $k_{\text {obs }}, \mathrm{s}^{-1}$ \\
\hline $5.00 \times 10^{-5}$ & $5.00 \times 10^{-4}$ & $5.00 \times 10^{-3}$ & 30.9 \\
$5.00 \times 10^{-5}$ & $7.50 \times 10^{-4}$ & $5.00 \times 10^{-3}$ & 47.4 \\
$5.00 \times 10^{-5}$ & $1.00 \times 10^{-3}$ & $5.00 \times 10^{-3}$ & 63.4 \\
$5.00 \times 10^{-5}$ & $1.25 \times 10^{-3}$ & $5.00 \times 10^{-3}$ & 82.1 \\
$5.00 \times 10^{-5}$ & $1.50 \times 10^{-3}$ & $5.00 \times 10^{-3}$ & 96.0 \\
\hline
\end{tabular}

$$
k_{2}=6.60 \times 10^{4} \mathrm{M}^{-1} \mathrm{~s}^{-1}
$$

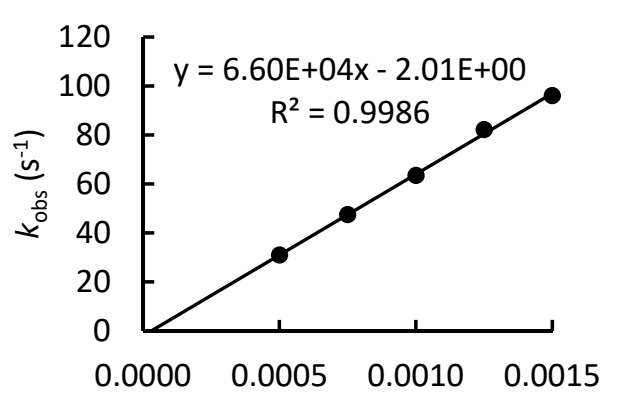

[1a] (M)

Table S5. Quinone methide $\mathbf{2 b}$ and sodium 4-methoxythiophenolate (Na-1a) in DMSO (detection at $486 \mathrm{~nm}$ )

\begin{tabular}{llll}
\hline$[\mathbf{2 b}]_{0}, \mathrm{M}$ & {$[\mathbf{1 a}]_{0}, \mathrm{M}$} & {$[1 \mathrm{a}-\mathrm{H}]_{0}, \mathrm{M}$} & $k_{\mathrm{obs}}, \mathrm{s}^{-1}$ \\
\hline $5.00 \times 10^{-5}$ & $5.00 \times 10^{-4}$ & $5.00 \times 10^{-3}$ & 64.2 \\
$5.00 \times 10^{-5}$ & $7.50 \times 10^{-4}$ & $5.00 \times 10^{-3}$ & 97.5 \\
$5.00 \times 10^{-5}$ & $1.00 \times 10^{-3}$ & $5.00 \times 10^{-3}$ & 130 \\
$5.00 \times 10^{-5}$ & $1.25 \times 10^{-3}$ & $5.00 \times 10^{-3}$ & 169 \\
$5.00 \times 10^{-5}$ & $1.50 \times 10^{-3}$ & $5.00 \times 10^{-3}$ & 199 \\
\hline
\end{tabular}

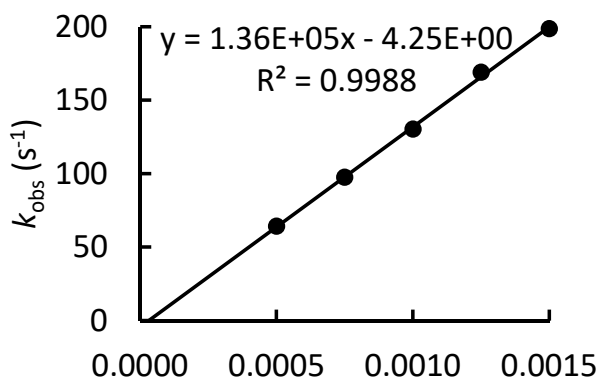

$$
k_{2}=1.36 \times 10^{5} \mathrm{M}^{-1} \mathrm{~s}^{-1}
$$

[1a] (M)

Table S6. Quinone methide $\mathbf{2 c}$ and sodium 4-methoxythiophenolate (Na-1a) in DMSO (detection at $393 \mathrm{~nm}$ )

\begin{tabular}{llll}
\hline$[\mathbf{2 c}]_{0}, \mathrm{M}$ & {$[\mathbf{1 a}]_{0}, \mathrm{M}$} & {$[\mathbf{1 a}-\mathrm{H}]_{0}, \mathrm{M}$} & $k_{\text {obs }}, \mathrm{s}^{-1}$ \\
\hline $5.00 \times 10^{-5}$ & $5.00 \times 10^{-4}$ & $5.00 \times 10^{-3}$ & 480 \\
$5.00 \times 10^{-5}$ & $7.50 \times 10^{-4}$ & $5.00 \times 10^{-3}$ & 693 \\
$5.00 \times 10^{-5}$ & $1.00 \times 10^{-3}$ & $5.00 \times 10^{-3}$ & 895 \\
$5.00 \times 10^{-5}$ & $1.25 \times 10^{-3}$ & $5.00 \times 10^{-3}$ & $1.07 \times 10^{3}$ \\
$5.00 \times 10^{-5}$ & $1.50 \times 10^{-3}$ & $5.00 \times 10^{-3}$ & $1.24 \times 10^{3}$ \\
\hline
\end{tabular}

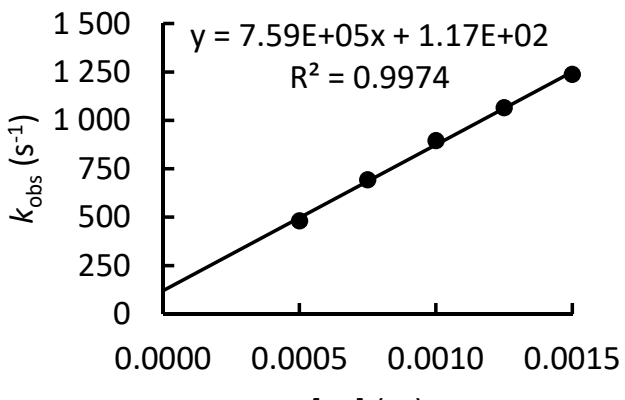

$$
k_{2}=7.59 \times 10^{5} \mathrm{M}^{-1} \mathrm{~s}^{-1}
$$

[1a] (M) 
Table S7. Quinone methide $\mathbf{2} \mathbf{d}$ and potassium 4-methoxythiophenolate (K-1a) in DMSO (detection at $371 \mathrm{~nm}$ )

\begin{tabular}{llll}
\hline$[2 \mathrm{~d}]_{0}, \mathrm{M}$ & {$[1 \mathrm{a}]_{0}, \mathrm{M}$} & {$[1 \mathrm{a}-\mathrm{H}]_{0}, \mathrm{M}$} & $k_{\mathrm{obs}, \mathrm{s}^{-1}}$ \\
\hline $5.00 \times 10^{-5}$ & $1.16 \times 10^{-4}$ & $5.00 \times 10^{-3}$ & 164 \\
$5.00 \times 10^{-5}$ & $2.32 \times 10^{-4}$ & $5.00 \times 10^{-3}$ & 371 \\
$5.00 \times 10^{-5}$ & $3.48 \times 10^{-4}$ & $5.00 \times 10^{-3}$ & 603 \\
$5.00 \times 10^{-5}$ & $4.64 \times 10^{-4}$ & $5.00 \times 10^{-3}$ & 807 \\
\hline
\end{tabular}

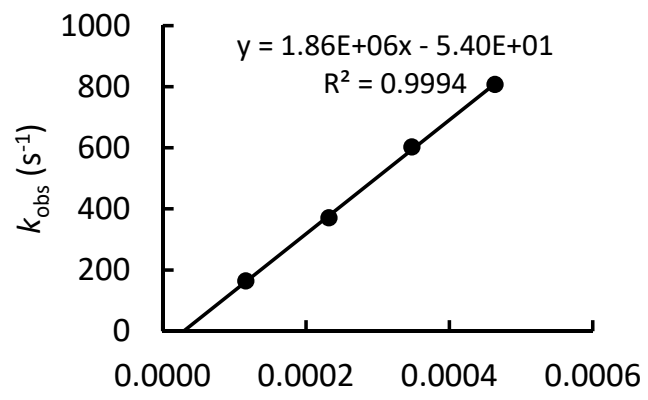

[1a] (M)

$$
k_{2}=1.86 \times 10^{6} \mathrm{M}^{-1} \mathrm{~s}^{-1}
$$

Table S8. Determination of $N$ and $s_{N}$ parameters for 4-methoxythiophenolate (1a) in DMSO.

\begin{tabular}{llll}
\hline $\begin{array}{l}\text { Quinone } \\
\text { methide }\end{array}$ & $E$ & $k_{2}, \mathrm{M}^{-1} \mathrm{~s}^{-1}$ & $\log k_{2}$ \\
\hline $\mathbf{2 a}$ & -17.90 & $6.60 \times 10^{4}$ & 4.82 \\
$\mathbf{2 b}$ & -17.29 & $1.36 \times 10^{5}$ & 5.13 \\
$\mathbf{2 c}$ & -16.11 & $7.59 \times 10^{5}$ & 5.88 \\
$\mathbf{2 d}$ & -15.83 & $1.86 \times 10^{6}$ & 6.27 \\
\hline
\end{tabular}

$N=24.97$

$s_{N}=0.68$

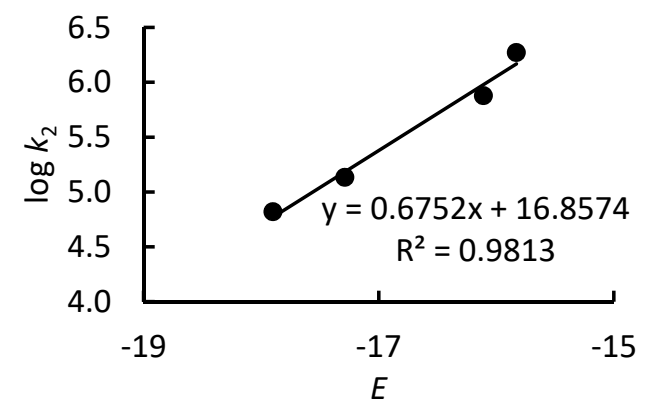




\section{Kinetics of reactions of 4-methylthiophenolate (1b) with quinone methides 2}

Table S9. Quinone methide $\mathbf{2 a}$ and sodium 4-methylthiophenolate (Na-1b) in DMSO (detection at $521 \mathrm{~nm}$ )

\begin{tabular}{llll}
\hline$[\mathbf{2 a}]_{0}, \mathrm{M}$ & {$[\mathbf{1 b}]_{0}, \mathrm{M}$} & {$[\mathbf{1} \mathbf{b}-\mathrm{H}]_{0}, \mathrm{M}$} & $k_{\text {obs }}, \mathrm{s}^{-1}$ \\
\hline $5.00 \times 10^{-5}$ & $5.00 \times 10^{-4}$ & $5.00 \times 10^{-3}$ & 17.1 \\
$5.00 \times 10^{-5}$ & $1.00 \times 10^{-3}$ & $5.00 \times 10^{-3}$ & 31.5 \\
$5.00 \times 10^{-5}$ & $1.50 \times 10^{-3}$ & $5.00 \times 10^{-3}$ & 50.5 \\
$5.00 \times 10^{-5}$ & $2.00 \times 10^{-3}$ & $5.00 \times 10^{-3}$ & 65.9 \\
\hline
\end{tabular}

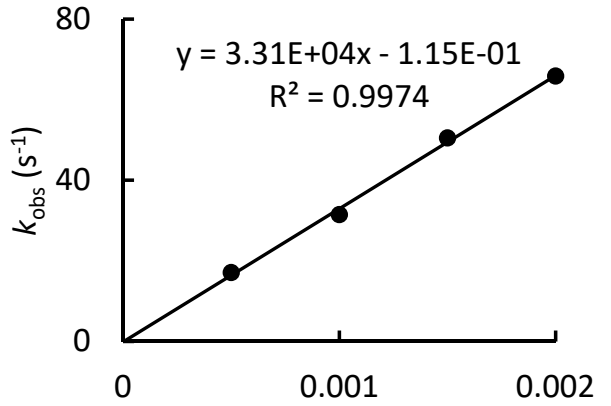

[1b] (M)

$$
k_{2}=3.31 \times 10^{4} \mathrm{M}^{-1} \mathrm{~s}^{-1}
$$

Table S10. Quinone methide $\mathbf{2 b}$ and sodium 4-methylthiophenolate (Na-1b) in DMSO (detection at $486 \mathrm{~nm}$ )

\begin{tabular}{llll}
\hline$[\mathbf{2 b}]_{0}, \mathrm{M}$ & {$[\mathbf{1 b}]_{0}, \mathrm{M}$} & {$[\mathbf{1 b}-\mathrm{H}]_{0}, \mathrm{M}$} & $k_{\mathrm{obs},} \mathrm{s}^{-1}$ \\
\hline $5.00 \times 10^{-5}$ & $5.00 \times 10^{-4}$ & $5.00 \times 10^{-3}$ & 34.2 \\
$5.00 \times 10^{-5}$ & $1.00 \times 10^{-3}$ & $5.00 \times 10^{-3}$ & 64.9 \\
$5.00 \times 10^{-5}$ & $1.50 \times 10^{-3}$ & $5.00 \times 10^{-3}$ & 102 \\
$5.00 \times 10^{-5}$ & $2.00 \times 10^{-3}$ & $5.00 \times 10^{-3}$ & 133 \\
\hline
\end{tabular}

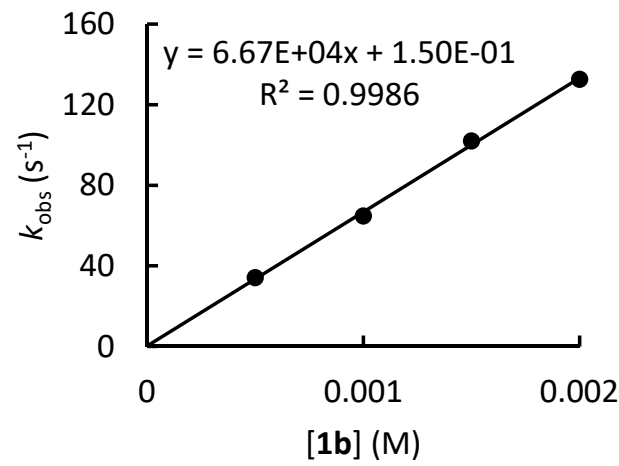

$$
k_{2}=6.67 \times 10^{4} \mathrm{M}^{-1} \mathrm{~s}^{-1}
$$

Table S11. Quinone methide $\mathbf{2 c}$ and sodium 4-methylthiophenolate ( $\mathbf{N a - 1 b}$ ) in DMSO (detection at $393 \mathrm{~nm}$ )

\begin{tabular}{llll}
\hline$[\mathbf{2 c}]_{0}, \mathrm{M}$ & {$[\mathbf{1 b}]_{0}, \mathrm{M}$} & {$[\mathbf{1} \mathbf{b}-\mathrm{H}]_{0}, \mathrm{M}$} & $k_{\text {obs }}, \mathrm{s}^{-1}$ \\
\hline $5.00 \times 10^{-5}$ & $5.00 \times 10^{-4}$ & $5.00 \times 10^{-3}$ & 262 \\
$5.00 \times 10^{-5}$ & $1.00 \times 10^{-3}$ & $5.00 \times 10^{-3}$ & 519 \\
$5.00 \times 10^{-5}$ & $1.50 \times 10^{-3}$ & $5.00 \times 10^{-3}$ & 801 \\
$5.00 \times 10^{-5}$ & $2.00 \times 10^{-3}$ & $5.00 \times 10^{-3}$ & 994 \\
\hline
\end{tabular}

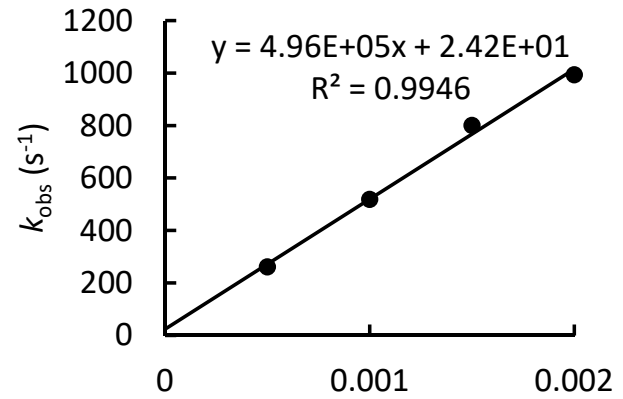

[1b] (M)

$k_{2}=4.96 \times 10^{5} \mathrm{M}^{-1} \mathrm{~s}^{-1}$ 
Table S12. Quinone methide $\mathbf{2 d}$ and potassium 4-methylthiophenolate (K-1b) in DMSO (detection at $371 \mathrm{~nm}$ )

\begin{tabular}{llll}
\hline$[\mathbf{2 d}]_{0}, \mathrm{M}$ & {$[\mathbf{1} \mathbf{b}]_{0}, \mathrm{M}$} & {$[\mathbf{1 b}-\mathrm{H}]_{0}, \mathrm{M}$} & $k_{\mathrm{obs}}, \mathrm{s}^{-1}$ \\
\hline $5.00 \times 10^{-5}$ & $1.34 \times 10^{-4}$ & $5.00 \times 10^{-3}$ & 110 \\
$5.00 \times 10^{-5}$ & $2.67 \times 10^{-4}$ & $5.00 \times 10^{-3}$ & 230 \\
$5.00 \times 10^{-5}$ & $4.01 \times 10^{-4}$ & $5.00 \times 10^{-3}$ & 345 \\
$5.00 \times 10^{-5}$ & $5.35 \times 10^{-4}$ & $5.00 \times 10^{-3}$ & 457 \\
\hline
\end{tabular}

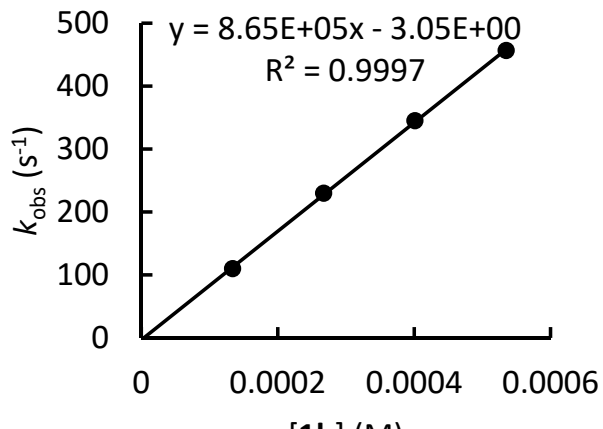

$$
k_{2}=8.65 \times 10^{5} \mathrm{M}^{-1} \mathrm{~s}^{-1}
$$

[1b] (M)

Table S13. Determination of $N$ and $s_{N}$ parameters for 4-methylthiophenolate (1) $\left.\mathbf{b}\right)$ in DMSO.

\begin{tabular}{llll}
\hline $\begin{array}{l}\text { Quinone } \\
\text { methide }\end{array}$ & $E$ & $k_{2}, \mathrm{M}^{-1} \mathrm{~s}^{-1}$ & $\log k_{2}$ \\
\hline 2a & -17.90 & $3.31 \times 10^{4}$ & 4.52 \\
2b & -17.29 & $6.67 \times 10^{4}$ & 4.82 \\
2c & -16.11 & $4.96 \times 10^{5}$ & 5.70 \\
2d & -15.83 & $8.65 \times 10^{5}$ & 5.94 \\
\hline
\end{tabular}

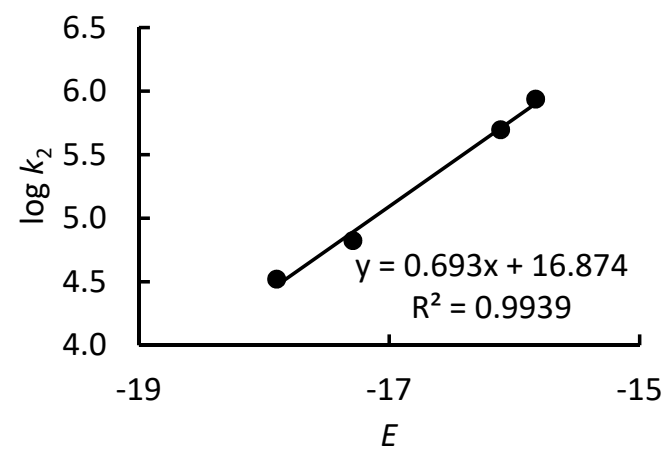

$N=24.35$

$s_{\mathrm{N}}=0.69$ 
Kinetics of reactions of thiophenolate (1c) with quinone methides 2

Table S14. Quinone methide 2a and sodium thiophenolate (Na-1c) in DMSO (detection at $521 \mathrm{~nm}$ )

\begin{tabular}{llll}
\hline$[\mathbf{2 a}]_{0}, \mathrm{M}$ & {$[\mathbf{1 c}]_{0}, \mathrm{M}$} & {$[\mathbf{1 c}-\mathrm{H}]_{0}, \mathrm{M}$} & $k_{\text {obs }}, \mathrm{s}^{-1}$ \\
\hline $5.00 \times 10^{-5}$ & $5.00 \times 10^{-4}$ & $5.00 \times 10^{-3}$ & 9.83 \\
$5.00 \times 10^{-5}$ & $1.00 \times 10^{-3}$ & $5.00 \times 10^{-3}$ & 16.2 \\
$5.00 \times 10^{-5}$ & $1.50 \times 10^{-3}$ & $5.00 \times 10^{-3}$ & 22.6 \\
$5.00 \times 10^{-5}$ & $2.00 \times 10^{-3}$ & $5.00 \times 10^{-3}$ & 29.8 \\
\hline
\end{tabular}

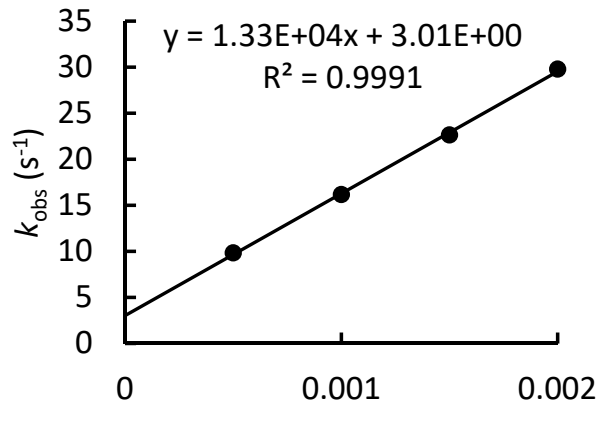

[1c] (M)

$$
k_{2}=1.33 \times 10^{4} \mathrm{M}^{-1} \mathrm{~s}^{-1}
$$

Table S15. Quinone methide $\mathbf{2 b}$ and sodium thiophenolate (Na-1c) in DMSO (detection at $486 \mathrm{~nm}$ )

\begin{tabular}{llll}
\hline$[\mathbf{2 b}]_{0}, \mathrm{M}$ & {$[\mathbf{1 c}]_{0}, \mathrm{M}$} & {$[\mathbf{1 c}-\mathrm{H}]_{0}, \mathrm{M}$} & $k_{\mathrm{obs}, \mathrm{s}^{-1}}$ \\
\hline $5.00 \times 10^{-5}$ & $5.00 \times 10^{-4}$ & $5.00 \times 10^{-3}$ & 17.0 \\
$5.00 \times 10^{-5}$ & $1.00 \times 10^{-3}$ & $5.00 \times 10^{-3}$ & 30.3 \\
$5.00 \times 10^{-5}$ & $1.50 \times 10^{-3}$ & $5.00 \times 10^{-3}$ & 44.1 \\
$5.00 \times 10^{-5}$ & $2.00 \times 10^{-3}$ & $5.00 \times 10^{-3}$ & 58.3 \\
\hline
\end{tabular}

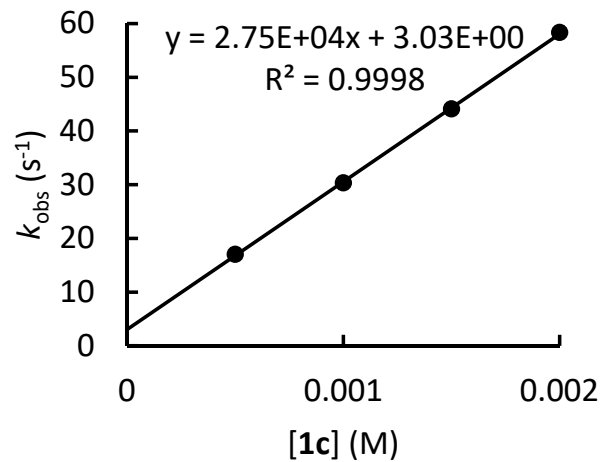

$$
k_{2}=2.75 \times 10^{4} \mathrm{M}^{-1} \mathrm{~s}^{-1}
$$

Table S16. Quinone methide $\mathbf{2 c}$ and sodium thiophenolate (Na-1c) in DMSO (detection at $393 \mathrm{~nm}$ )

\begin{tabular}{llll}
\hline$[\mathbf{2 c}]_{0}, \mathrm{M}$ & {$[\mathbf{1 c}]_{0}, \mathrm{M}$} & {$[\mathbf{1 c}-\mathrm{H}]_{0}, \mathrm{M}$} & $k_{\text {obs }}, \mathrm{s}^{-1}$ \\
\hline $5.00 \times 10^{-5}$ & $5.00 \times 10^{-4}$ & $5.00 \times 10^{-3}$ & 147 \\
$5.00 \times 10^{-5}$ & $1.00 \times 10^{-3}$ & $5.00 \times 10^{-3}$ & 256 \\
$5.00 \times 10^{-5}$ & $1.50 \times 10^{-3}$ & $5.00 \times 10^{-3}$ & 375 \\
$5.00 \times 10^{-5}$ & $2.00 \times 10^{-3}$ & $5.00 \times 10^{-3}$ & 483 \\
\hline
\end{tabular}

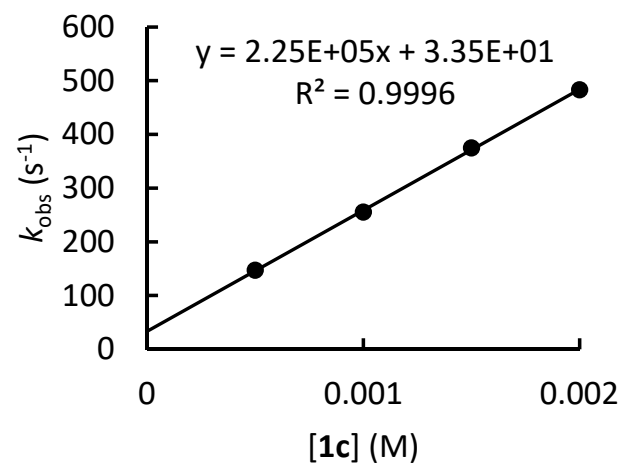

$k_{2}=2.25 \times 10^{5} \mathrm{M}^{-1} \mathrm{~s}^{-1}$ 
Table S17. Quinone methide $\mathbf{2 d}$ and potassium thiophenolate (K-1c) in DMSO (detection at $371 \mathrm{~nm}$ )

\begin{tabular}{llll}
\hline$[\mathbf{2 d}]_{0}, \mathrm{M}$ & {$[\mathbf{1 c}]_{0}, \mathrm{M}$} & {$[\mathbf{1 c}-\mathrm{H}]_{0}, \mathrm{M}$} & $k_{\mathrm{obs}}, \mathrm{s}^{-1}$ \\
\hline $5.00 \times 10^{-5}$ & $1.58 \times 10^{-4}$ & $5.00 \times 10^{-3}$ & 78.7 \\
$5.00 \times 10^{-5}$ & $3.16 \times 10^{-4}$ & $5.00 \times 10^{-3}$ & 152 \\
$5.00 \times 10^{-5}$ & $4.75 \times 10^{-4}$ & $5.00 \times 10^{-3}$ & 221 \\
$5.00 \times 10^{-5}$ & $6.33 \times 10^{-4}$ & $5.00 \times 10^{-3}$ & 299 \\
\hline
\end{tabular}

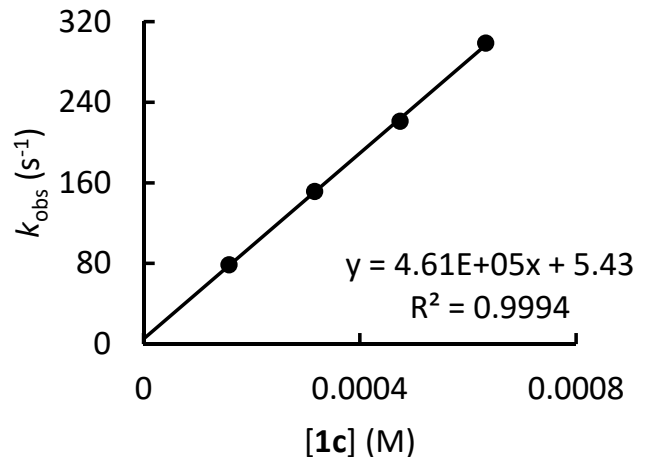

$k_{2}=4.61 \times 10^{5} \mathrm{M}^{-1} \mathrm{~s}^{-1}$

Table S18. Determination of $N$ and $s_{N}$ parameters for thiophenolate (1c) in DMSO.

\begin{tabular}{llll}
\hline $\begin{array}{l}\text { Quinone } \\
\text { methide }\end{array}$ & $E$ & $k_{2}, \mathrm{M}^{-1} \mathrm{~s}^{-1}$ & $\log k_{2}$ \\
\hline 2a & -17.90 & $1.33 \times 10^{4}$ & 4.12 \\
2b & -17.29 & $2.75 \times 10^{4}$ & 4.44 \\
2c & -16.11 & $2.25 \times 10^{5}$ & 5.35 \\
2d & -15.83 & $4.61 \times 10^{5}$ & 5.66 \\
\hline
\end{tabular}

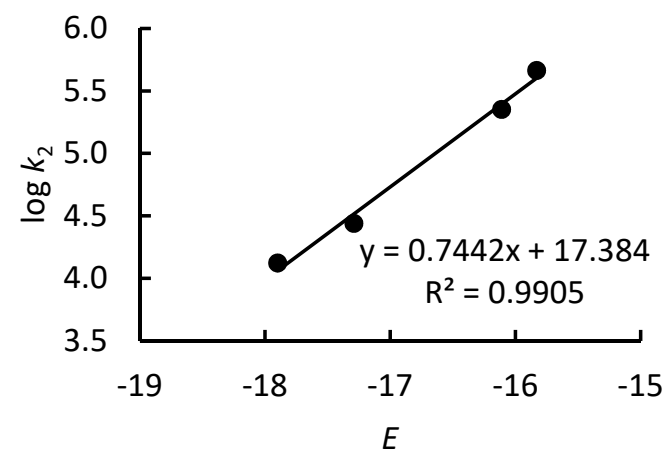

$N=23.36$

$s_{N}=0.74$ 
Kinetics of reactions of 4-bromo-thiophenolate (1d) with quinone methides 2

Table S19. Quinone methide 2a and potassium 4-bromo-thiophenolate (K-1d) in DMSO (detection at $521 \mathrm{~nm}$ )

\begin{tabular}{llll}
\hline$[\mathbf{2 a}]_{0}, \mathrm{M}$ & {$[\mathbf{1 d}]_{0}, \mathrm{M}$} & {$[\mathbf{1 d}-\mathrm{H}]_{0}, \mathrm{M}$} & $k_{\text {obs }}, \mathrm{s}^{-1}$ \\
\hline $5.00 \times 10^{-5}$ & $3.70 \times 10^{-4}$ & $5.00 \times 10^{-3}$ & 2.91 \\
$5.00 \times 10^{-5}$ & $7.40 \times 10^{-4}$ & $5.00 \times 10^{-3}$ & 5.70 \\
$5.00 \times 10^{-5}$ & $1.11 \times 10^{-3}$ & $5.00 \times 10^{-3}$ & 8.87 \\
$5.00 \times 10^{-5}$ & $1.48 \times 10^{-3}$ & $5.00 \times 10^{-3}$ & 11.8 \\
\hline
\end{tabular}

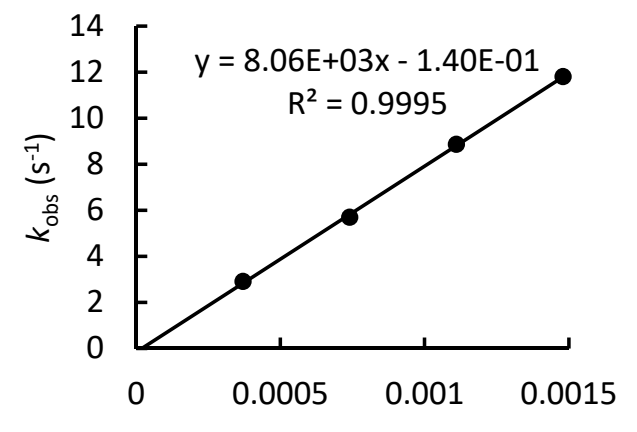

[1d] (M)

$$
k_{2}=8.06 \times 10^{3} \mathrm{M}^{-1} \mathrm{~s}^{-1}
$$

Table S20. Quinone methide $\mathbf{2 b}$ and potassium 4-bromo-thiophenolate (K-1d) in DMSO (detection at $490 \mathrm{~nm}$ )

\begin{tabular}{llll}
\hline$[\mathbf{2 b}]_{0}, \mathrm{M}$ & {$[\mathbf{1 d}]_{0}, \mathrm{M}$} & {$[\mathbf{1 d}-\mathrm{H}]_{0}, \mathrm{M}$} & $k_{\text {obs }, \mathrm{s}^{-1}}$ \\
\hline $5.00 \times 10^{-5}$ & $3.70 \times 10^{-4}$ & $5.00 \times 10^{-3}$ & 6.46 \\
$5.00 \times 10^{-5}$ & $7.40 \times 10^{-4}$ & $5.00 \times 10^{-3}$ & 13.0 \\
$5.00 \times 10^{-5}$ & $1.11 \times 10^{-3}$ & $5.00 \times 10^{-3}$ & 20.7 \\
$5.00 \times 10^{-5}$ & $1.48 \times 10^{-3}$ & $5.00 \times 10^{-3}$ & 26.6 \\
\hline
\end{tabular}

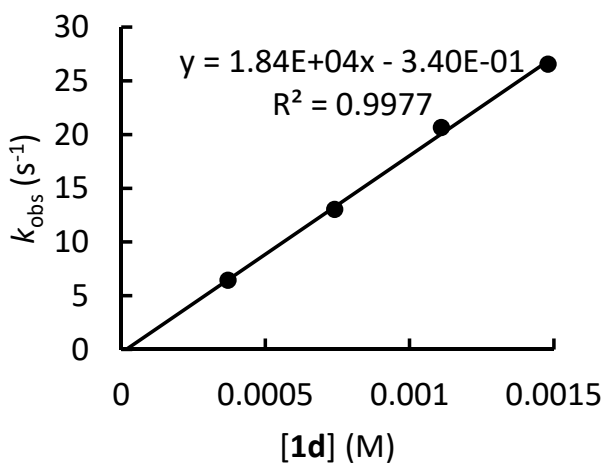

$$
k_{2}=1.84 \times 10^{4} \mathrm{M}^{-1} \mathrm{~s}^{-1}
$$

Table S21. Quinone methide $\mathbf{2 c}$ and potassium 4-bromo-thiophenolate (K-1d) in DMSO (detection at $390 \mathrm{~nm}$ )

\begin{tabular}{llll}
\hline$[\mathbf{2 c}]_{0}, \mathrm{M}$ & {$[\mathbf{1 d}]_{0}, \mathrm{M}$} & {$[\mathbf{1 d}-\mathrm{H}]_{0}, \mathrm{M}$} & $k_{\text {obs }, \mathrm{s}^{-1}}$ \\
\hline $5.00 \times 10^{-5}$ & $3.70 \times 10^{-4}$ & $5.00 \times 10^{-3}$ & 55.3 \\
$5.00 \times 10^{-5}$ & $7.40 \times 10^{-4}$ & $5.00 \times 10^{-3}$ & 113 \\
$5.00 \times 10^{-5}$ & $1.11 \times 10^{-3}$ & $5.00 \times 10^{-3}$ & 170 \\
$5.00 \times 10^{-5}$ & $1.48 \times 10^{-3}$ & $5.00 \times 10^{-3}$ & 220 \\
\hline
\end{tabular}

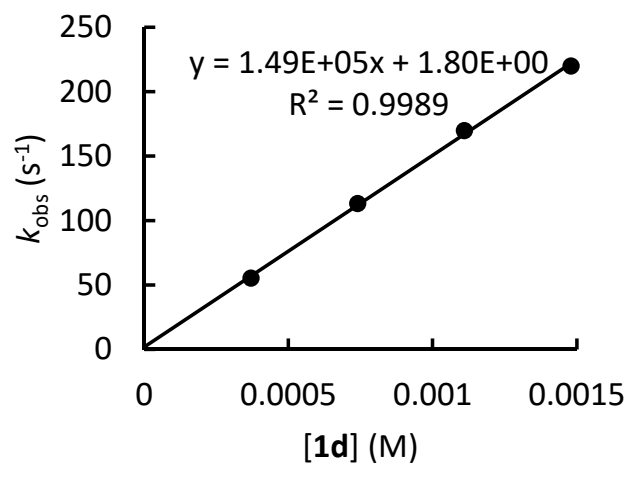

$k_{2}=1.49 \times 10^{5} \mathrm{M}^{-1} \mathrm{~s}^{-1}$ 
Table S22. Quinone methide 2d and potassium 4-bromo-thiophenolate (K-1d) in DMSO (detection at $371 \mathrm{~nm}$ )

\begin{tabular}{llll}
\hline$[\mathbf{2 d}]_{0}, \mathrm{M}$ & {$[\mathbf{1 d}]_{0}, \mathrm{M}$} & {$[\mathbf{1 d}-\mathrm{H}]_{0}, \mathrm{M}$} & $k_{\text {obs }}, \mathrm{s}^{-1}$ \\
\hline $5.00 \times 10^{-5}$ & $3.70 \times 10^{-4}$ & $5.00 \times 10^{-3}$ & 92.6 \\
$5.00 \times 10^{-5}$ & $7.40 \times 10^{-4}$ & $5.00 \times 10^{-3}$ & 191 \\
$5.00 \times 10^{-5}$ & $1.11 \times 10^{-3}$ & $5.00 \times 10^{-3}$ & 304 \\
$5.00 \times 10^{-5}$ & $1.48 \times 10^{-3}$ & $5.00 \times 10^{-3}$ & 404 \\
\hline
\end{tabular}

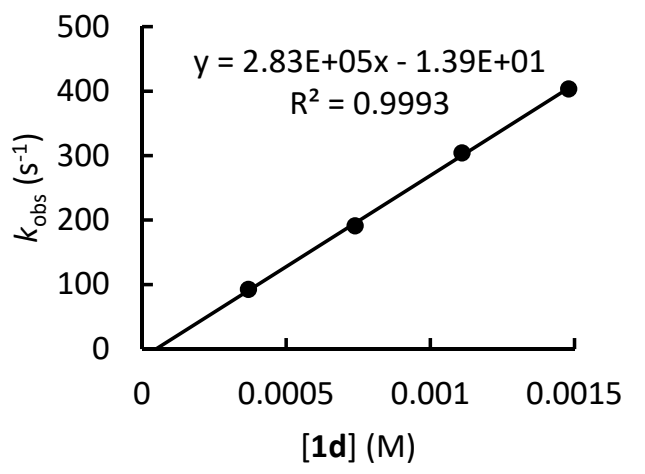

$$
k_{2}=2.83 \times 10^{5} \mathrm{M}^{-1} \mathrm{~s}^{-1}
$$

Table S23. Quinone methide $\mathbf{2 e}$ and potassium 4-bromo-thiophenolate (K-1d) in DMSO (detection at $350 \mathrm{~nm}$ )

\begin{tabular}{llll}
\hline$[\mathbf{2 e}]_{0}, \mathrm{M}$ & {$[\mathbf{1 d}]_{0}, \mathrm{M}$} & {$[\mathbf{1 d}-\mathrm{H}]_{0}, \mathrm{M}$} & $k_{\text {obs }}, \mathrm{s}^{-1}$ \\
\hline $5.00 \times 10^{-5}$ & $1.85 \times 10^{-4}$ & $5.00 \times 10^{-3}$ & 225 \\
$5.00 \times 10^{-5}$ & $2.78 \times 10^{-4}$ & $5.00 \times 10^{-3}$ & 323 \\
$5.00 \times 10^{-5}$ & $3.70 \times 10^{-4}$ & $5.00 \times 10^{-3}$ & 505 \\
$5.00 \times 10^{-5}$ & $7.40 \times 10^{-4}$ & $5.00 \times 10^{-3}$ & $1.01 \times 10^{3}$ \\
$5.00 \times 10^{-5}$ & $1.11 \times 10^{-3}$ & $5.00 \times 10^{-3}$ & $1.50 \times 10^{3}$ \\
\hline
\end{tabular}

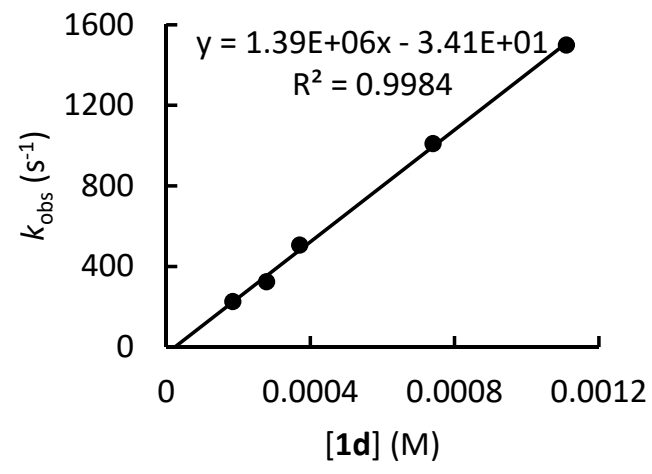

$$
k_{2}=1.39 \times 10^{6} \mathrm{M}^{-1} \mathrm{~s}^{-1}
$$

Table S24. Determination of $N$ and $s_{N}$ parameters for 4-bromo-thiophenolate (K-1d) in DMSO.

\begin{tabular}{llll}
\hline $\begin{array}{l}\text { Quinone } \\
\text { methide }\end{array}$ & $E$ & $k_{2}, \mathrm{M}^{-1} \mathrm{~s}^{-1}$ & $\log k_{2}$ \\
\hline $\mathbf{2 a}$ & -17.90 & $8.06 \times 10^{3}$ & 3.91 \\
$\mathbf{2 b}$ & -17.29 & $1.84 \times 10^{4}$ & 4.26 \\
$\mathbf{2 c}$ & -16.11 & $1.49 \times 10^{5}$ & 5.17 \\
$\mathbf{2 d}$ & -15.83 & $2.83 \times 10^{5}$ & 5.45 \\
$\mathbf{2 e}$ & -15.03 & $1.39 \times 10^{6}$ & 6.14 \\
\hline
\end{tabular}

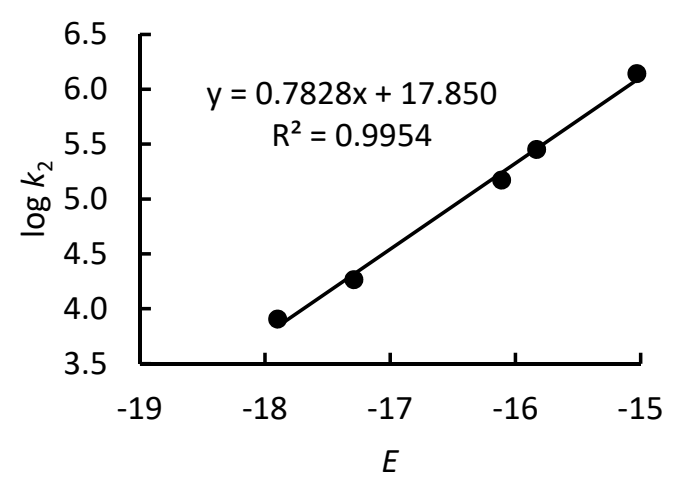

$N=22.80$

$S_{N}=0.78$ 
Kinetics of reactions of 3-chloro-thiophenolate (1e) with quinone methides 2

Table S25. Quinone methide $\mathbf{2 a}$ and potassium 3-chloro-thiophenolate (K-1e) in DMSO (detection at $520 \mathrm{~nm}$ )

\begin{tabular}{llll}
\hline$[2 \mathrm{a}]_{0}, \mathrm{M}$ & {$[\mathbf{1 e}]_{0}, \mathrm{M}$} & {$[1 \mathrm{e}-\mathrm{H}]_{0}, \mathrm{M}$} & $k_{\mathrm{obs}}, \mathrm{s}^{-1}$ \\
\hline $5.00 \times 10^{-5}$ & $2.04 \times 10^{-4}$ & $5.00 \times 10^{-3}$ & 1.03 \\
$5.00 \times 10^{-5}$ & $4.08 \times 10^{-4}$ & $5.00 \times 10^{-3}$ & 1.83 \\
$5.00 \times 10^{-5}$ & $6.12 \times 10^{-4}$ & $5.00 \times 10^{-3}$ & 2.69 \\
$5.00 \times 10^{-5}$ & $8.16 \times 10^{-4}$ & $5.00 \times 10^{-3}$ & 3.72 \\
\hline
\end{tabular}

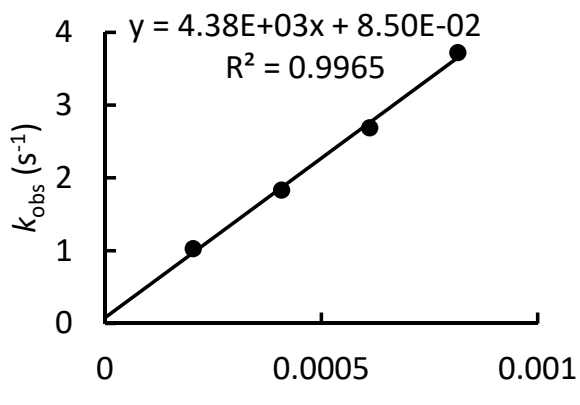

[1e] (M)

$$
k_{2}=4.38 \times 10^{3} \mathrm{M}^{-1} \mathrm{~s}^{-1}
$$

Table S26. Quinone methide $\mathbf{2 b}$ and potassium 3-chloro-thiophenolate (K-1e) in DMSO (detection at $490 \mathrm{~nm}$ )

\begin{tabular}{llll}
\hline$[\mathbf{2 b}]_{0}, \mathrm{M}$ & {$[\mathbf{1 e}]_{0}, \mathrm{M}$} & {$[\mathbf{1 e}-\mathrm{H}]_{0}, \mathrm{M}$} & $k_{\text {obs }}, \mathrm{s}^{-1}$ \\
\hline $5.00 \times 10^{-5}$ & $2.04 \times 10^{-4}$ & $5.00 \times 10^{-3}$ & 2.06 \\
$5.00 \times 10^{-5}$ & $4.08 \times 10^{-4}$ & $5.00 \times 10^{-3}$ & 3.82 \\
$5.00 \times 10^{-5}$ & $6.12 \times 10^{-4}$ & $5.00 \times 10^{-3}$ & 5.84 \\
$5.00 \times 10^{-5}$ & $8.16 \times 10^{-4}$ & $5.00 \times 10^{-3}$ & 8.21 \\
\hline
\end{tabular}

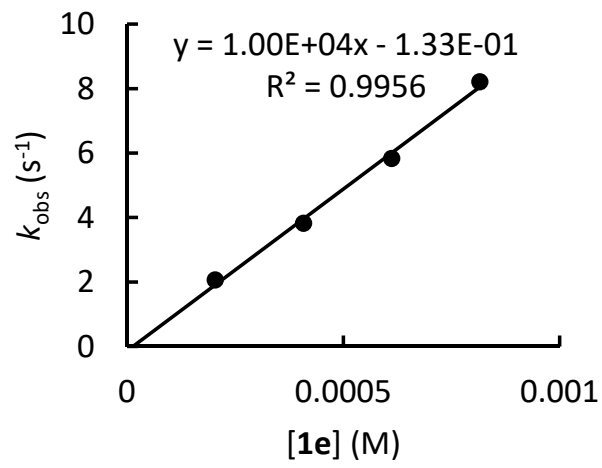

$$
k_{2}=1.00 \times 10^{4} \mathrm{M}^{-1} \mathrm{~s}^{-1}
$$

Table S27. Quinone methide $\mathbf{2 c}$ and potassium 3-chloro-thiophenolate (K-1e) in DMSO (detection at $393 \mathrm{~nm}$ )

\begin{tabular}{llll}
\hline$[\mathbf{2 c}]_{0}, \mathrm{M}$ & {$[\mathbf{1 e}]_{0}, \mathrm{M}$} & {$[\mathbf{1 e}-\mathrm{H}]_{0}, \mathrm{M}$} & $k_{\text {obs }, \mathrm{s}^{-1}}$ \\
\hline $5.00 \times 10^{-5}$ & $2.04 \times 10^{-4}$ & $5.00 \times 10^{-3}$ & 18.9 \\
$5.00 \times 10^{-5}$ & $4.08 \times 10^{-4}$ & $5.00 \times 10^{-3}$ & 35.4 \\
$5.00 \times 10^{-5}$ & $6.12 \times 10^{-4}$ & $5.00 \times 10^{-3}$ & 52.1 \\
$5.00 \times 10^{-5}$ & $8.16 \times 10^{-4}$ & $5.00 \times 10^{-3}$ & 75.8 \\
\hline
\end{tabular}

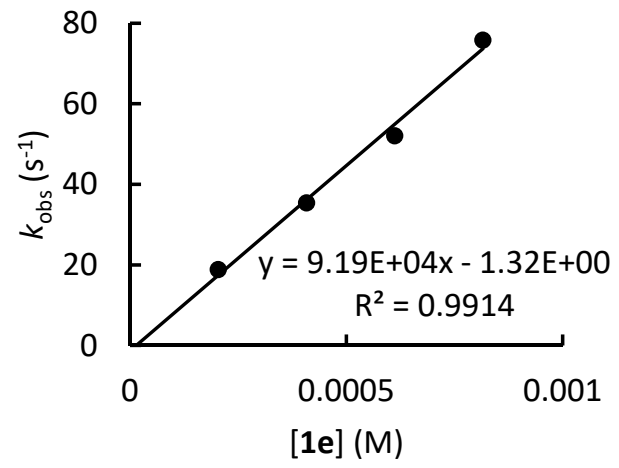

$k_{2}=9.19 \times 10^{4} \mathrm{M}^{-1} \mathrm{~s}^{-1}$ 
Table S28. Quinone methide 2d and sodium 3-chloro-thiophenolate (Na-1e) in DMSO (detection at $371 \mathrm{~nm}$ )

\begin{tabular}{llll}
\hline$[\mathbf{2 d}]_{0}, \mathrm{M}$ & {$[\mathbf{1 e}]_{0}, \mathrm{M}$} & {$[\mathbf{1 e}-\mathrm{H}]_{0}, \mathrm{M}$} & $k_{\mathrm{obs}}, \mathrm{s}^{-1}$ \\
\hline $5.00 \times 10^{-5}$ & $5.00 \times 10^{-4}$ & $5.00 \times 10^{-3}$ & 68.1 \\
$5.00 \times 10^{-5}$ & $1.00 \times 10^{-3}$ & $5.00 \times 10^{-3}$ & 124 \\
$5.00 \times 10^{-5}$ & $1.50 \times 10^{-3}$ & $5.00 \times 10^{-3}$ & 187 \\
$5.00 \times 10^{-5}$ & $2.00 \times 10^{-3}$ & $5.00 \times 10^{-3}$ & 237 \\
\hline
\end{tabular}

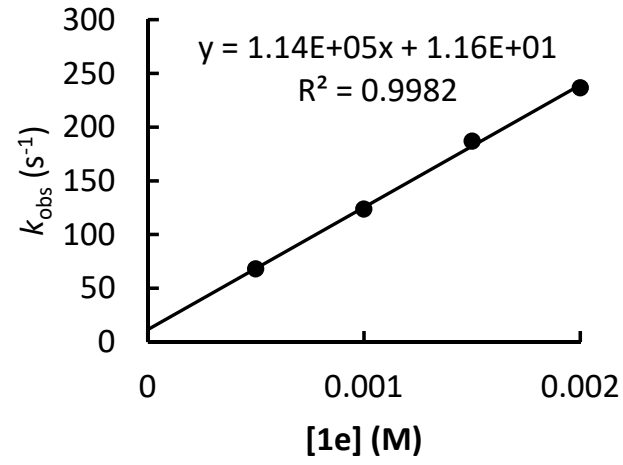

$$
k_{2}=1.14 \times 10^{5} \mathrm{M}^{-1} \mathrm{~s}^{-1}
$$

Table S29. Quinone methide $\mathbf{2 e}$ and potassium 3-chloro-thiophenolate (K-1e) in DMSO (detection at $350 \mathrm{~nm}$ )

\begin{tabular}{llll}
\hline$[\mathbf{2 e}]_{0}, \mathrm{M}$ & {$[\mathbf{1 e}]_{0}, \mathrm{M}$} & {$[\mathbf{1 e}-\mathrm{H}]_{0}, \mathrm{M}$} & $k_{\text {obs }}, \mathrm{s}^{-1}$ \\
\hline $5.00 \times 10^{-5}$ & $2.04 \times 10^{-4}$ & $5.00 \times 10^{-3}$ & 156 \\
$5.00 \times 10^{-5}$ & $4.08 \times 10^{-4}$ & $5.00 \times 10^{-3}$ & 298 \\
$5.00 \times 10^{-5}$ & $6.12 \times 10^{-4}$ & $5.00 \times 10^{-3}$ & 466 \\
$5.00 \times 10^{-5}$ & $8.16 \times 10^{-4}$ & $5.00 \times 10^{-3}$ & 640 \\
\hline
\end{tabular}

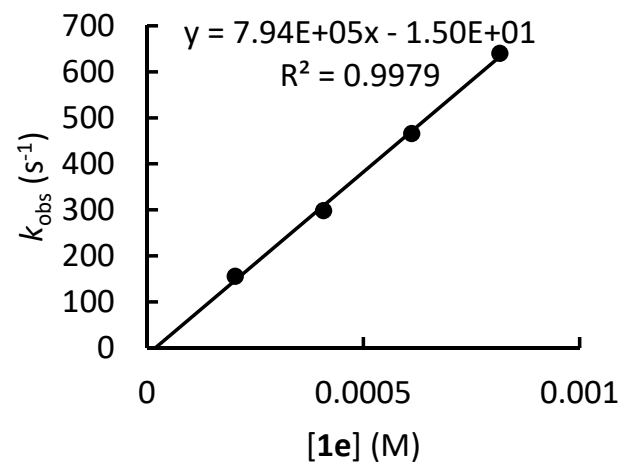

$$
k_{2}=7.94 \times 10^{5} \mathrm{M}^{-1} \mathrm{~s}^{-1}
$$

Table S30. Determination of $N$ and $s_{N}$ parameters for 3-chloro-thiophenolate (1e) in DMSO.

\begin{tabular}{llll}
\hline $\begin{array}{l}\text { Quinone } \\
\text { methide }\end{array}$ & $E$ & $k_{2}, \mathrm{M}^{-1} \mathrm{~s}^{-1}$ & $\log k_{2}$ \\
\hline $\mathbf{2 a}$ & -17.90 & $4.38 \times 10^{3}$ & 3.64 \\
$\mathbf{2 b}$ & -17.29 & $1.00 \times 10^{4}$ & 4.00 \\
$\mathbf{2 c}$ & -16.11 & $9.19 \times 10^{4}$ & 4.96 \\
$\mathbf{2 d}$ & -15.83 & $1.14 \times 10^{5}$ & 5.06 \\
$\mathbf{2 e}$ & -15.03 & $7.94 \times 10^{6}$ & 5.90 \\
\hline
\end{tabular}

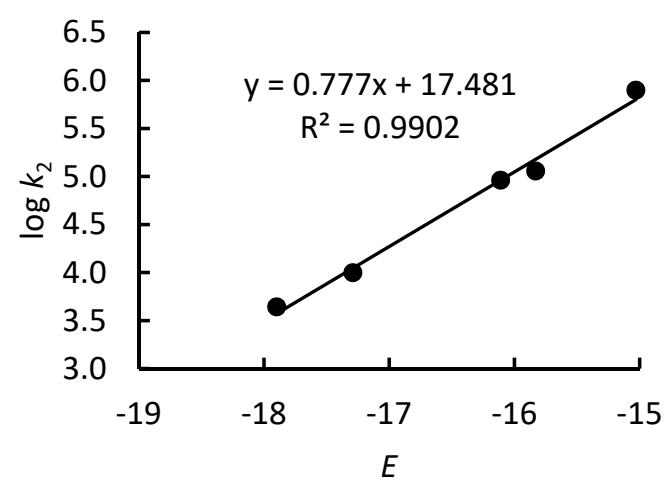

$N=22.50$

$s_{N}=0.78$ 


\section{Kinetics of reactions of 3-(trifluoromethyl)thiophenolate (1f) with quinone methides 2}

Table S31. Quinone methide 2a and potassium 3-(trifluoromethyl)thiophenolate (K-1f) in DMSO (detection at $520 \mathrm{~nm}$ )

\begin{tabular}{llll}
\hline$[\mathbf{2 a}]_{0}, \mathrm{M}$ & {$[\mathbf{1 f}]_{0}, \mathrm{M}$} & {$[\mathbf{1 f}-\mathrm{H}]_{0}, \mathrm{M}$} & $k_{\mathrm{obs}, \mathrm{s}^{-1}}$ \\
\hline $5.00 \times 10^{-5}$ & $4.01 \times 10^{-4}$ & $5.00 \times 10^{-3}$ & 1.23 \\
$5.00 \times 10^{-5}$ & $8.02 \times 10^{-4}$ & $5.00 \times 10^{-3}$ & 2.09 \\
$5.00 \times 10^{-5}$ & $1.20 \times 10^{-3}$ & $5.00 \times 10^{-3}$ & 3.04 \\
$5.00 \times 10^{-5}$ & $1.60 \times 10^{-3}$ & $5.00 \times 10^{-3}$ & 4.02 \\
\hline
\end{tabular}

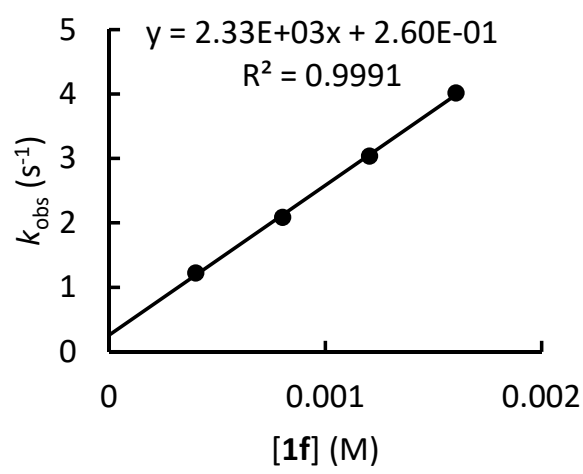

$$
k_{2}=2.33 \times 10^{3} \mathrm{M}^{-1} \mathrm{~s}^{-1}
$$

Table S32. Quinone methide 2b and potassium 3-(trifluoromethyl)thiophenolate (K-1f) in DMSO (detection at $490 \mathrm{~nm})$

\begin{tabular}{llll}
\hline$[\mathbf{2 b}]_{0}, \mathrm{M}$ & {$[\mathbf{1 f}]_{0}, \mathrm{M}$} & {$[\mathbf{1 f}-\mathrm{H}]_{0}, \mathrm{M}$} & $k_{\text {obs }}, \mathrm{s}^{-1}$ \\
\hline $5.00 \times 10^{-5}$ & $4.01 \times 10^{-4}$ & $5.00 \times 10^{-3}$ & 2.36 \\
$5.00 \times 10^{-5}$ & $8.02 \times 10^{-4}$ & $5.00 \times 10^{-3}$ & 4.45 \\
$5.00 \times 10^{-5}$ & $1.20 \times 10^{-3}$ & $5.00 \times 10^{-3}$ & 6.81 \\
$5.00 \times 10^{-5}$ & $1.60 \times 10^{-3}$ & $5.00 \times 10^{-3}$ & 9.10 \\
\hline
\end{tabular}

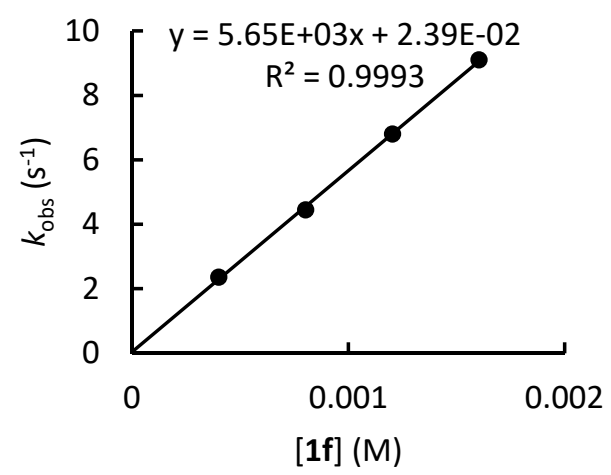

$$
k_{2}=5.65 \times 10^{3} \mathrm{M}^{-1} \mathrm{~s}^{-1}
$$

Table S33. Quinone methide $\mathbf{2 c}$ and potassium 3-(trifluoromethyl)thiophenolate (K-1f) in DMSO (detection at $393 \mathrm{~nm})$

\begin{tabular}{llll}
\hline$[\mathbf{2 c}]_{0}, \mathrm{M}$ & {$[\mathbf{1 f}]_{0}, \mathrm{M}$} & {$[\mathbf{1 f}-\mathrm{H}]_{0}, \mathrm{M}$} & $k_{\mathrm{obs},} \mathrm{s}^{-1}$ \\
\hline $5.00 \times 10^{-5}$ & $4.01 \times 10^{-4}$ & $5.00 \times 10^{-3}$ & 22.7 \\
$5.00 \times 10^{-5}$ & $8.02 \times 10^{-4}$ & $5.00 \times 10^{-3}$ & 43.8 \\
$5.00 \times 10^{-5}$ & $1.20 \times 10^{-3}$ & $5.00 \times 10^{-3}$ & 68.0 \\
$5.00 \times 10^{-5}$ & $1.60 \times 10^{-3}$ & $5.00 \times 10^{-3}$ & 89.6 \\
\hline
\end{tabular}

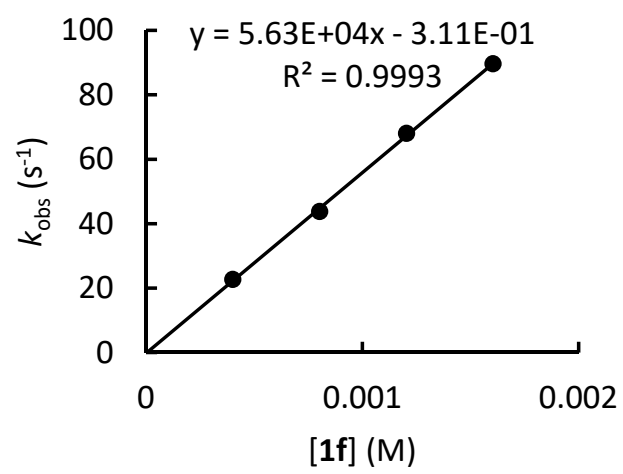

$k_{2}=5.63 \times 10^{4} \mathrm{M}^{-1} \mathrm{~s}^{-1}$ 
Table S34. Quinone methide 2e and potassium 3-(trifluoromethyl)thiophenolate (K-1f) in DMSO (detection at $350 \mathrm{~nm}$ )

\begin{tabular}{llll}
\hline$[\mathbf{2 e}]_{0}, \mathrm{M}$ & {$[\mathbf{1 f}]_{0}, \mathrm{M}$} & {$[\mathbf{1 f}-\mathrm{H}]_{0}, \mathrm{M}$} & $k_{\text {obs }}, \mathrm{s}^{-1}$ \\
\hline $5.00 \times 10^{-5}$ & $2.01 \times 10^{-4}$ & $5.00 \times 10^{-3}$ & 104 \\
$5.00 \times 10^{-5}$ & $4.01 \times 10^{-4}$ & $5.00 \times 10^{-3}$ & 198 \\
$5.00 \times 10^{-5}$ & $8.02 \times 10^{-3}$ & $5.00 \times 10^{-3}$ & 447 \\
$5.00 \times 10^{-5}$ & $1.20 \times 10^{-3}$ & $5.00 \times 10^{-3}$ & 740 \\
\hline
\end{tabular}

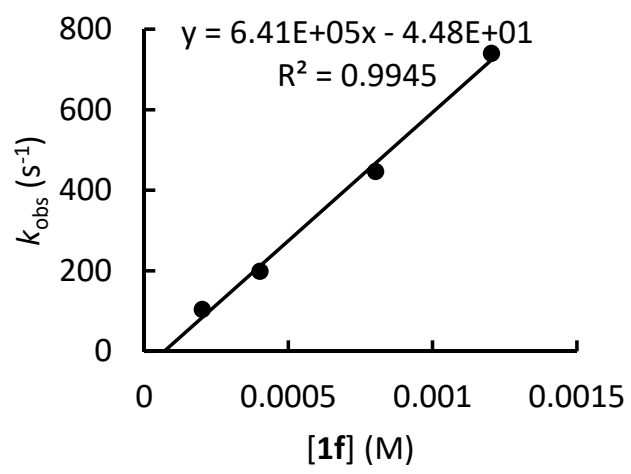

$k_{2}=6.41 \times 10^{5} \mathrm{M}^{-1} \mathrm{~s}^{-1}$

Table S35. Determination of $N$ and $s_{N}$ parameters for 3-(trifluoromethyl)thiophenolate (1f) in DMSO.

\begin{tabular}{llll}
\hline $\begin{array}{l}\text { Quinone } \\
\text { methide }\end{array}$ & $E$ & $k_{2}, \mathrm{M}^{-1} \mathrm{~s}^{-1}$ & $\log k_{2}$ \\
\hline $\mathbf{2 a}$ & -17.90 & $2.33 \times 10^{3}$ & 3.37 \\
$\mathbf{2 b}$ & -17.29 & $5.65 \times 10^{3}$ & 3.75 \\
$\mathbf{2 c}$ & -16.11 & $5.63 \times 10^{4}$ & 4.75 \\
$\mathbf{2 e}$ & -15.03 & $6.41 \times 10^{5}$ & 5.80 \\
\hline
\end{tabular}

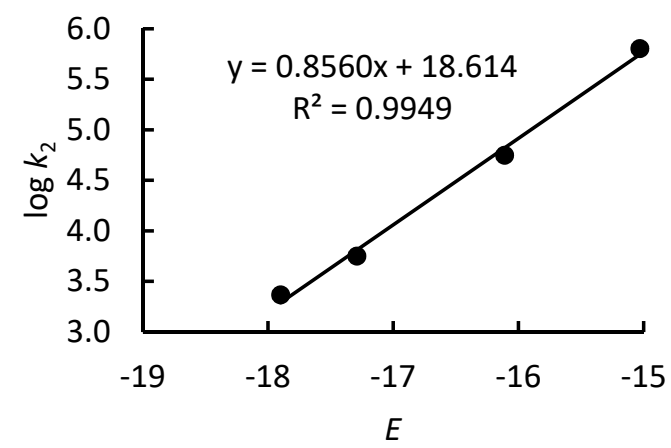

$N=21.75$

$s_{N}=0.86$ 


\section{Kinetics of reactions of 4-(trifluoromethyl)thiophenolate $(1 \mathrm{~g})$ with quinone methides 2}

Table S36. Quinone methide $\mathbf{2 a}$ and potassium or sodium 4-(trifluoromethyl)thiophenolate (K/Na-1g) in DMSO (detection at $520 \mathrm{~nm}$ )

\begin{tabular}{llll}
\hline$[\mathbf{2 a}]_{0}, \mathrm{M}$ & {$[\mathbf{1 g}]_{0}, \mathrm{M}$} & {$[\mathbf{1 g}-\mathrm{H}]_{0}, \mathrm{M}$} & $k_{\mathrm{obs}, \mathrm{s}^{-1}}$ \\
\hline $5.00 \times 10^{-5}$ & $4.91 \times 10^{-4}$ & $5.00 \times 10^{-3}$ & 0.912 \\
$5.00 \times 10^{-5}$ & $9.82 \times 10^{-4}$ & $5.00 \times 10^{-3}$ & 1.42 \\
$5.00 \times 10^{-5}$ & $1.03 \times 10^{-3 a}$ & $5.00 \times 10^{-3}$ & 1.41 \\
$5.00 \times 10^{-5}$ & $1.47 \times 10^{-3}$ & $5.00 \times 10^{-3}$ & 1.79 \\
$5.00 \times 10^{-5}$ & $1.96 \times 10^{-3}$ & $5.00 \times 10^{-3}$ & 2.15 \\
$5.00 \times 10^{-5}$ & $2.05 \times 10^{-3 a}$ & $5.00 \times 10^{-3}$ & 2.21 \\
\hline
\end{tabular}

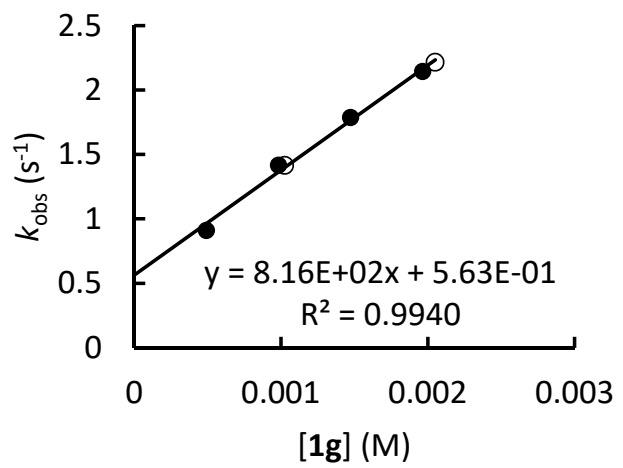

${ }^{a}$ The counterion $\mathrm{Na}^{+}$was used instead of $\mathrm{K}^{+}$.

$$
k_{2}=8.16 \times 10^{2} \mathrm{M}^{-1} \mathrm{~s}^{-1}
$$

Table S37. Quinone methide $\mathbf{2 b}$ and potassium or sodium 4-(trifluoromethyl)thiophenolate (K/Na-1g) in DMSO (detection at $490 \mathrm{~nm}$ )

\begin{tabular}{llll}
\hline$[\mathbf{2 b}]_{0}, \mathrm{M}$ & {$[\mathbf{1 g}-\mathrm{K}]_{0}, \mathrm{M}$} & {$[\mathbf{1 g - H}]_{0}, \mathrm{M}$} & $k_{\text {obs, }} \mathrm{s}^{-1}$ \\
\hline $5.00 \times 10^{-5}$ & $4.91 \times 10^{-4}$ & $5.00 \times 10^{-3}$ & 1.51 \\
$5.00 \times 10^{-5}$ & $9.82 \times 10^{-4}$ & $5.00 \times 10^{-3}$ & 3.01 \\
$5.00 \times 10^{-5}$ & $1.03 \times 10^{-3} a$ & $5.00 \times 10^{-3}$ & 3.01 \\
$5.00 \times 10^{-5}$ & $1.47 \times 10^{-3}$ & $5.00 \times 10^{-3}$ & 4.22 \\
$5.00 \times 10^{-5}$ & $1.96 \times 10^{-3}$ & $5.00 \times 10^{-3}$ & 5.39 \\
\hline
\end{tabular}

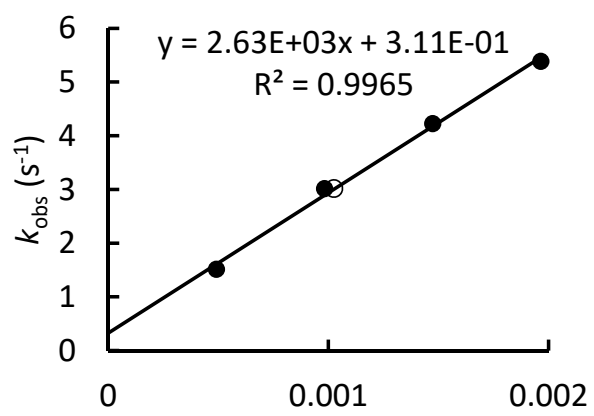

[1g] (M)

${ }^{a}$ The counterion $\mathrm{Na}^{+}$was used instead of $\mathrm{K}^{+}$.

$$
k_{2}=2.63 \times 10^{3} \mathrm{M}^{-1} \mathrm{~s}^{-1}
$$

Table S38. Quinone methide $\mathbf{2 c}$ and potassium 4-(trifluoromethyl)thiophenolate (K-1g) in DMSO (detection at $393 \mathrm{~nm})$

\begin{tabular}{llll}
\hline$[\mathbf{2 c}]_{0}, \mathrm{M}$ & {$[\mathbf{1 g}]_{0}, \mathrm{M}$} & {$[\mathbf{1 g}-\mathrm{H}]_{0}, \mathrm{M}$} & $k_{\text {obs }}, \mathrm{s}^{-1}$ \\
\hline $5.00 \times 10^{-5}$ & $4.91 \times 10^{-4}$ & $5.00 \times 10^{-3}$ & 15.2 \\
$5.00 \times 10^{-5}$ & $9.82 \times 10^{-4}$ & $5.00 \times 10^{-3}$ & 30.8 \\
$5.00 \times 10^{-5}$ & $1.47 \times 10^{-3}$ & $5.00 \times 10^{-3}$ & 43.6 \\
$5.00 \times 10^{-5}$ & $1.96 \times 10^{-3}$ & $5.00 \times 10^{-3}$ & 57.5 \\
\hline
\end{tabular}

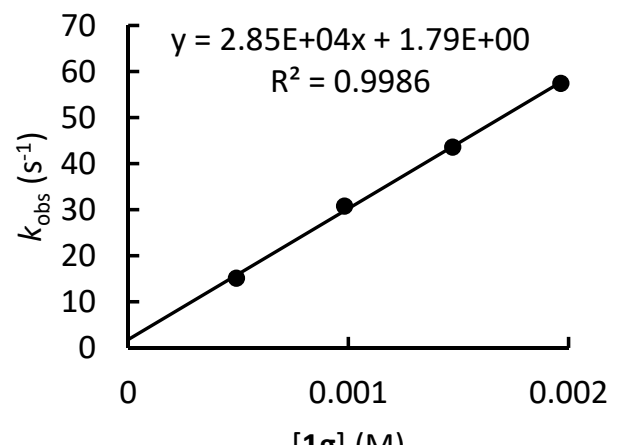

[1g] (M)

$k_{2}=2.85 \times 10^{4} \mathrm{M}^{-1} \mathrm{~s}^{-1}$ 
Table S39. Quinone methide 2c and sodium 4-(trifluoromethyl)thiophenolate (Na-1g) in DMSO (detection at $393 \mathrm{~nm})$

\begin{tabular}{llll}
\hline$[\mathbf{2 c}]_{0}, \mathrm{M}$ & {$[\mathbf{1 g}]_{0}, \mathrm{M}$} & {$[\mathbf{1 g}-\mathrm{H}]_{0}, \mathrm{M}$} & $k_{\mathrm{obs}, \mathrm{s}^{-1}}$ \\
\hline $5.00 \times 10^{-5}$ & $5.00 \times 10^{-4}$ & $5.00 \times 10^{-3}$ & 15.8 \\
$5.00 \times 10^{-5}$ & $1.00 \times 10^{-3}$ & $5.00 \times 10^{-3}$ & 28.8 \\
$5.00 \times 10^{-5}$ & $1.50 \times 10^{-3}$ & $5.00 \times 10^{-3}$ & 40.8 \\
$5.00 \times 10^{-5}$ & $2.00 \times 10^{-3}$ & $5.00 \times 10^{-3}$ & 57.7 \\
\hline
\end{tabular}

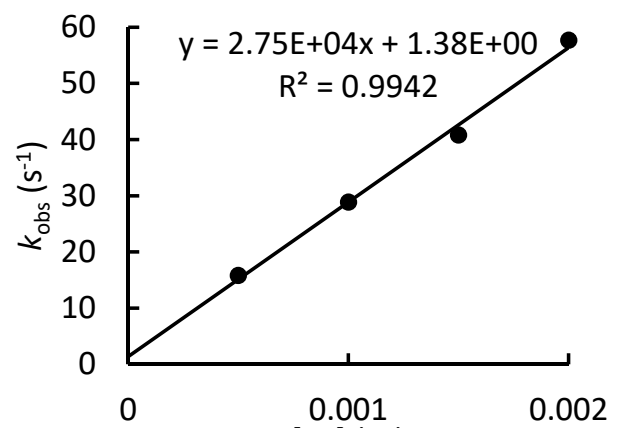

$$
k_{2}=2.75 \times 10^{4} \mathrm{M}^{-1} \mathrm{~s}^{-1}
$$

[1g] (M)

Table S40. Quinone methide $\mathbf{2 d}$ and sodium 4-(trifluoromethyl)thiophenolate (Na-1g) in DMSO (detection at 371 $\mathrm{nm})$

\begin{tabular}{llll}
\hline$[\mathbf{2 d}]_{0}, \mathrm{M}$ & {$[\mathbf{1 g}]_{0}, \mathrm{M}$} & {$[\mathbf{1 g - H}]_{0}, \mathrm{M}$} & $k_{\text {obs }, \mathrm{s}^{-1}}$ \\
\hline $5.00 \times 10^{-5}$ & $5.00 \times 10^{-4}$ & $5.00 \times 10^{-3}$ & 26.6 \\
$5.00 \times 10^{-5}$ & $1.00 \times 10^{-3}$ & $5.00 \times 10^{-3}$ & 52.9 \\
$5.00 \times 10^{-5}$ & $1.50 \times 10^{-3}$ & $5.00 \times 10^{-3}$ & 78.7 \\
$5.00 \times 10^{-5}$ & $2.00 \times 10^{-3}$ & $5.00 \times 10^{-3}$ & 109 \\
\hline
\end{tabular}

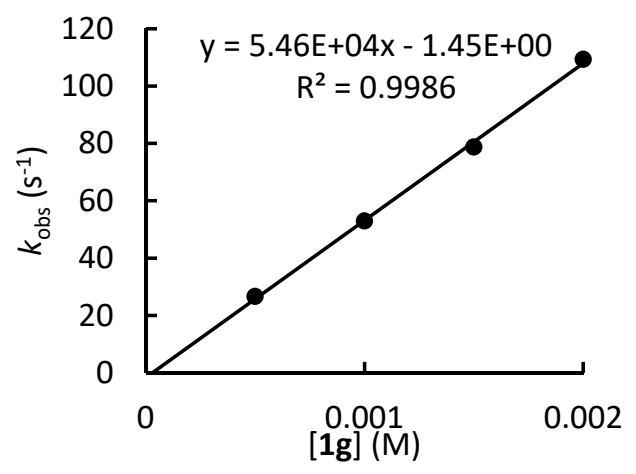

$$
k_{2}=5.46 \times 10^{4} \mathrm{M}^{-1} \mathrm{~s}^{-1}
$$

Table S41. Quinone methide $\mathbf{2 e}$ and sodium 4-(trifluoromethyl)thiophenolate (Na-1g) in DMSO (detection at 350 $\mathrm{nm}$ )

\begin{tabular}{llll}
\hline$[\mathbf{2 e}]_{0}, \mathrm{M}$ & {$[\mathbf{1 g}]_{0}, \mathrm{M}$} & {$[\mathbf{1 g - H}]_{0}, \mathrm{M}$} & $k_{\mathrm{obs}, \mathrm{s}^{-1}}$ \\
\hline $5.00 \times 10^{-5}$ & $5.00 \times 10^{-4}$ & $5.00 \times 10^{-3}$ & 134 \\
$5.00 \times 10^{-5}$ & $1.00 \times 10^{-3}$ & $5.00 \times 10^{-3}$ & 270 \\
$5.00 \times 10^{-5}$ & $1.50 \times 10^{-3}$ & $5.00 \times 10^{-3}$ & 408 \\
$5.00 \times 10^{-5}$ & $2.00 \times 10^{-3}$ & $5.00 \times 10^{-3}$ & 551 \\
\hline
\end{tabular}

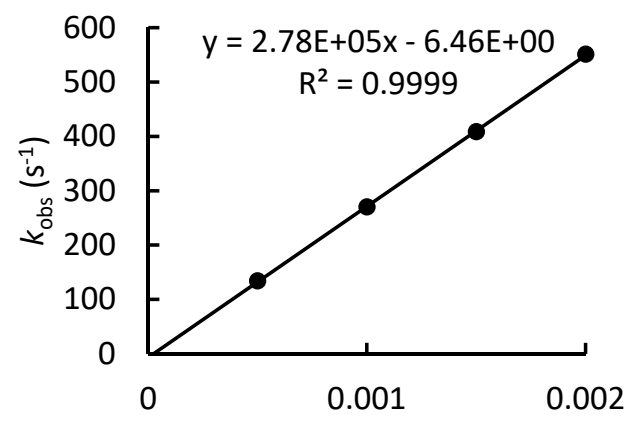

[1g] (M)

$k_{2}=2.78 \times 10^{5} \mathrm{M}^{-1} \mathrm{~s}^{-1}$ 
Table S42. Quinone methide $\mathbf{2 f}$ and sodium 4-(trifluoromethyl)thiophenolate (Na-1g) in DMSO (detection at $374 \mathrm{~nm}$ )

\begin{tabular}{llll}
\hline$[\mathbf{2 f}]_{0}, \mathrm{M}$ & {$[\mathbf{1 g}]_{0}, \mathrm{M}$} & {$[\mathbf{1 g}-\mathrm{H}]_{0}, \mathrm{M}$} & $k_{\text {obs }}, \mathrm{s}^{-1}$ \\
\hline $5.00 \times 10^{-5}$ & $5.00 \times 10^{-4}$ & $5.00 \times 10^{-3}$ & 627 \\
$5.00 \times 10^{-5}$ & $1.00 \times 10^{-3}$ & $5.00 \times 10^{-3}$ & $1.07 \times 10^{3}$ \\
$5.00 \times 10^{-5}$ & $1.50 \times 10^{-3}$ & $5.00 \times 10^{-3}$ & $1.40 \times 10^{3}$ \\
$5.00 \times 10^{-5}$ & $2.00 \times 10^{-3}$ & $5.00 \times 10^{-3}$ & $1.81 \times 10^{3}$ \\
\hline
\end{tabular}

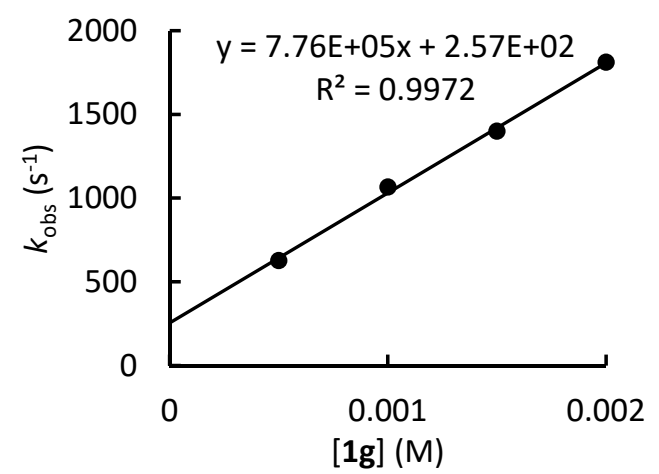

$$
k_{2}=7.76 \times 10^{5} \mathrm{M}^{-1} \mathrm{~s}^{-1}
$$

Table S43. Determination of $N$ and $s_{N}$ parameters for 4-(trifluoromethyl)thiophenolate (1g) in DMSO.

\begin{tabular}{llll}
\hline $\begin{array}{l}\text { Quinone } \\
\text { methide }\end{array}$ & $E$ & $k_{2}, \mathrm{M}^{-1} \mathrm{~s}^{-1}$ & $\log k_{2}$ \\
\hline $\mathbf{2 a}$ & -17.90 & $8.16 \times 10^{2}$ & 2.91 \\
$\mathbf{2 b}$ & -17.29 & $2.63 \times 10^{3}$ & 3.42 \\
$\mathbf{2 c}$ & -16.11 & $2.75 \times 10^{4}$ & 4.45 \\
$\mathbf{2 d}$ & -15.83 & $5.46 \times 10^{4}$ & 4.74 \\
$\mathbf{2 e}$ & -15.03 & $2.78 \times 10^{5}$ & 5.44 \\
$\mathbf{2 f}$ & -14.36 & $7.76 \times 10^{5}$ & 5.89 \\
\hline
\end{tabular}

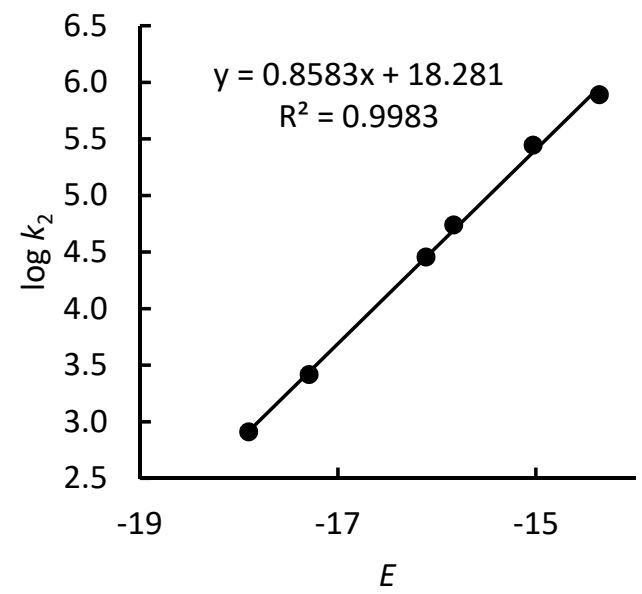

$N=21.30$

$s_{N}=0.86$ 


\section{Kinetics of reactions of 3,5-bis(trifluoromethyl)thiophenolate (1h) with quinone methides 2}

Table S44. Quinone methide $\mathbf{2 c}$ and potassium 3,5-bis(trifluoromethyl)thiophenolate (K-1h) in DMSO (detection at $394 \mathrm{~nm}$ )

\begin{tabular}{llll}
\hline$[\mathbf{2 c}]_{0}, \mathrm{M}$ & {$[\mathbf{1} \mathbf{h}]_{0}, \mathrm{M}$} & {$[\mathbf{1 h}-\mathrm{H}]_{0}, \mathrm{M}$} & $k_{\mathrm{obs}}, \mathrm{s}^{-1}$ \\
\hline $5.00 \times 10^{-5}$ & $1.27 \times 10^{-4}$ & $5.00 \times 10^{-3}$ & 0.208 \\
$5.00 \times 10^{-5}$ & $2.53 \times 10^{-4}$ & $5.00 \times 10^{-3}$ & 0.358 \\
$5.00 \times 10^{-5}$ & $5.06 \times 10^{-4}$ & $5.00 \times 10^{-3}$ & 0.661 \\
$5.00 \times 10^{-5}$ & $1.01 \times 10^{-3}$ & $5.00 \times 10^{-3}$ & 1.25 \\
\hline
\end{tabular}

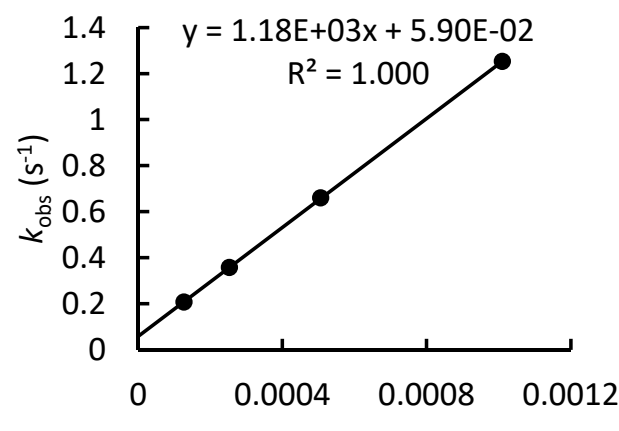

[1h] (M)

$$
k_{2}=1.18 \times 10^{3} \mathrm{M}^{-1} \mathrm{~s}^{-1}
$$

Table S45. Quinone methide $\mathbf{2 d}$ and potassium 3,5-bis(trifluoromethyl)thiophenolate (K-1h) in DMSO (detection at $372 \mathrm{~nm}$ )

\begin{tabular}{llll}
\hline$[\mathbf{2 d}]_{0}, \mathrm{M}$ & {$[\mathbf{1 h}]_{0}, \mathrm{M}$} & {$[\mathbf{1 h}-\mathrm{H}]_{0}, \mathrm{M}$} & $k_{\mathrm{obs}}, \mathrm{s}^{-1}$ \\
\hline $5.00 \times 10^{-5}$ & $1.27 \times 10^{-4}$ & $5.00 \times 10^{-3}$ & 0.432 \\
$5.00 \times 10^{-5}$ & $2.53 \times 10^{-4}$ & $5.00 \times 10^{-3}$ & 0.724 \\
$5.00 \times 10^{-5}$ & $5.06 \times 10^{-4}$ & $5.00 \times 10^{-3}$ & 1.32 \\
$5.00 \times 10^{-5}$ & $1.01 \times 10^{-3}$ & $5.00 \times 10^{-3}$ & 2.50 \\
\hline
\end{tabular}

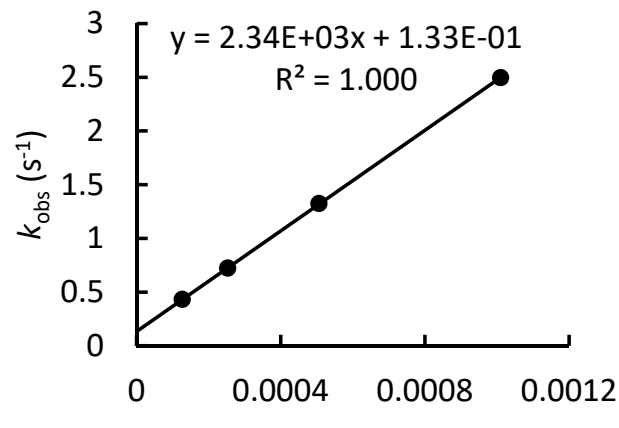

[1h] (M)

$$
k_{2}=2.34 \times 10^{3} \mathrm{M}^{-1} \mathrm{~s}^{-1}
$$

Table S46. Quinone methide $\mathbf{2 e}$ and potassium 3,5-bis(trifluoromethyl)thiophenolate (K-1h) in DMSO (detection at $386 \mathrm{~nm}$ )

\begin{tabular}{llll}
\hline$[\mathbf{2 e}]_{0}, \mathrm{M}$ & {$[\mathbf{1 h}]_{0}, \mathrm{M}$} & {$[\mathbf{1 h}-\mathrm{H}]_{0}, \mathrm{M}$} & $k_{\mathrm{obs}}, \mathrm{s}^{-1}$ \\
\hline $3.50 \times 10^{-4}$ & $1.27 \times 10^{-4}$ & $5.00 \times 10^{-3}$ & 2.12 \\
$3.50 \times 10^{-4}$ & $2.53 \times 10^{-4}$ & $5.00 \times 10^{-3}$ & 3.82 \\
$3.50 \times 10^{-4}$ & $5.06 \times 10^{-4}$ & $5.00 \times 10^{-3}$ & 6.82 \\
$3.50 \times 10^{-4}$ & $1.01 \times 10^{-3}$ & $5.00 \times 10^{-3}$ & 13.4 \\
\hline
\end{tabular}

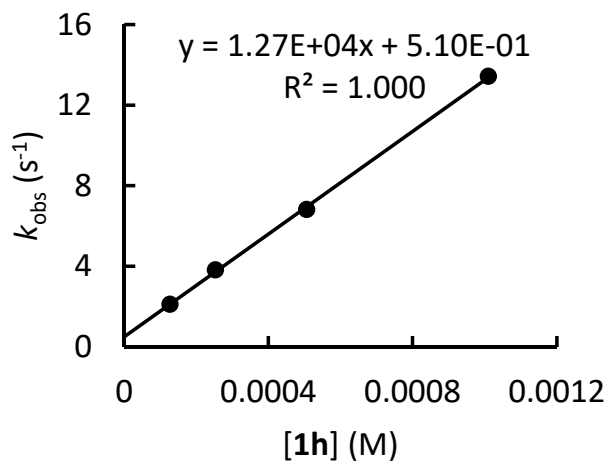

$k_{2}=1.27 \times 10^{4} \mathrm{M}^{-1} \mathrm{~s}^{-1}$ 
Table S47. Quinone methide $\mathbf{2 f}$ and potassium 3,5-bis(trifluoromethyl)thiophenolate (K-1h) in DMSO (detection at $374 \mathrm{~nm}$ )

\begin{tabular}{llll}
\hline$[\mathbf{2 f}]_{0}, \mathrm{M}$ & {$[\mathbf{1 h}]_{0}, \mathrm{M}$} & {$[\mathbf{1 h}-\mathrm{H}]_{0}, \mathrm{M}$} & $k_{\mathrm{obs}, \mathrm{s}^{-1}}$ \\
\hline $5.00 \times 10^{-5}$ & $1.27 \times 10^{-4}$ & $5.00 \times 10^{-3}$ & 6.43 \\
$5.00 \times 10^{-5}$ & $2.53 \times 10^{-4}$ & $5.00 \times 10^{-3}$ & 11.2 \\
$5.00 \times 10^{-5}$ & $5.06 \times 10^{-4}$ & $5.00 \times 10^{-3}$ & 21.0 \\
$5.00 \times 10^{-5}$ & $1.01 \times 10^{-3}$ & $5.00 \times 10^{-3}$ & 40.0 \\
\hline
\end{tabular}

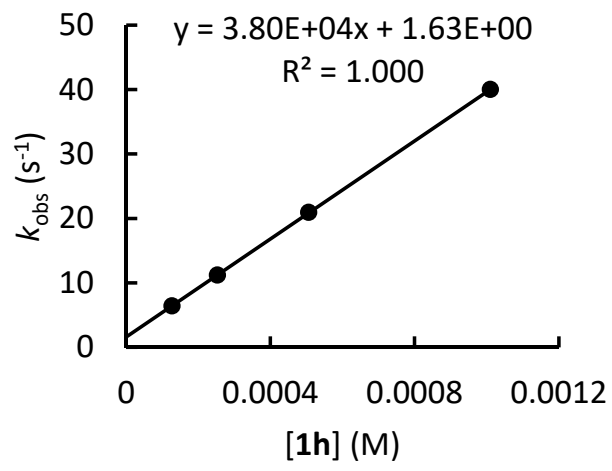

$k_{2}=3.80 \times 10^{4} \mathrm{M}^{-1} \mathrm{~s}^{-1}$

Table S48. Determination of $N$ and $s_{N}$ parameters for 3,5-bis(trifluoromethyl)thiophenolate (1h) in DMSO.

\begin{tabular}{llll}
\hline $\begin{array}{l}\text { Quinone } \\
\text { methide }\end{array}$ & $E$ & $k_{2}, \mathrm{M}^{-1} \mathrm{~s}^{-1}$ & $\log k_{2}$ \\
\hline $\mathbf{2 c}$ & -16.11 & $1.18 \times 10^{3}$ & 3.07 \\
$\mathbf{2 d}$ & -15.83 & $2.34 \times 10^{3}$ & 3.37 \\
$\mathbf{2 e}$ & -15.03 & $1.27 \times 10^{4}$ & 4.11 \\
$\mathbf{2 f}$ & -14.36 & $3.80 \times 10^{4}$ & 4.58 \\
\hline
\end{tabular}

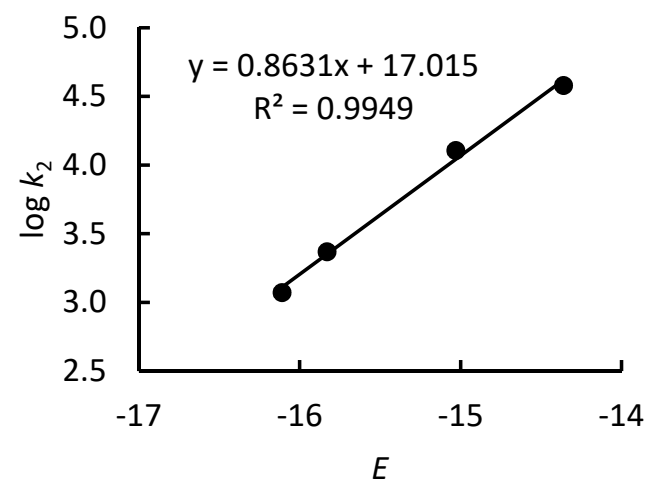

$N=19.71$

$s_{N}=0.86$ 


\section{Kinetics of reactions of 4-nitro-thiophenolate (1i) with quinone methides}

Table S49. Quinone methide $2 \mathrm{c}$ and sodium 4-nitro-thiophenolate (Na-1i) in DMSO (detection at $392 \mathrm{~nm}$ )

\begin{tabular}{llll}
\hline$[\mathbf{2 c}]_{0}, \mathrm{M}$ & {$[\mathbf{1 i}]_{0}, \mathrm{M}$} & {$[\mathbf{1 h}-\mathrm{H}]_{0}, \mathrm{M}$} & $k_{\text {obs }}, \mathrm{s}^{-1}$ \\
\hline $6.20 \times 10^{-5}$ & $1.59 \times 10^{-4}$ & $5.00 \times 10^{-3}$ & $9.54 \times 10^{-2}$ \\
$6.20 \times 10^{-5}$ & $3.13 \times 10^{-4}$ & $5.00 \times 10^{-3}$ & $1.39 \times 10^{-1}$ \\
$6.20 \times 10^{-5}$ & $4.61 \times 10^{-4}$ & $5.00 \times 10^{-3}$ & $1.74 \times 10^{-1}$ \\
$6.20 \times 10^{-5}$ & $6.45 \times 10^{-4}$ & $5.00 \times 10^{-3}$ & $2.19 \times 10^{-1}$ \\
\hline
\end{tabular}

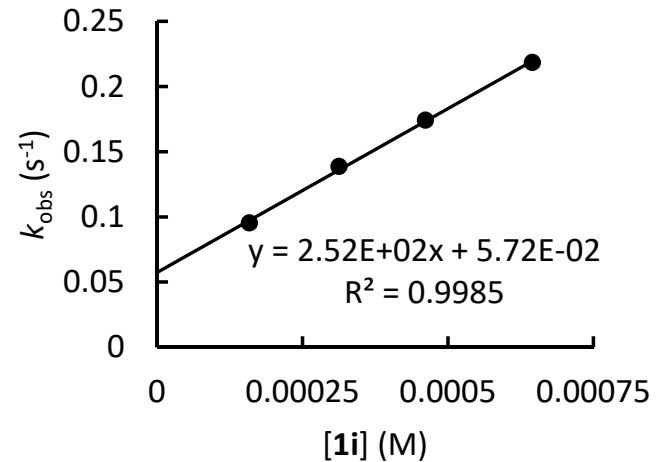

$k_{2}=2.52 \times 10^{2} \mathrm{M}^{-1} \mathrm{~s}^{-1}$

Table S50. Quinone methide $\mathbf{2 d}$ and sodium 4-nitro-thiophenolate ( $\mathrm{Na}-\mathbf{1 i}$ ) in DMSO (detection at $390 \mathrm{~nm}$ )

\begin{tabular}{llll}
\hline$[\mathbf{2 d}]_{0}, \mathrm{M}$ & {$[\mathbf{1 i}]_{0}, \mathrm{M}$} & {$[\mathbf{1 h}-\mathrm{H}]_{0}, \mathrm{M}$} & $k_{\text {obs }}, \mathrm{s}^{-1}$ \\
\hline $1.12 \times 10^{-4}$ & $1.59 \times 10^{-4}$ & $5.00 \times 10^{-3}$ & $1.91 \times 10^{-1}$ \\
$1.12 \times 10^{-4}$ & $3.13 \times 10^{-4}$ & $5.00 \times 10^{-3}$ & $2.76 \times 10^{-1}$ \\
$1.12 \times 10^{-4}$ & $4.61 \times 10^{-4}$ & $5.00 \times 10^{-3}$ & $3.50 \times 10^{-1}$ \\
$1.12 \times 10^{-4}$ & $6.45 \times 10^{-4}$ & $5.00 \times 10^{-3}$ & $4.42 \times 10^{-1}$ \\
\hline
\end{tabular}

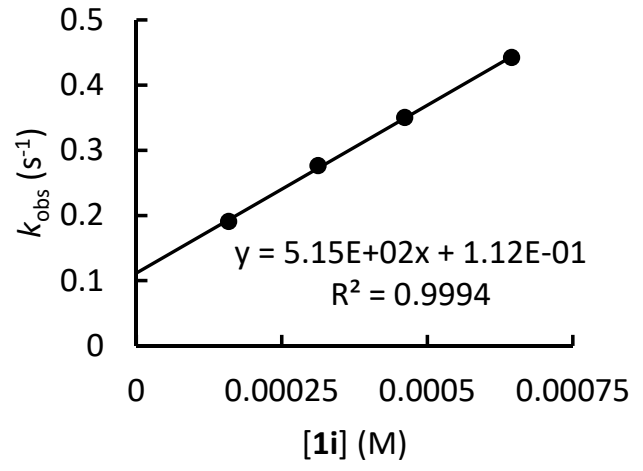

$k_{2}=5.15 \times 10^{2} \mathrm{M}^{-1} \mathrm{~s}^{-1}$

Table S51. Quinone methide $\mathbf{2 e}$ and sodium 4-nitro-thiophenolate ( $\mathrm{Na}-\mathbf{1 i}$ ) in DMSO (detection at $390 \mathrm{~nm}$ )

\begin{tabular}{llll}
\hline$[\mathbf{2 e}]_{0}, \mathrm{M}$ & {$[\mathbf{1 i}]_{0}, \mathrm{M}$} & {$[\mathbf{1 h}-\mathrm{H}]_{0}, \mathrm{M}$} & $k_{\text {obs }}, \mathrm{s}^{-1}$ \\
\hline $3.75 \times 10^{-4}$ & $1.59 \times 10^{-4}$ & $5.00 \times 10^{-3}$ & 0.930 \\
$3.75 \times 10^{-4}$ & $3.13 \times 10^{-4}$ & $5.00 \times 10^{-3}$ & 1.44 \\
$3.75 \times 10^{-4}$ & $4.61 \times 10^{-4}$ & $5.00 \times 10^{-3}$ & 1.83 \\
$3.75 \times 10^{-4}$ & $6.45 \times 10^{-4}$ & $5.00 \times 10^{-3}$ & 2.23 \\
\hline
\end{tabular}

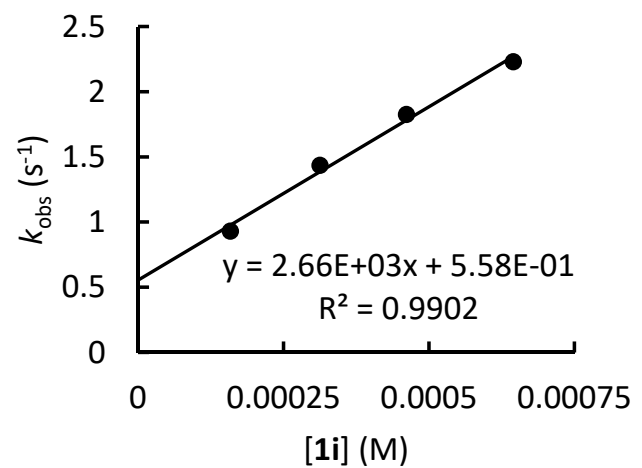

$k_{2}=2.66 \times 10^{3} \mathrm{M}^{-1} \mathrm{~s}^{-1}$ 
Table S52. Quinone methide $\mathbf{2 f}$ and sodium 4-nitro-thiophenolate (Na-1i) in DMSO (detection at $390 \mathrm{~nm}$ )

\begin{tabular}{llll}
\hline$[\mathbf{2 f}]_{0}, \mathrm{M}$ & {$[\mathbf{1 i}]_{0}, \mathrm{M}$} & {$[\mathbf{1 h}-\mathrm{H}]_{0}, \mathrm{M}$} & $k_{\mathrm{obs}}, \mathrm{s}^{-1}$ \\
\hline $1.12 \times 10^{-4}$ & $1.59 \times 10^{-4}$ & $5.00 \times 10^{-3}$ & 3.22 \\
$1.12 \times 10^{-4}$ & $3.13 \times 10^{-4}$ & $5.00 \times 10^{-3}$ & 4.95 \\
$1.12 \times 10^{-4}$ & $4.61 \times 10^{-4}$ & $5.00 \times 10^{-3}$ & 6.23 \\
$1.12 \times 10^{-4}$ & $6.45 \times 10^{-4}$ & $5.00 \times 10^{-3}$ & 7.37 \\
\hline
\end{tabular}

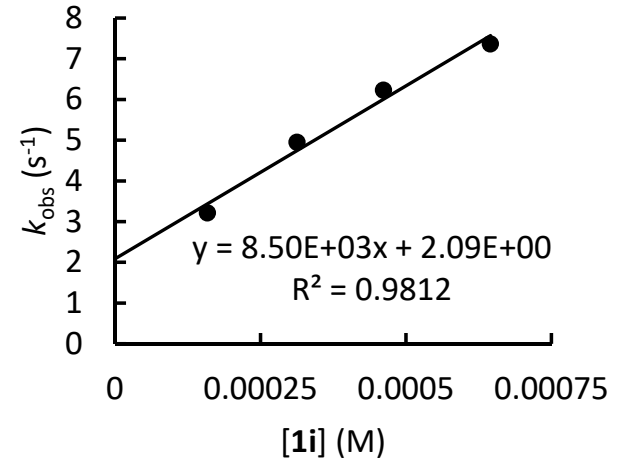

$$
k_{2}=8.50 \times 10^{3} \mathrm{M}^{-1} \mathrm{~s}^{-1}
$$

Table S53. Determination of $N$ and $s_{N}$ parameters for 4-nitro-thiophenolate (1i) in DMSO.

\begin{tabular}{llll}
\hline $\begin{array}{l}\text { Quinone } \\
\text { methide }\end{array}$ & $E$ & $k_{2}, \mathrm{M}^{-1} \mathrm{~s}^{-1}$ & $\log k_{2}$ \\
\hline $\mathbf{2 c}$ & -16.11 & $2.51 \times 10^{2}$ & 2.40 \\
$\mathbf{2 d}$ & -15.83 & $5.15 \times 10^{2}$ & 2.71 \\
$\mathbf{2 e}$ & -15.03 & $2.66 \times 10^{3}$ & 3.42 \\
$\mathbf{2 f}$ & -14.36 & $8.49 \times 10^{3}$ & 3.93 \\
\hline
\end{tabular}

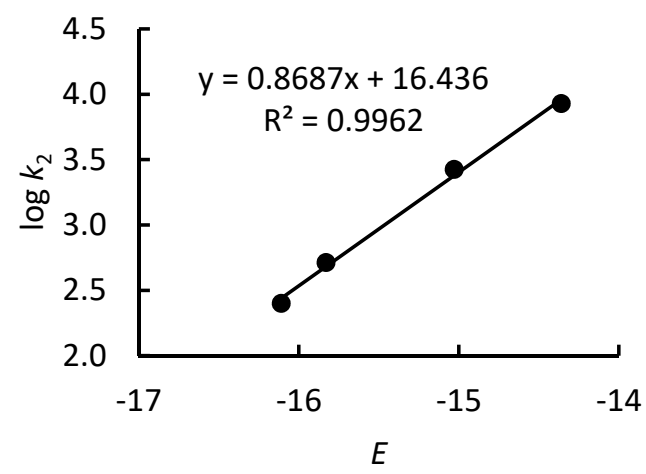

$N=18.91$

$S_{N}=0.87$ 
Kinetics of the reactions of napthalene-2-thiolate (1j) with quinone methides

Table S54. Quinone methide $\mathbf{2 b}$ and potassium napthalene-2-thiolate (K-1j) in DMSO (at $490 \mathrm{~nm}$ )

\begin{tabular}{llll}
\hline$[\mathbf{2 b}]_{0}, M$ & {$[\mathbf{1 j}]_{0}, M$} & {$[\mathbf{1 j}-\mathrm{H}]_{0}, \mathrm{M}$} & $k_{\text {obs }, \mathrm{s}^{-1}}$ \\
\hline $5.00 \times 10^{-5}$ & $9.92 \times 10^{-5}$ & $5.00 \times 10^{-3}$ & 2.70 \\
$5.00 \times 10^{-5}$ & $1.98 \times 10^{-4}$ & $5.00 \times 10^{-3}$ & 5.23 \\
$5.00 \times 10^{-5}$ & $2.97 \times 10^{-4}$ & $5.00 \times 10^{-3}$ & 8.30 \\
$5.00 \times 10^{-5}$ & $3.97 \times 10^{-4}$ & $5.00 \times 10^{-3}$ & 10.3 \\
\hline
\end{tabular}

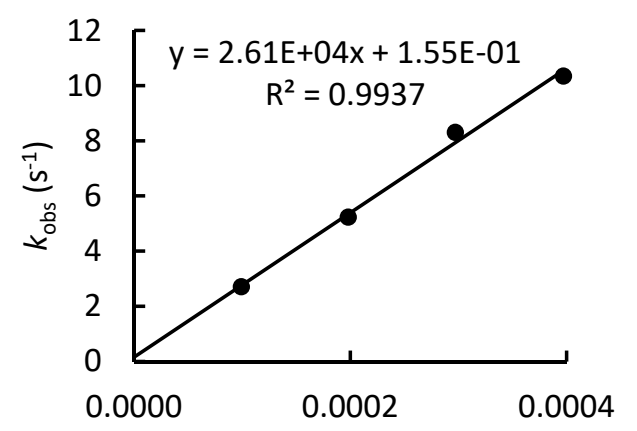

[1j] (M)

$$
k_{2}=2.61 \times 10^{4} \mathrm{M}^{-1} \mathrm{~s}^{-1}
$$

Table S55. Quinone methide $\mathbf{2 c}$ and potassium napthalene-2-thiolate (K-1j) in DMSO (detection at $393 \mathrm{~nm}$ )

\begin{tabular}{llll}
\hline$[\mathbf{2 c}]_{0}, \mathrm{M}$ & {$[\mathbf{1 j}]_{0}, \mathrm{M}$} & {$[\mathbf{1 j}-\mathrm{H}]_{0}, \mathrm{M}$} & $k_{\mathrm{obs}}, \mathrm{s}^{-1}$ \\
\hline $5.00 \times 10^{-5}$ & $9.92 \times 10^{-5}$ & $5.00 \times 10^{-3}$ & 21.9 \\
$5.00 \times 10^{-5}$ & $1.98 \times 10^{-4}$ & $5.00 \times 10^{-3}$ & 44.0 \\
$5.00 \times 10^{-5}$ & $2.97 \times 10^{-4}$ & $5.00 \times 10^{-3}$ & 62.9 \\
$5.00 \times 10^{-5}$ & $3.97 \times 10^{-4}$ & $5.00 \times 10^{-3}$ & 80.8 \\
\hline
\end{tabular}

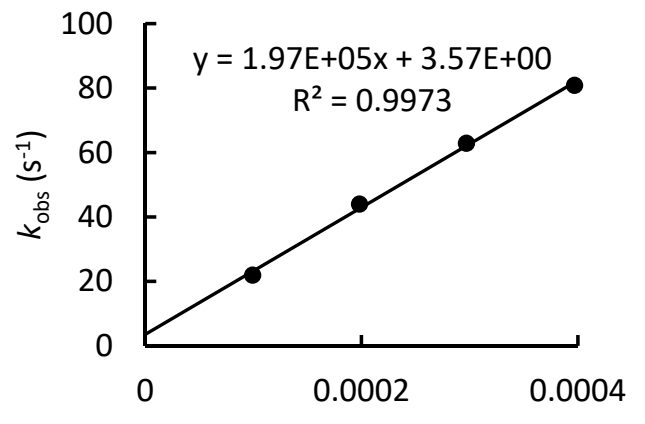

[1j] (M)

$$
k_{2}=1.97 \times 10^{5} \mathrm{M}^{-1} \mathrm{~s}^{-1}
$$

Table S56. Quinone methide $\mathbf{2} \mathbf{d}$ and potassium napthalene-2-thiolate (K-1j) in DMSO (detection at $371 \mathrm{~nm}$ )

\begin{tabular}{llll}
\hline$[\mathbf{2} \mathbf{d}]_{0}, M$ & {$[\mathbf{1} \mathbf{j}]_{0}, \mathrm{M}$} & {$[\mathbf{1} \mathbf{j}-\mathrm{H}]_{0}, \mathrm{M}$} & $k_{\mathrm{obs}}, \mathrm{s}^{-1}$ \\
\hline $5.00 \times 10^{-5}$ & $9.92 \times 10^{-5}$ & $5.00 \times 10^{-3}$ & 35.3 \\
$5.00 \times 10^{-5}$ & $1.98 \times 10^{-4}$ & $5.00 \times 10^{-3}$ & 76.5 \\
$5.00 \times 10^{-5}$ & $2.97 \times 10^{-4}$ & $5.00 \times 10^{-3}$ & 111 \\
$5.00 \times 10^{-5}$ & $3.97 \times 10^{-4}$ & $5.00 \times 10^{-3}$ & 139 \\
\hline
\end{tabular}

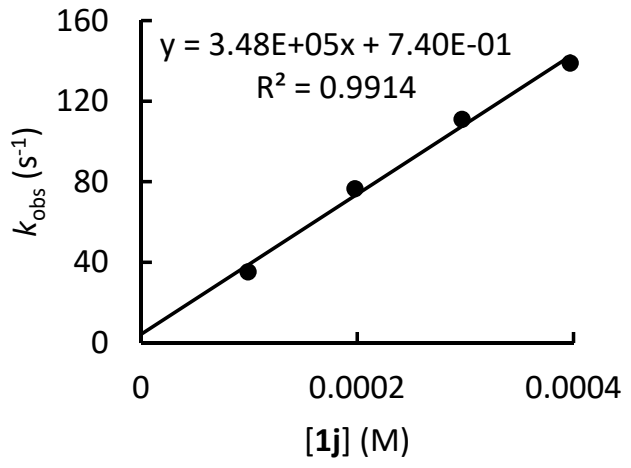

$k_{2}=3.48 \times 10^{5} \mathrm{M}^{-1} \mathrm{~s}^{-1}$ 
Table S57. Quinone methide $\mathbf{2 e}$ and potassium napthalene-2-thiolate (K-1j) in DMSO (detection at $350 \mathrm{~nm}$ )

\begin{tabular}{llll}
\hline$[\mathbf{2} \mathbf{e}]_{0}, \mathrm{M}$ & {$[\mathbf{1} \mathbf{j}]_{0}, \mathrm{M}$} & {$[\mathbf{1 j}-\mathrm{H}]_{0}, \mathrm{M}$} & $k_{\text {obs, }} \mathrm{s}^{-1}$ \\
\hline $5.00 \times 10^{-5}$ & $9.92 \times 10^{-5}$ & $5.00 \times 10^{-3}$ & 182 \\
$5.00 \times 10^{-5}$ & $1.98 \times 10^{-4}$ & $5.00 \times 10^{-3}$ & 431 \\
$5.00 \times 10^{-5}$ & $2.97 \times 10^{-4}$ & $5.00 \times 10^{-3}$ & 628 \\
$5.00 \times 10^{-5}$ & $3.97 \times 10^{-4}$ & $5.00 \times 10^{-3}$ & 795 \\
\hline
\end{tabular}

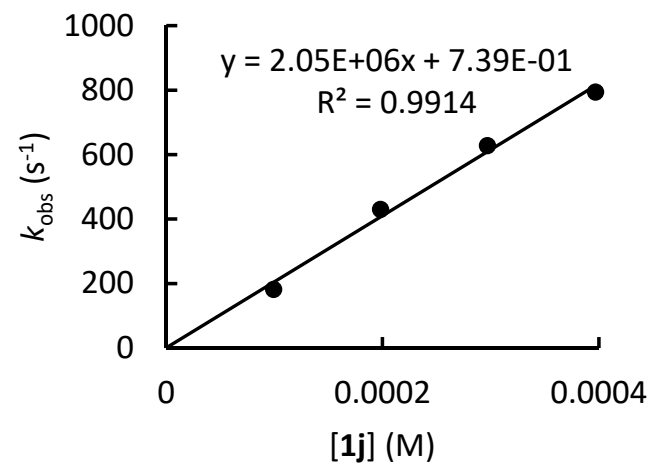

$k_{2}=2.05 \times 10^{6} \mathrm{M}^{-1} \mathrm{~s}^{-1}$

Table S58. Determination of $N$ and $s_{N}$ parameters for napthalene-2-thiolate (1j) in DMSO.

\begin{tabular}{llll}
\hline $\begin{array}{l}\text { Quinone } \\
\text { methide }\end{array}$ & $E$ & $k_{2}, \mathrm{M}^{-1} \mathrm{~s}^{-1}$ & $\log k_{2}$ \\
\hline $\mathbf{2 b}$ & -17.29 & $2.61 \times 10^{4}$ & 4.42 \\
$\mathbf{2 c}$ & -16.11 & $1.97 \times 10^{5}$ & 5.29 \\
$\mathbf{2 d}$ & -15.83 & $3.48 \times 10^{5}$ & 5.54 \\
$\mathbf{2 e}$ & -15.03 & $2.05 \times 10^{6}$ & 6.31 \\
\hline
\end{tabular}

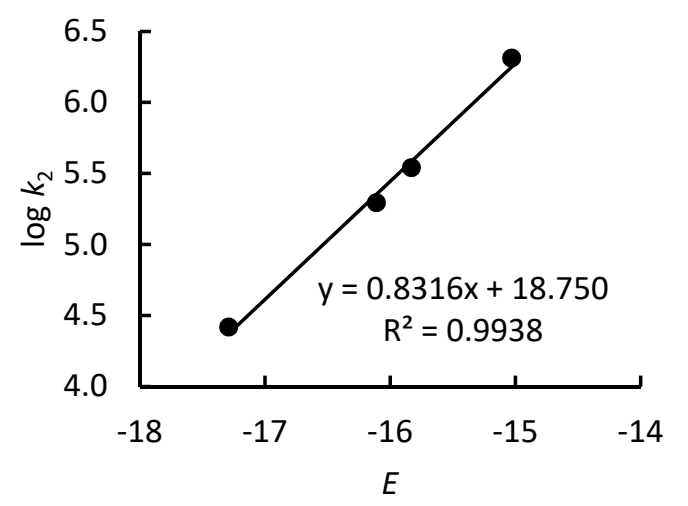

$N=22.55$

$S_{N}=0.83$ 


\section{Kinetics of reactions of thiophenolates with further electrophiles E1-E3}

- $\quad$ Kinetics of thiophenolate reactions with the 1,2-diaza-1,3-diene E1

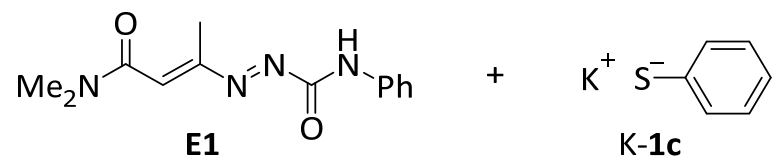

Table S59. 1,2-Diaza-1,3-diene E1 and potassium thiophenolate (K-1c) in DMSO (detection at $380 \mathrm{~nm}$ )

\begin{tabular}{llll}
\hline$[\mathrm{E} 1]_{0}, \mathrm{M}$ & {$[\mathbf{1 c}]_{0}, \mathrm{M}$} & {$[1 \mathrm{c}-\mathrm{H}]_{0}, \mathrm{M}$} & $k_{\mathrm{obs}}, \mathrm{s}^{-1}$ \\
\hline $1.79 \times 10^{-3}$ & $6.25 \times 10^{-5}$ & $5.00 \times 10^{-3}$ & 37.6 \\
$1.79 \times 10^{-3}$ & $1.25 \times 10^{-4}$ & $5.00 \times 10^{-3}$ & 79.6 \\
$1.79 \times 10^{-3}$ & $1.88 \times 10^{-4}$ & $5.00 \times 10^{-3}$ & 123 \\
$1.79 \times 10^{-3}$ & $2.50 \times 10^{-4}$ & $5.00 \times 10^{-3}$ & 173 \\
\hline
\end{tabular}

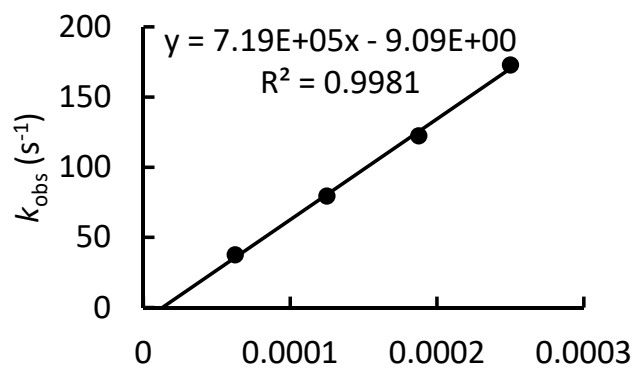

[1c] (M)

$k_{2}=7.19 \times 10^{5} \mathrm{M}^{-1} \mathrm{~s}^{-1}$

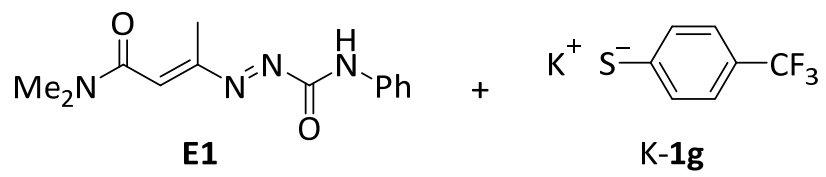

Table S60. 1,2-Diaza-1,3-diene E1 and potassium 4-(trifluoromethyl)thiophenolate (K-1g) in DMSO (detection at $380 \mathrm{~nm})$

\begin{tabular}{llll}
\hline$[\mathrm{E} 1]_{0}, \mathrm{M}$ & {$[\mathbf{1 g}]_{0}, \mathrm{M}$} & {$[\mathbf{1 g}-\mathrm{H}]_{0}, \mathrm{M}$} & $k_{\mathrm{obs}}, \mathrm{s}^{-1}$ \\
\hline $1.79 \times 10^{-3}$ & $4.86 \times 10^{-4}$ & $5.00 \times 10^{-3}$ & 43.1 \\
$1.79 \times 10^{-3}$ & $9.71 \times 10^{-4}$ & $5.00 \times 10^{-3}$ & 85.9 \\
$1.79 \times 10^{-3}$ & $1.46 \times 10^{-3}$ & $5.00 \times 10^{-3}$ & 131 \\
$1.79 \times 10^{-3}$ & $1.94 \times 10^{-3}$ & $5.00 \times 10^{-3}$ & 170 \\
\hline
\end{tabular}

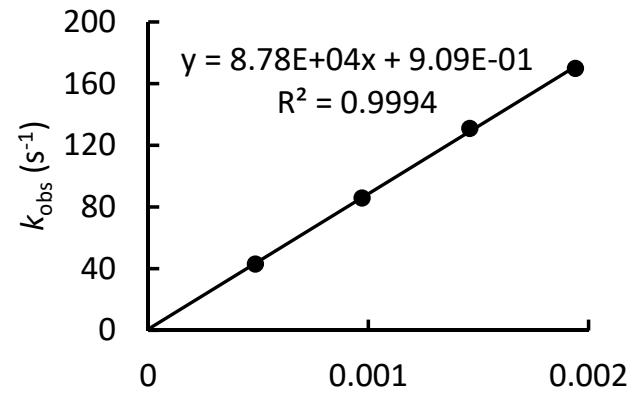

[1g] (M)

$k_{2}=8.78 \times 10^{4} \mathrm{M}^{-1} \mathrm{~s}^{-1}$ 
- $\quad$ Kinetics of thiophenolate reactions with the isothiocyanate E2<smiles>[R16][S-]c1ccc(C(F)(F)F)cc1</smiles>

Table S61 Isothiocyanate E2 and potassium 4-(trifluoromethyl)thiophenolate (K-1g) in DMSO (detection of increase at $440 \mathrm{~nm}$ )

\begin{tabular}{llll}
\hline$[E 2]_{0}, M$ & {$[1 \mathrm{~g}]_{0}, \mathrm{M}$} & {$[\mathbf{1 g}-\mathrm{H}]_{0}, \mathrm{M}$} & $k_{\mathrm{obs}}, \mathrm{s}^{-1}$ \\
\hline $5.39 \times 10^{-4}$ & $4.86 \times 10^{-4}$ & $5.00 \times 10^{-3}$ & 314 \\
$5.39 \times 10^{-4}$ & $9.71 \times 10^{-4}$ & $5.00 \times 10^{-3}$ & 602 \\
$5.39 \times 10^{-4}$ & $1.46 \times 10^{-3}$ & $5.00 \times 10^{-3}$ & 886 \\
$5.39 \times 10^{-4}$ & $1.94 \times 10^{-3}$ & $5.00 \times 10^{-3}$ & $1.14 \times 10^{3}$ \\
\hline
\end{tabular}

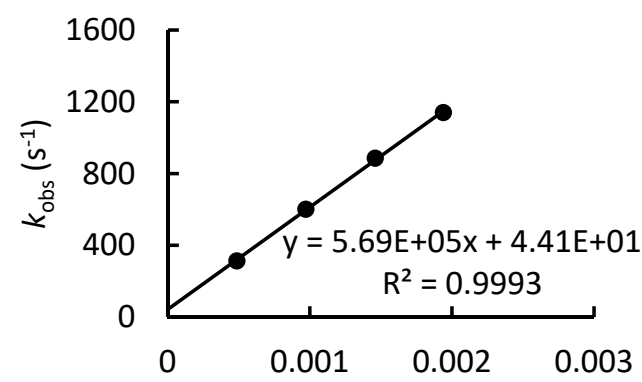

[1g] (M)

$k_{2}=5.69 \times 10^{5} \mathrm{M}^{-1} \mathrm{~s}^{-1}$

- $\quad$ Kinetics of thiophenolate reactions with the isothiocyanate E3

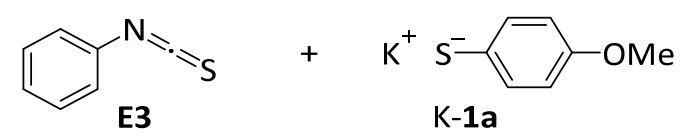

Table S62. Ph-NCS (E3) and potassium 4-methoxy-thiophenolate (K-1a) in DMSO (detection of increase at 320 $\mathrm{nm})$

\begin{tabular}{llll}
\hline$[E 3]_{0}, M$ & {$[1 \mathrm{a}]_{0}, \mathrm{M}$} & {$[\mathbf{1 a}-\mathrm{H}]_{0}, \mathrm{M}$} & $k_{\mathrm{obs}}, \mathrm{s}^{-1}$ \\
\hline $5.93 \times 10^{-3}$ & $6.00 \times 10^{-4}$ & 0 & 316 \\
$1.19 \times 10^{-2}$ & $6.00 \times 10^{-4}$ & 0 & 603 \\
$1.78 \times 10^{-2}$ & $6.00 \times 10^{-4}$ & 0 & 886 \\
$2.37 \times 10^{-2}$ & $6.00 \times 10^{-4}$ & 0 & $1.16 \times 10^{3}$ \\
\hline
\end{tabular}

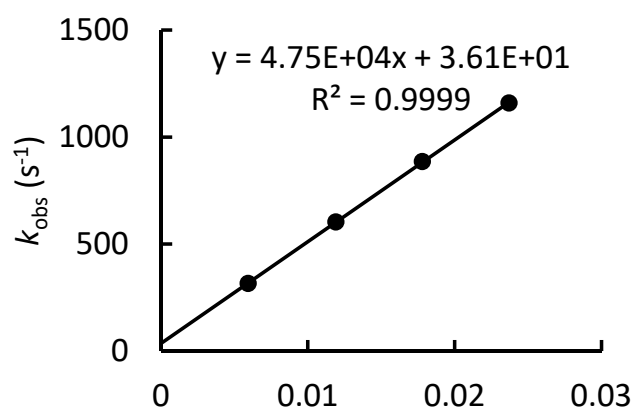

$k_{2}=4.75 \times 10^{4} \mathrm{M}^{-1} \mathrm{~s}^{-1}$

[E3] (M)

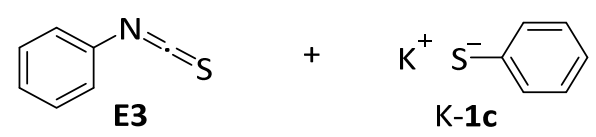

Table S63. Ph-NCS (E3) and potassium thiophenolate (K-1c) in DMSO (detection of increase at $326 \mathrm{~nm}$ )

\begin{tabular}{llll}
\hline$[\text { E3 }]_{0}, \mathrm{M}$ & {$[\mathbf{1 c}]_{0}, \mathrm{M}$} & {$[\mathbf{1 c}-\mathrm{H}]_{0}, \mathrm{M}$} & $k_{\mathrm{obs}, \mathrm{s}^{-1}}$ \\
\hline $5.93 \times 10^{-3}$ & $6.00 \times 10^{-4}$ & 0 & 55.3 \\
$1.19 \times 10^{-2}$ & $6.00 \times 10^{-4}$ & 0 & 83.2 \\
$1.78 \times 10^{-2}$ & $6.00 \times 10^{-4}$ & 0 & 113 \\
$2.37 \times 10^{-2}$ & $6.00 \times 10^{-4}$ & 0 & 142 \\
\hline
\end{tabular}

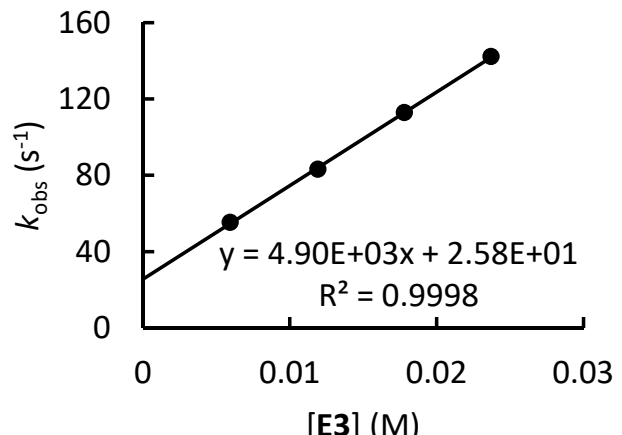

$k_{2}=4.90 \times 10^{3} \mathrm{M}^{-1} \mathrm{~s}^{-1}$ 


\section{Copies of NMR Spectra}
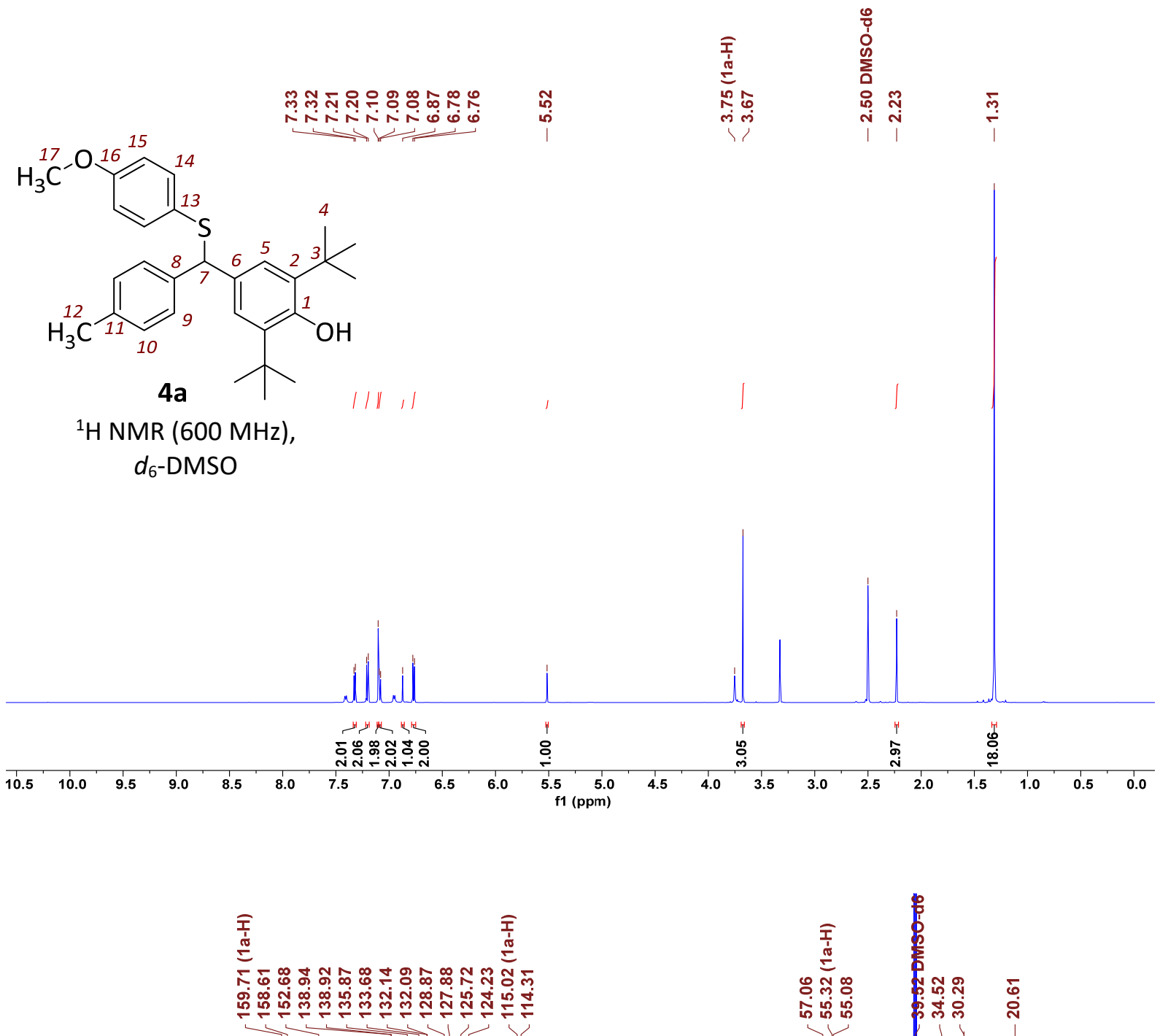

$4 a$

${ }^{13} \mathrm{C}\left\{{ }^{1} \mathrm{H}\right\}$ NMR (151

$\mathrm{MHz})$,

$\begin{array}{llllllllllll}210 & 200 & 190 & 180 & 170 & 160 & 150 & 140 & 130 & 120 & 110 \begin{array}{r}100 \\ \mathrm{f} 1(\mathrm{ppm})\end{array}\end{array}$ 


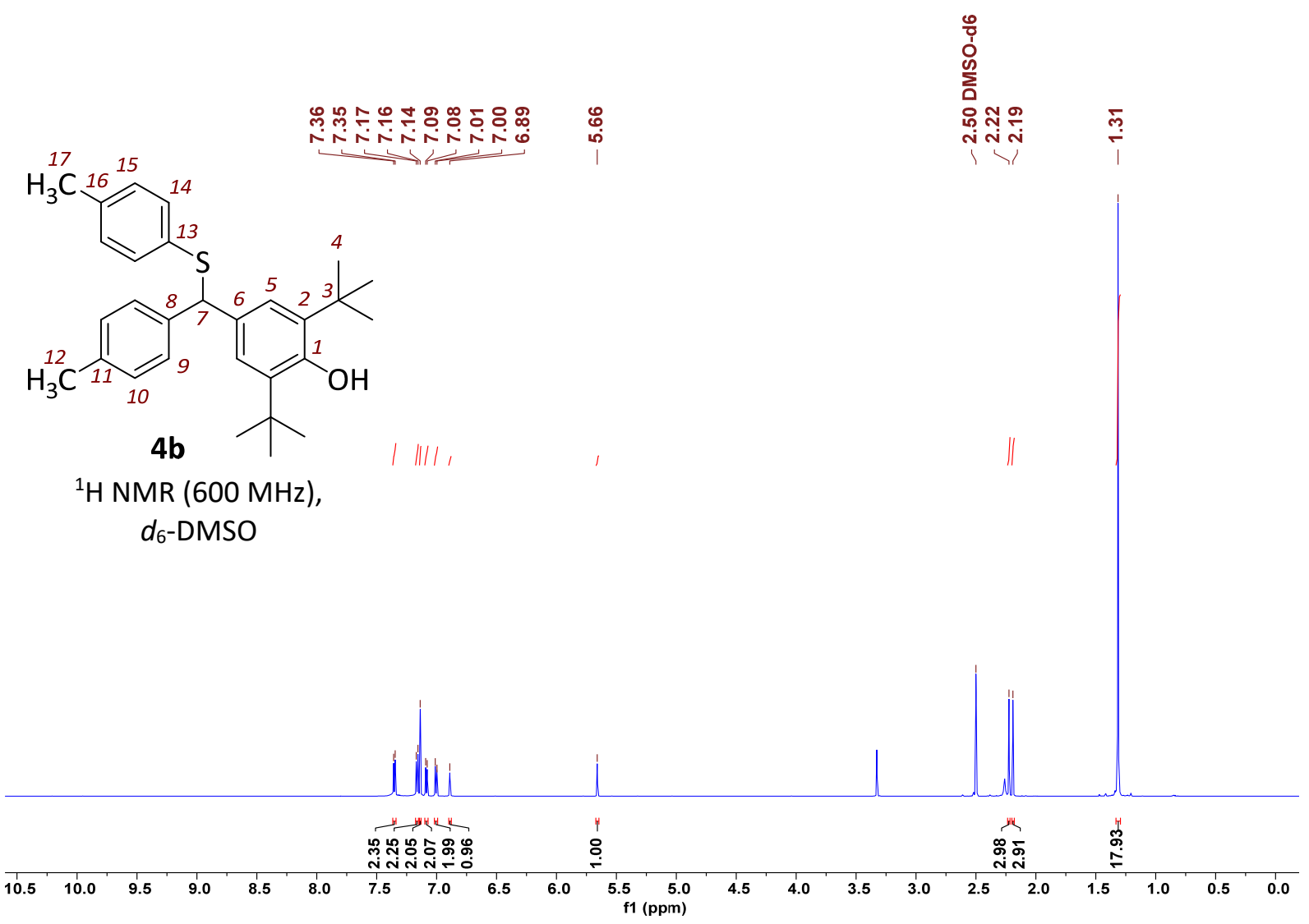

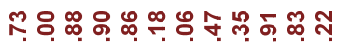
نิ่

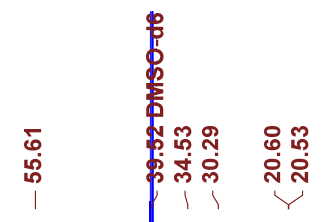

4b

${ }^{13} \mathrm{C}\left\{{ }^{1} \mathrm{H}\right\}$ NMR (151 MHz), $d_{6}$-DMSO

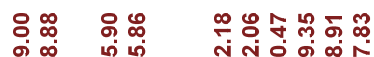

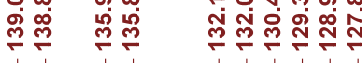

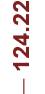
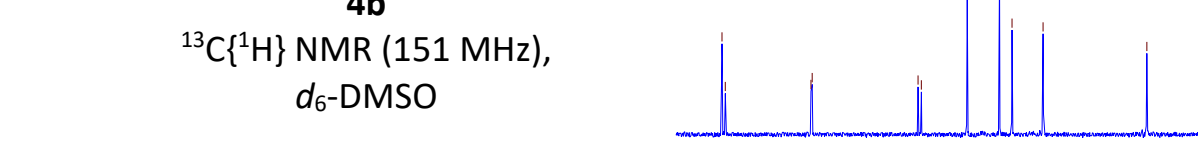

$\begin{array}{lllllllll}140 & 138 & 136 & 134 & 132 & 130 & 128 & 126 & 124\end{array}$

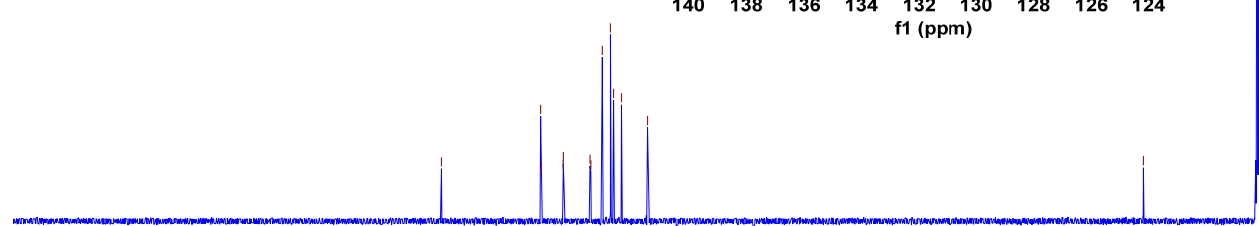

कां

\begin{tabular}{rllllllllll}
\hline 210 & 200 & 190 & 180 & 170 & 160 & 150 & 140 & 130 & 120 & 110 \\
$\mathrm{f} 1(\mathrm{ppm})$
\end{tabular}

$90 \quad 80$

60

$\begin{array}{llllll}50 & 40 & 30 & 20 & 10 & 0\end{array}$ 


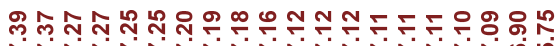

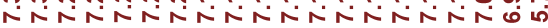<smiles>Cc1ccc(C(Sc2ccccc2)c2cc(C(C)(C)C)c(O)c(C(C)(C)C)c2)cc1</smiles>

${ }^{1} \mathrm{H}$ NMR $(600 \mathrm{MHz})$,

$d_{6}$-DMSO

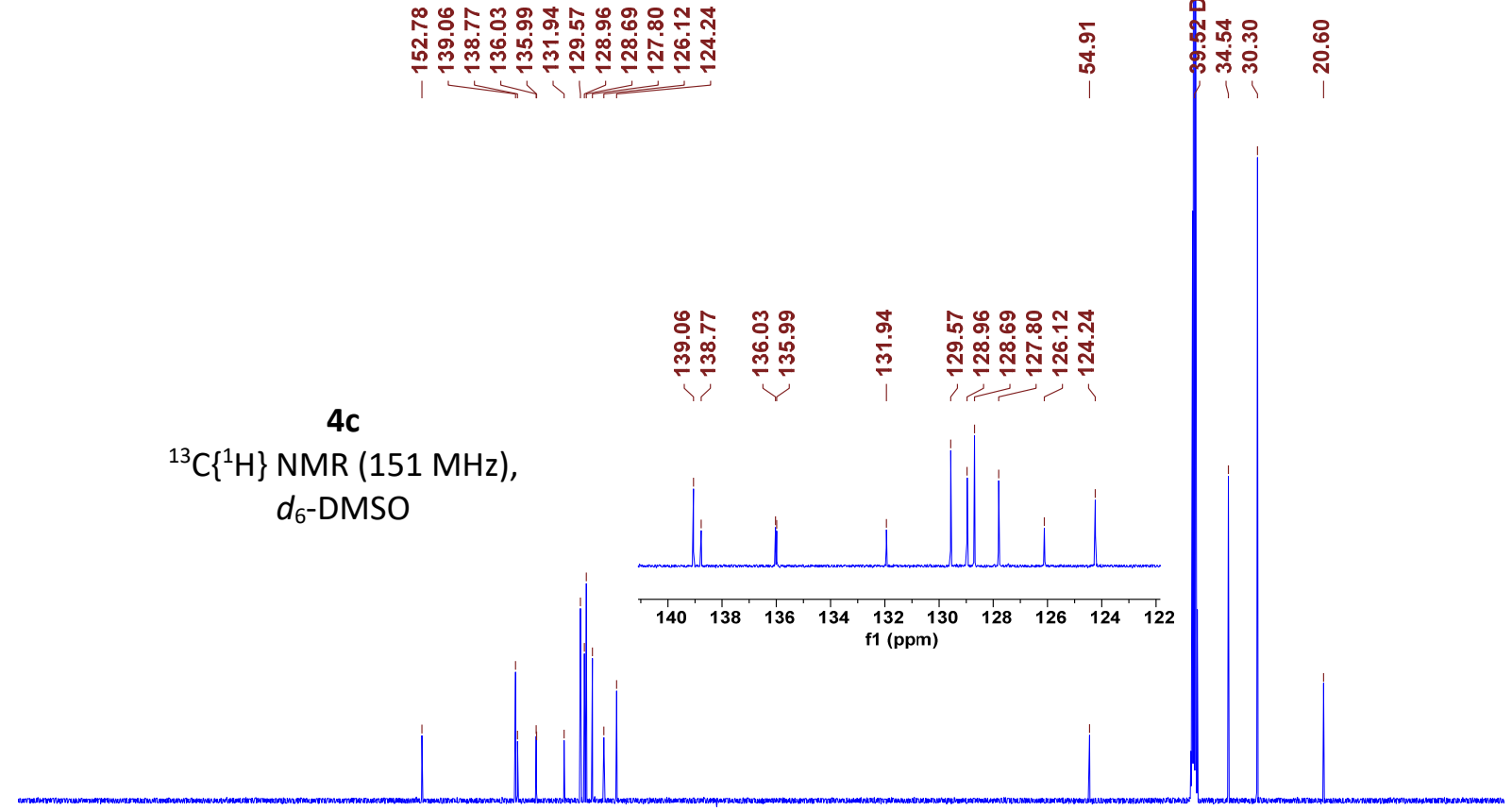

$\begin{array}{lllllllllllllllllllllllllllll}210 & 200 & 190 & 180 & 170 & 160 & 150 & 140 & 130 & 120 & 110 & 100 & 90 & 80 & 70 & 60 & 50 & 40 & 30 & 20 & 10 & 0 & \end{array}$ 

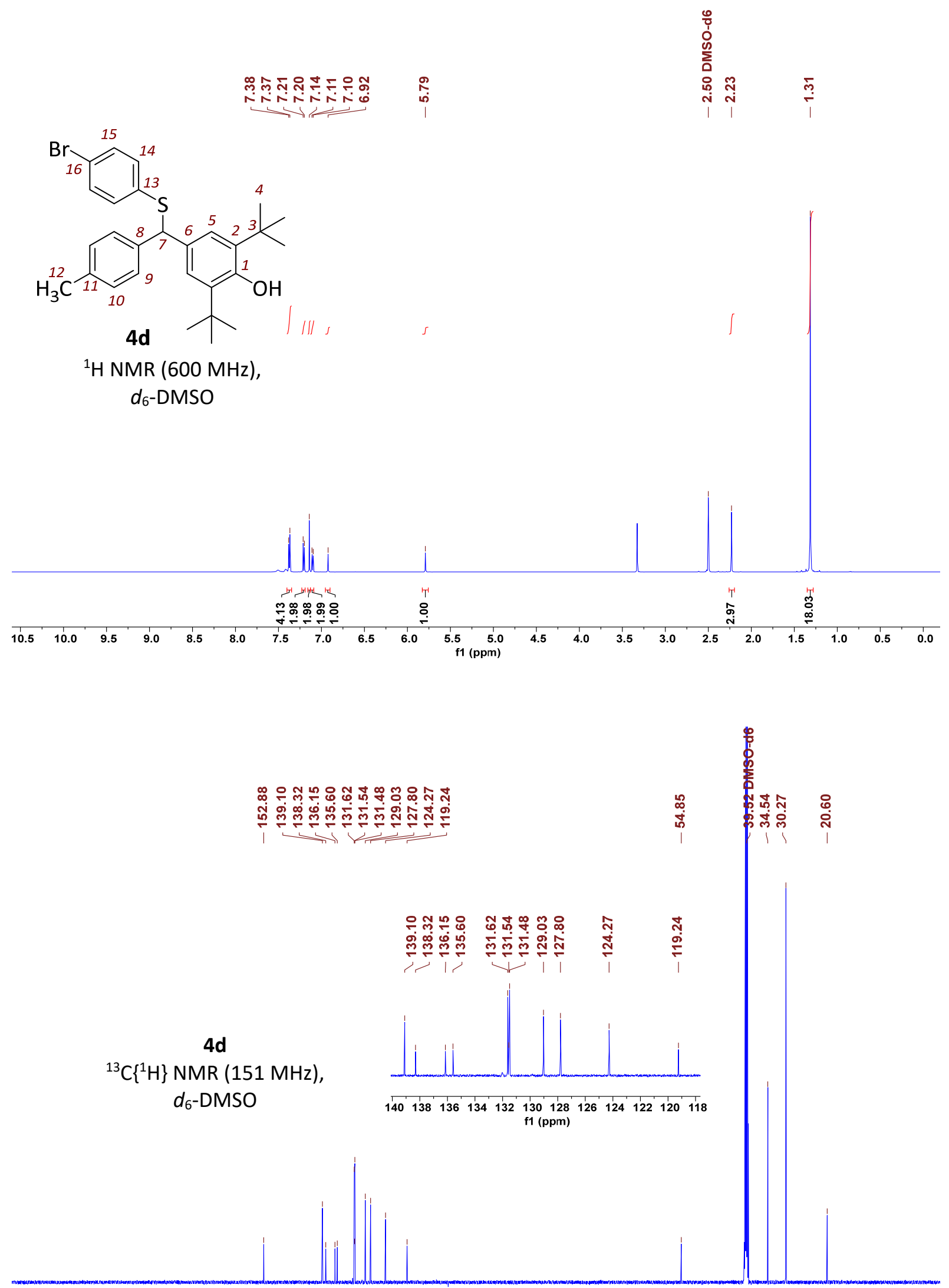

$\begin{array}{lllllllllllllllllllllllllllll}210 & 200 & 190 & 180 & 170 & 160 & 150 & 140 & 130 & 120 & 110 & 100 & 90 & 80 & 70 & 60 & 50 & 40 & 30 & 20 & 10 & 0\end{array}$ 


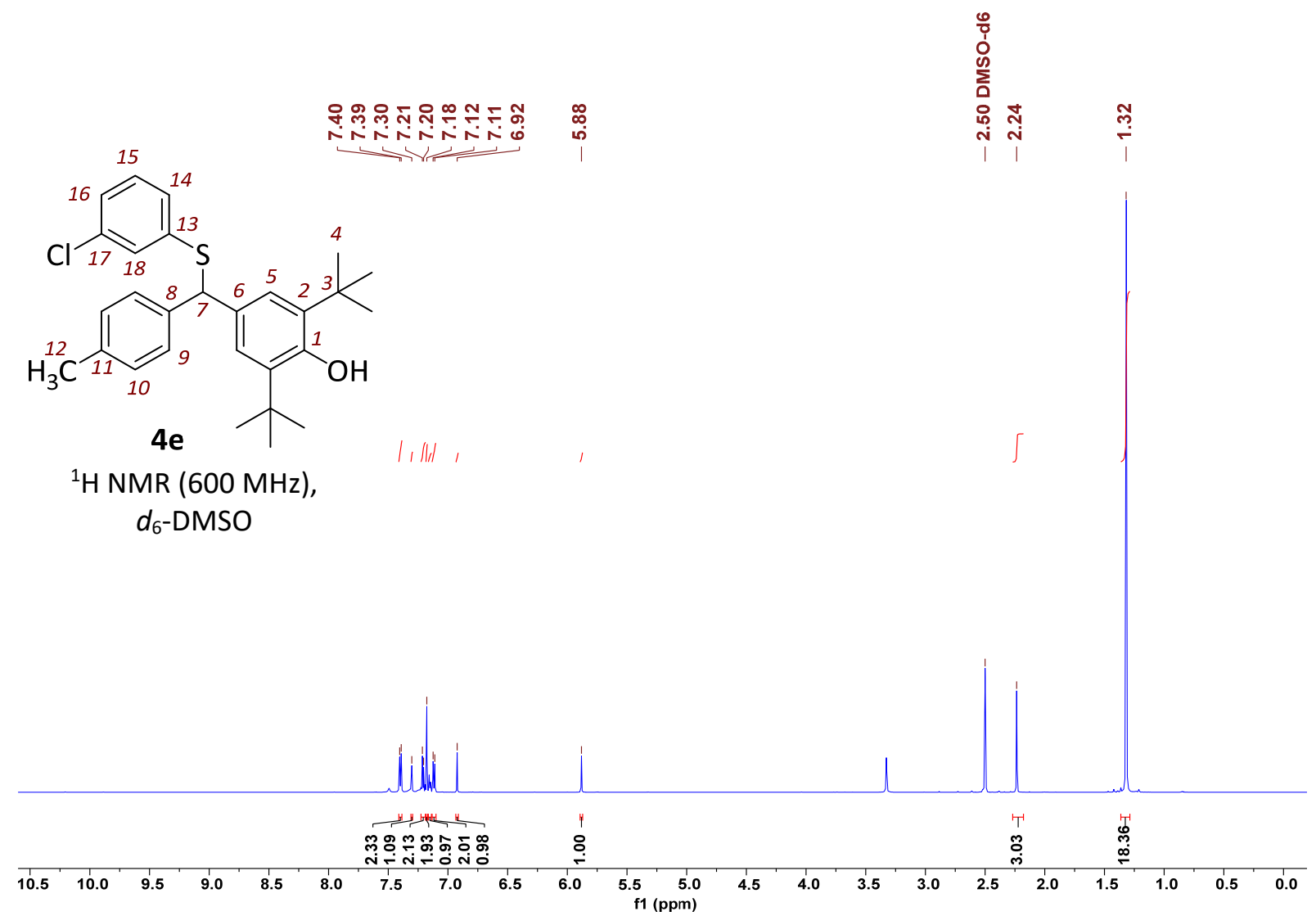

4 e

${ }^{13} \mathrm{C}\left\{{ }^{1} \mathrm{H}\right\}$ NMR $(151 \mathrm{MHz})$,

$d_{6}$-DMSO

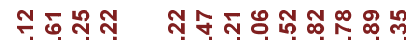

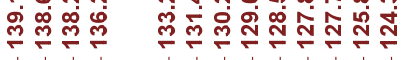

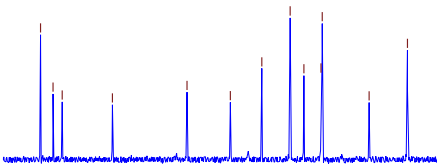
$\begin{array}{lllllllllll}140 & 138 & 136 & 134 & 132 & 130 & 128 & 126 & 124 \\ \mathrm{f} 1(\mathrm{ppm}) & & & & & & \end{array}$

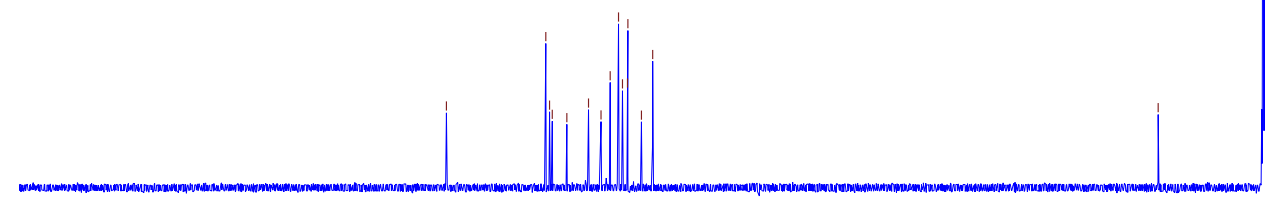

210

$\begin{array}{llllllllll}200 & 190 & 180 & 170 & 160 & 150 & 140 & 130 & 120 & 110 \begin{array}{r}100 \\ \mathrm{f} 1(\mathrm{ppm})\end{array}\end{array}$

$90 \quad 80$

$70 \quad 60$

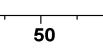

40

$\begin{array}{lll}20 & 10\end{array}$ 

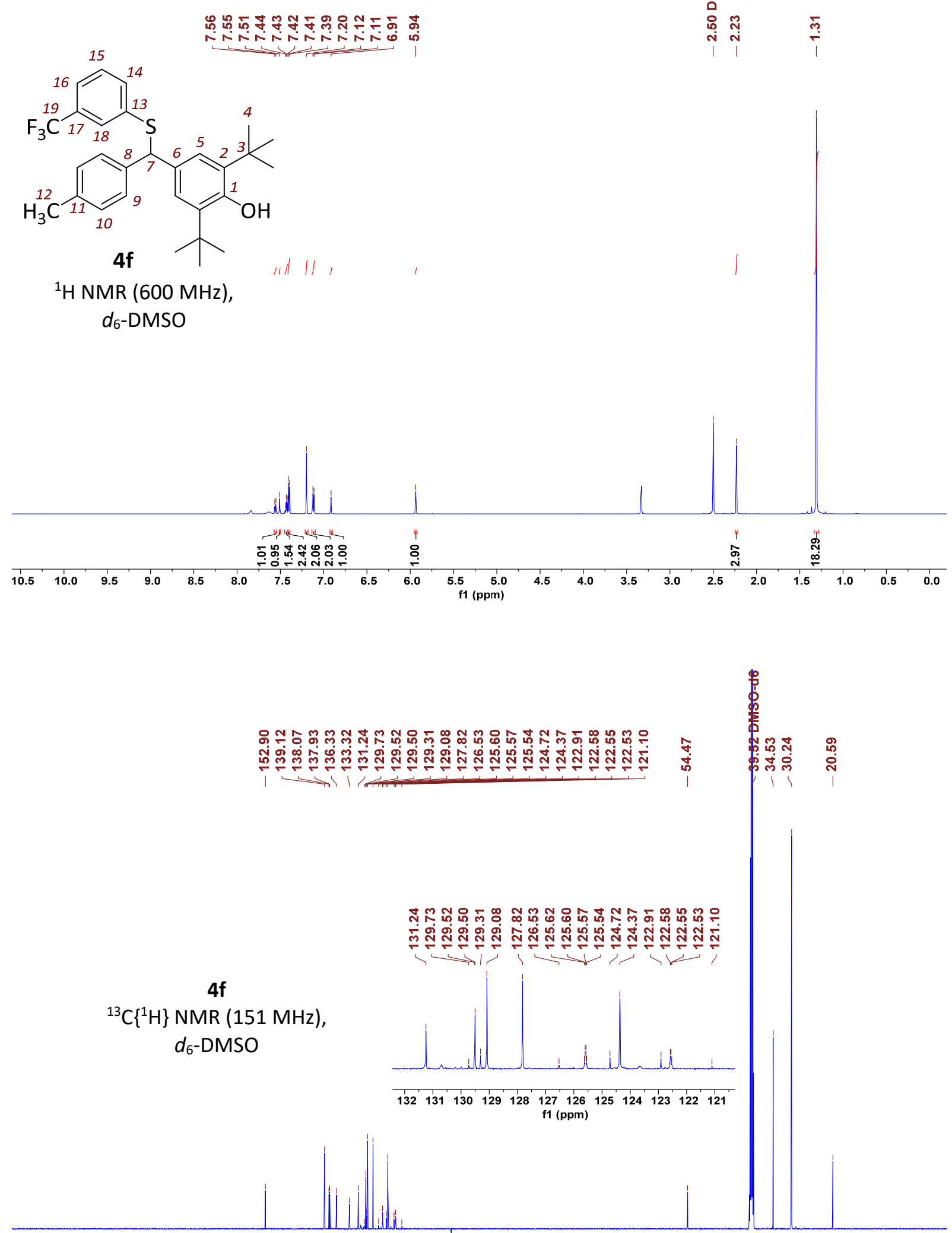

$\begin{array}{llllllllllllllllllllllllllllll}210 & 200 & 190 & 180 & 170 & 160 & 150 & 140 & 130 & 120 & 110 & 100 & 90 & 80 & 70 & 60 & 50 & 40 & 30 & 20 & 10 & 0 & \end{array}$ 


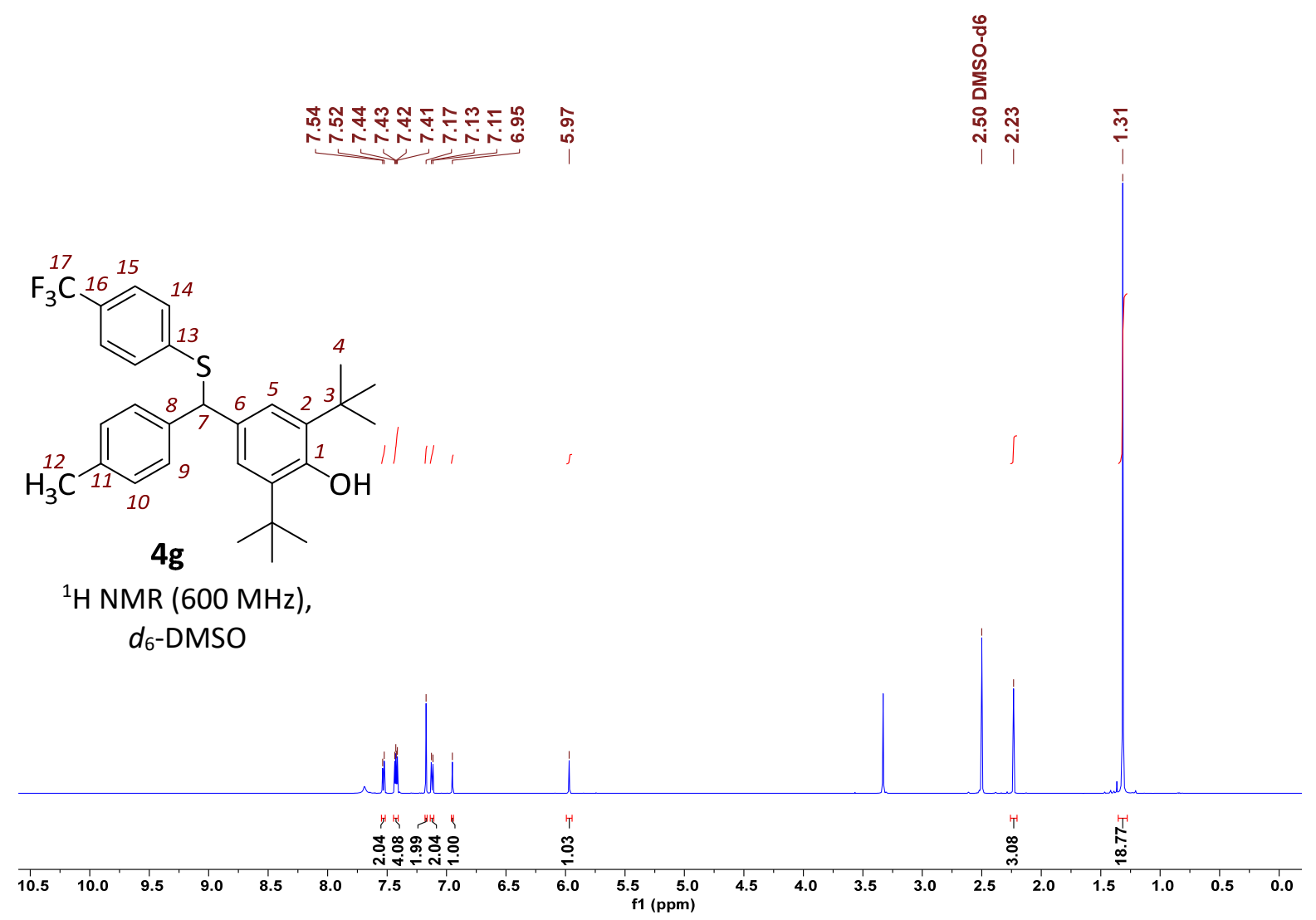

๓

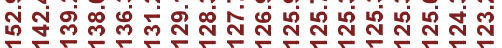

$4 \mathrm{~g}$

${ }^{13} \mathrm{C}\left\{{ }^{1} \mathrm{H}\right\}$ NMR $(151 \mathrm{MHz})$,

$d_{6}$-DMSO

\begin{tabular}{lllllllllllllllllllllllllllll}
\hline 210 & 200 & 190 & 180 & 170 & 160 & 150 & 140 & 130 & 120 & 110 & 100 & 90 & 80 & 70 & 60 & 50 & 40 & 30 & 20 & 10 & 0
\end{tabular} 

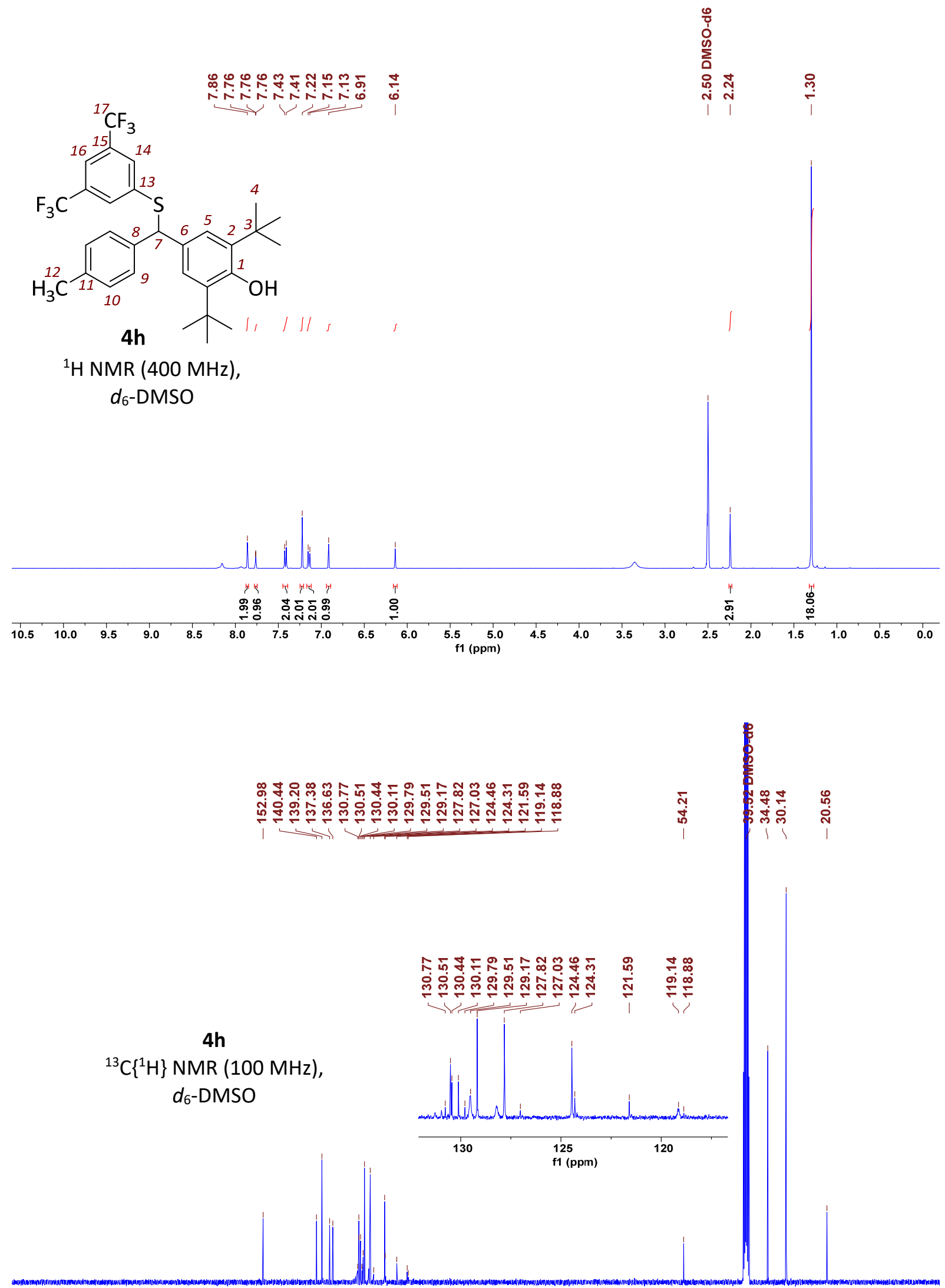

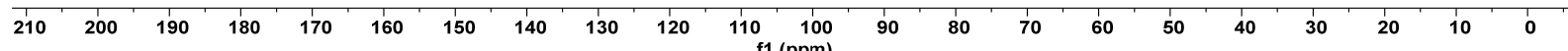




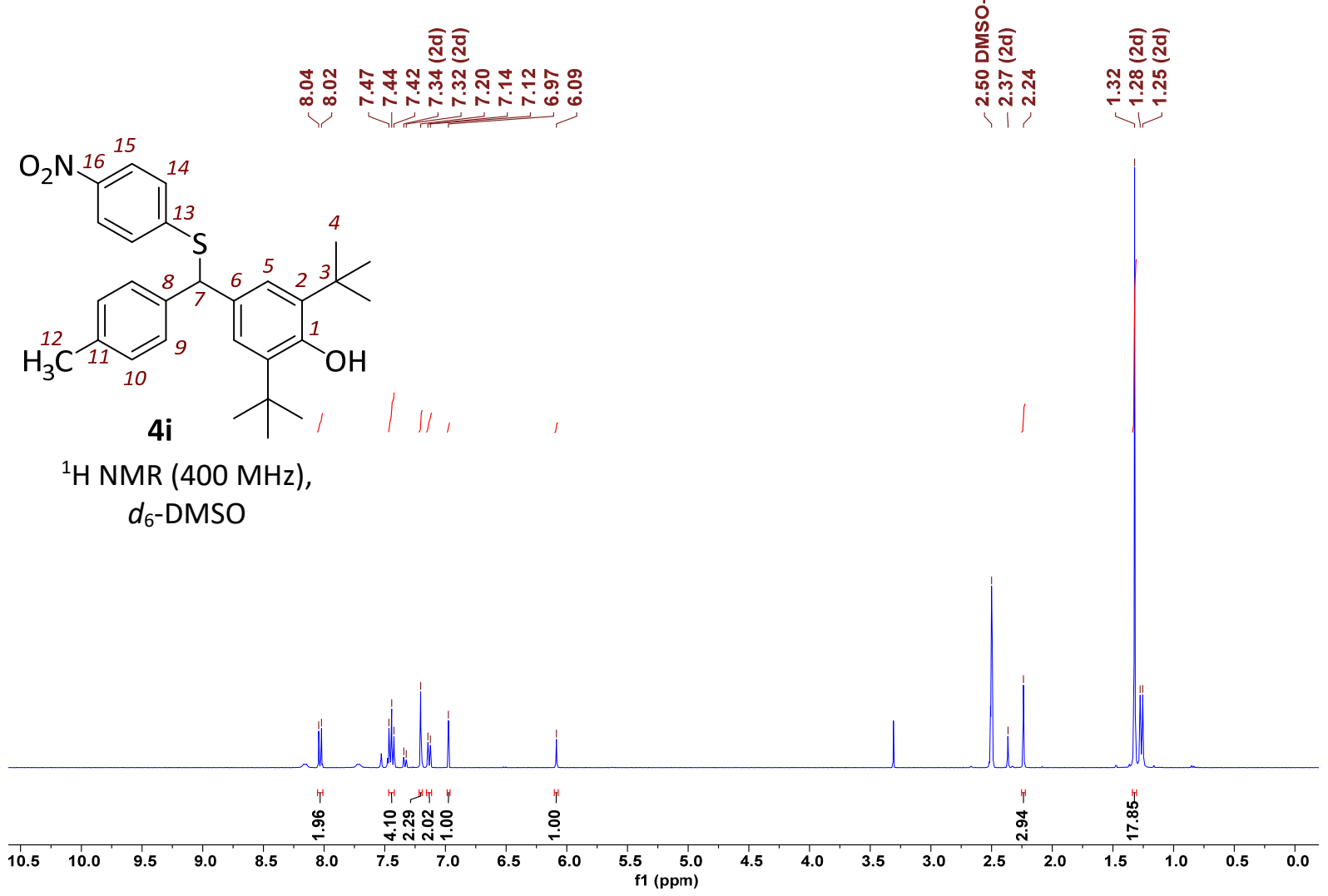

ㅇํㅇํำ

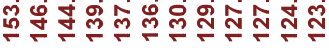

4i (+ residual 2d)

${ }^{13} \mathrm{C}\left\{{ }^{1} \mathrm{H}\right\}$ NMR (100 MHz),

$d_{6}$-DMSO

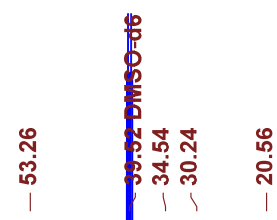

\begin{tabular}{llllllllllll}
\hline 210 & 200 & 190 & 180 & 170 & 160 & 150 & 140 & 130 & 120 & 110 & 100
\end{tabular}

$90 \quad 80 \quad 70 \quad 60$ 


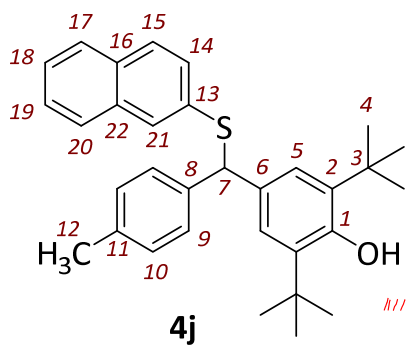

${ }^{1} \mathrm{H}$ NMR $(600 \mathrm{MHz})$,

$$
d_{6} \text {-DMSO }
$$

$4 j$

${ }^{13} \mathrm{C}\left\{{ }^{1} \mathrm{H}\right\}$ NMR (151 MHz), $d_{6}$-DMSO
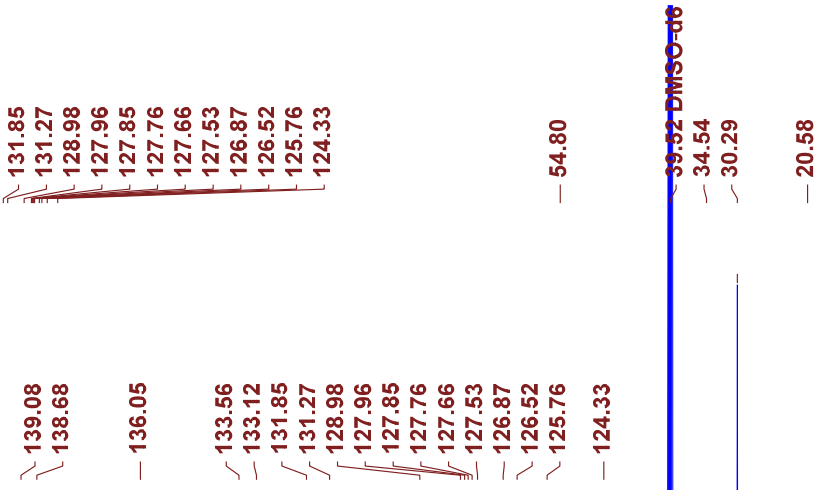


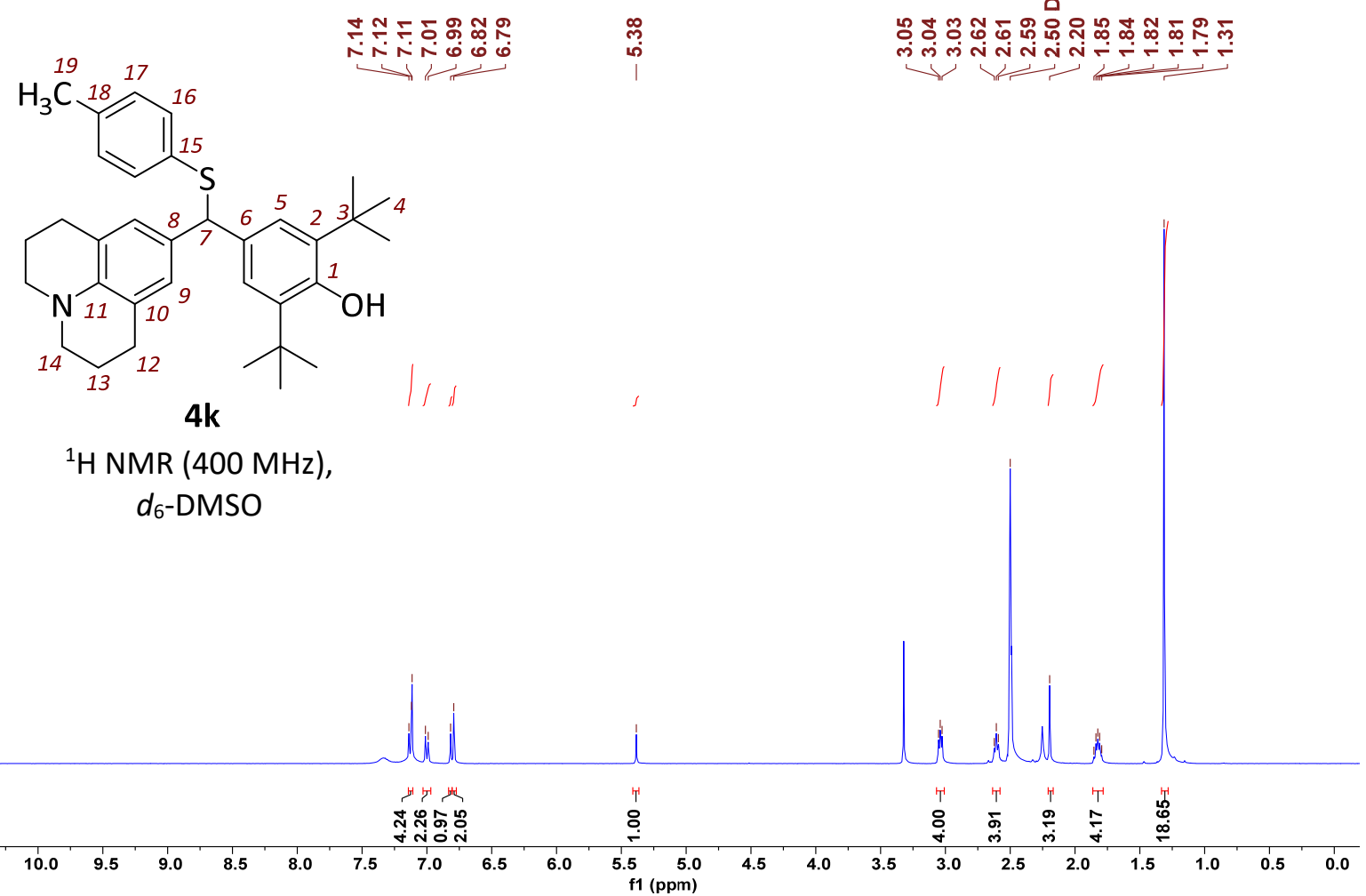

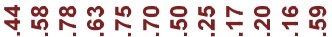

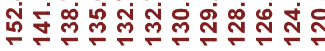

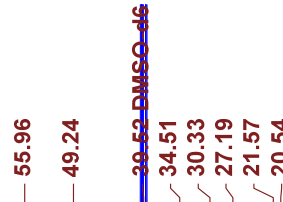

4k

${ }^{13} \mathrm{C}\left\{{ }^{1} \mathrm{H}\right\}$ NMR (101 MHz),

$d_{6}$-DMSO

$\begin{array}{llllllllllll}210 & 200 & 190 & 180 & 170 & 160 & 150 & 140 & 130 & 120 & 110 & 100\end{array}$

$90 \quad 80 \quad 70 \quad 60$

$50 \quad 40 \quad 30$

20 
<smiles>Cc1ccc(S)cc1</smiles><smiles>C=CCSC(c1ccc(N(C)C)cc1)c1cc(C(C)(C)C)c(O)c(C(C)(C)C)c1</smiles>

41

${ }^{1} \mathrm{H}$ NMR (400 MHz), $d_{6}$-DMSO

41

${ }^{13} \mathrm{C}\left\{{ }^{1} \mathrm{H}\right\}$ NMR (101 MHz),

$d_{6}$-DMSO

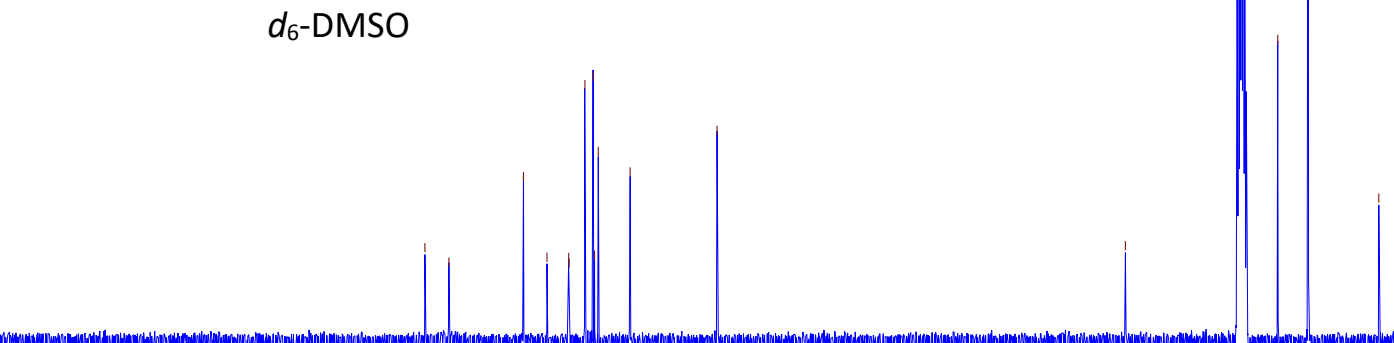




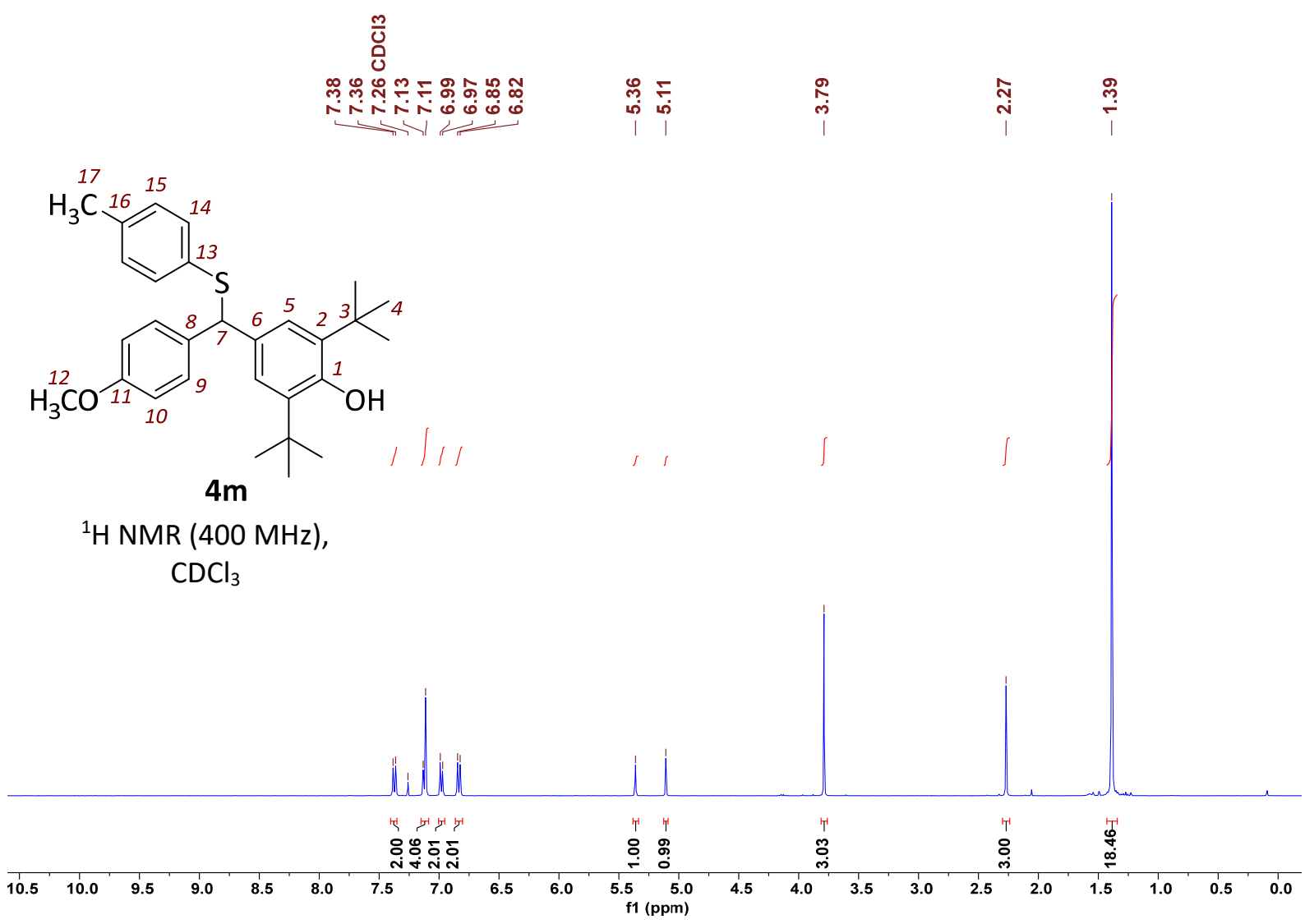

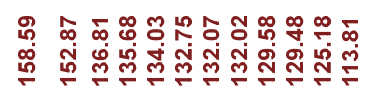

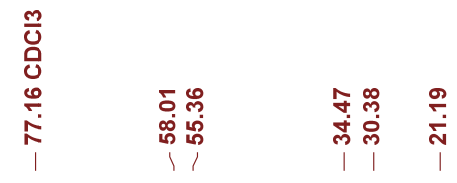

$4 \mathrm{~m}$

${ }^{13} \mathrm{C}\left\{{ }^{1} \mathrm{H}\right\}$ NMR (101 MHz),

$\mathrm{CDCl}_{3}$

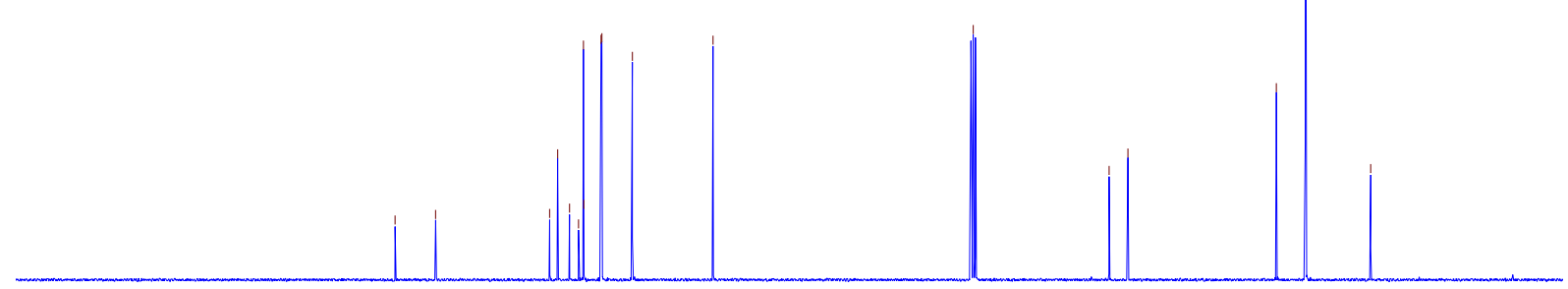

\begin{tabular}{llllllllllllllllllllll}
\hline 210 & 200 & 190 & 180 & 170 & 160 & 150 & 140 & 130 & 120 & 110 & 100 & 90 & 80 & 70 & 60 & 50 & 40 & 30 & 20 & 10 & 0
\end{tabular} 


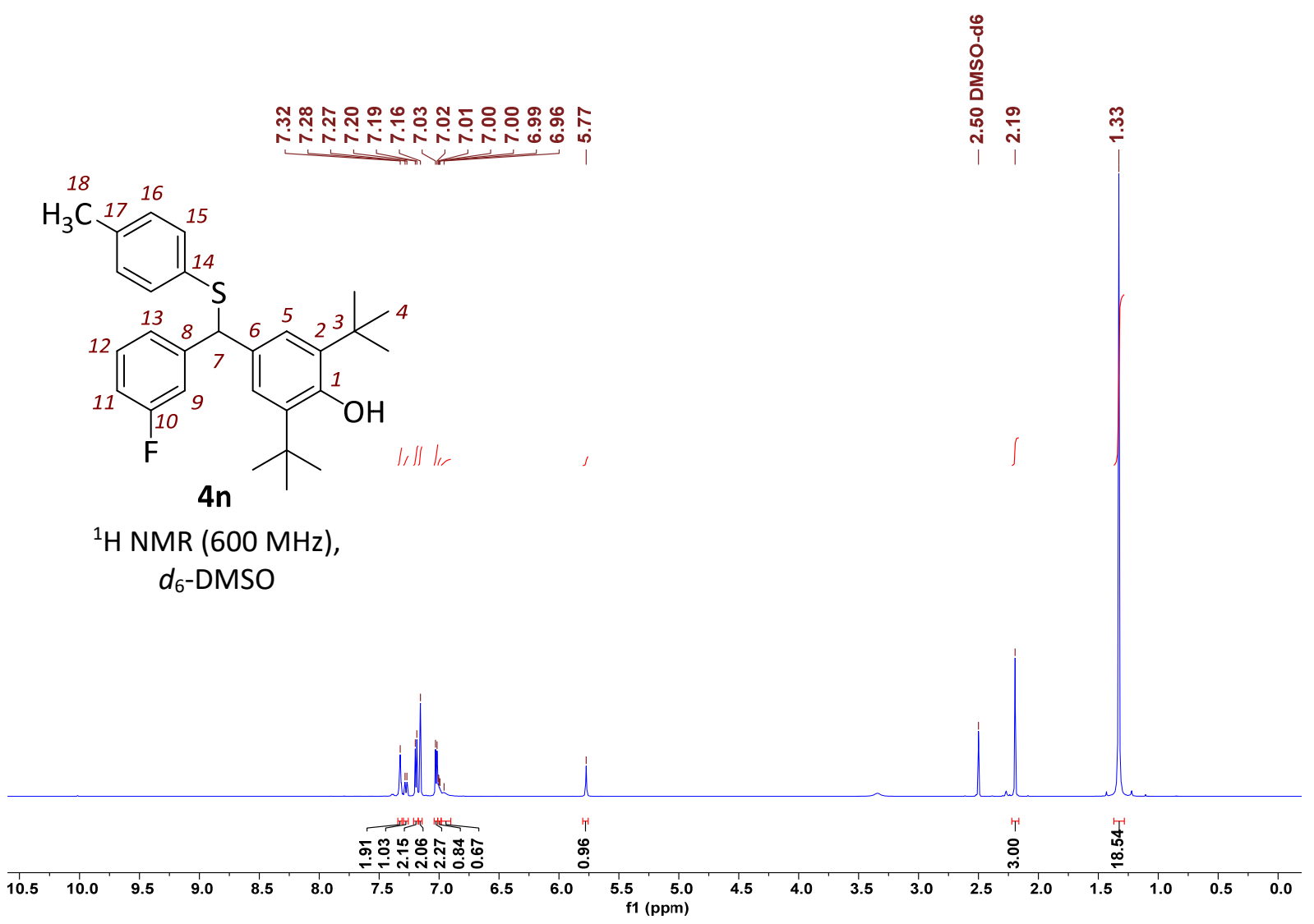

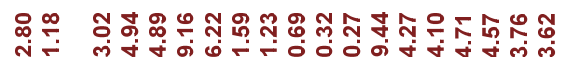

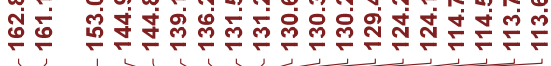
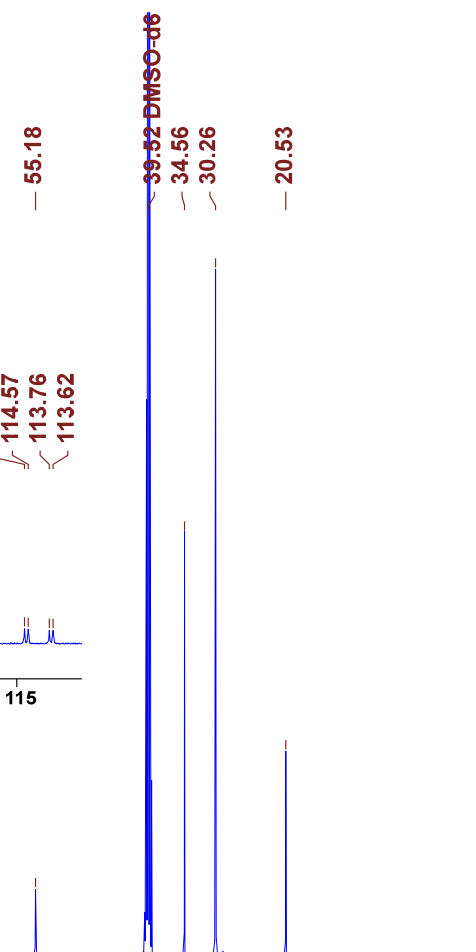

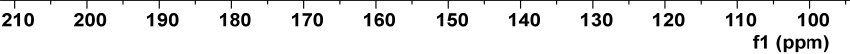

$90 \quad 80$

4n

${ }^{13} \mathrm{C}\left\{{ }^{1} \mathrm{H}\right\}$ NMR (151 MHz),

$d_{6}$-DMSO

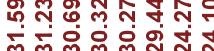

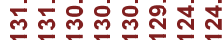

단ำ

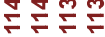
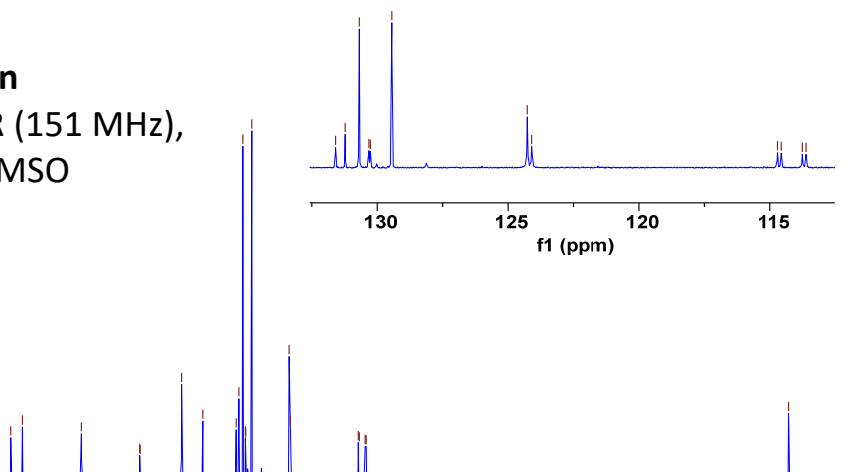
<smiles>Cc1ccc(SC(c2ccc([N+](=O)[O-])cc2)c2cc(C(C)(C)C)c(O)c(C(C)(C)C)c2)cc1</smiles>

${ }^{1} \mathrm{H}$ NMR (400 MHz),

$d_{6}$-DMSO

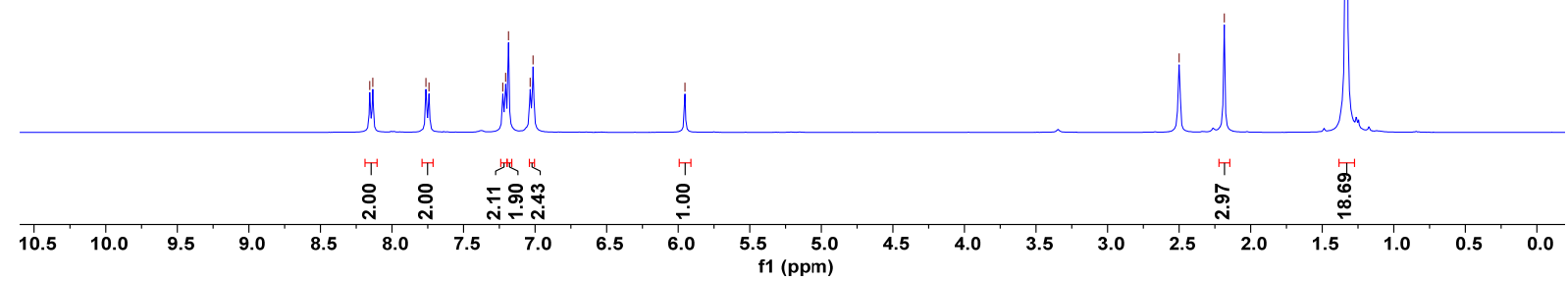

40

${ }^{13} \mathrm{C}\left\{{ }^{1} \mathrm{H}\right\}$ NMR (151 MHz),

$d_{6}$-DMSO

א్లో

站
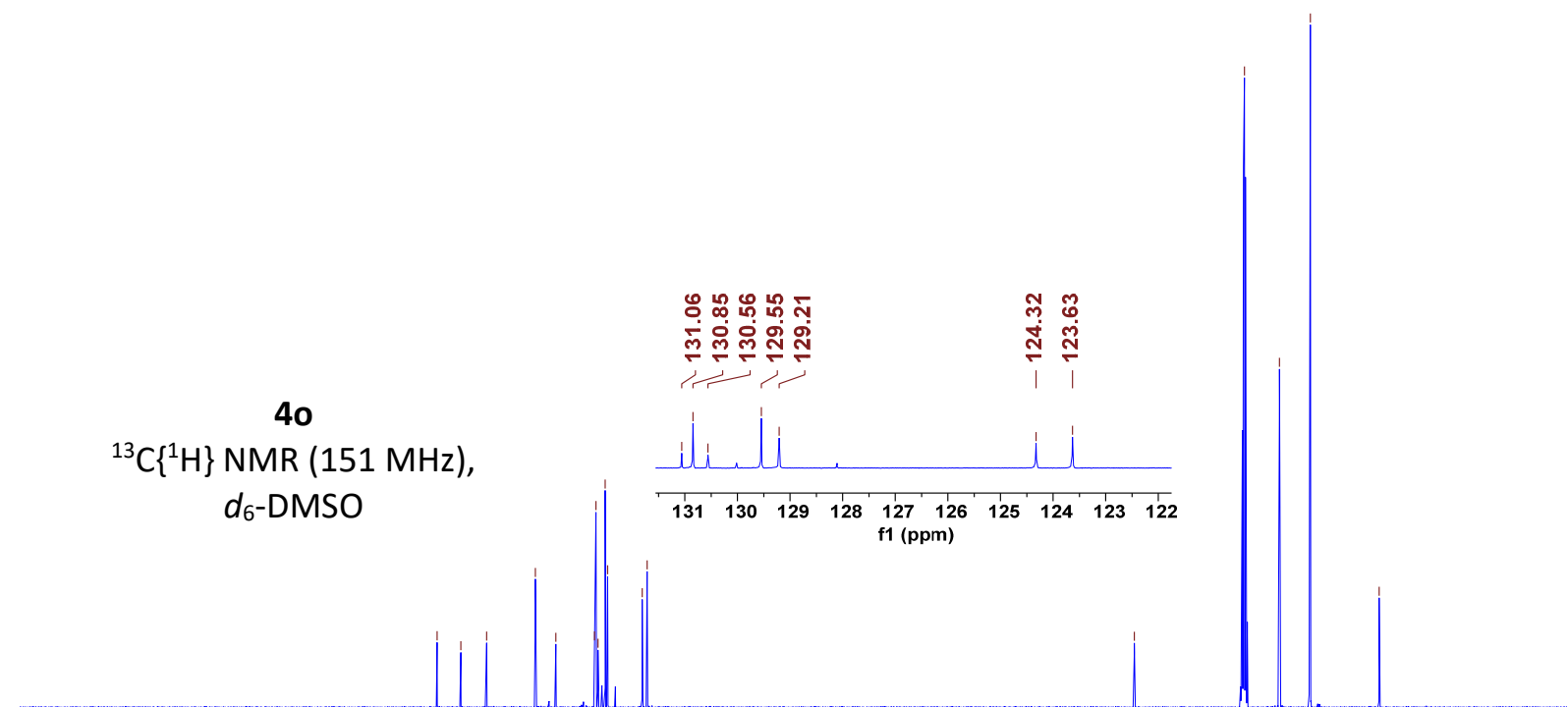

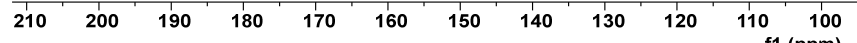

$90 \quad 80 \quad 70 \quad 60$

50

$30 \quad 20 \quad 10 \quad 0$ 


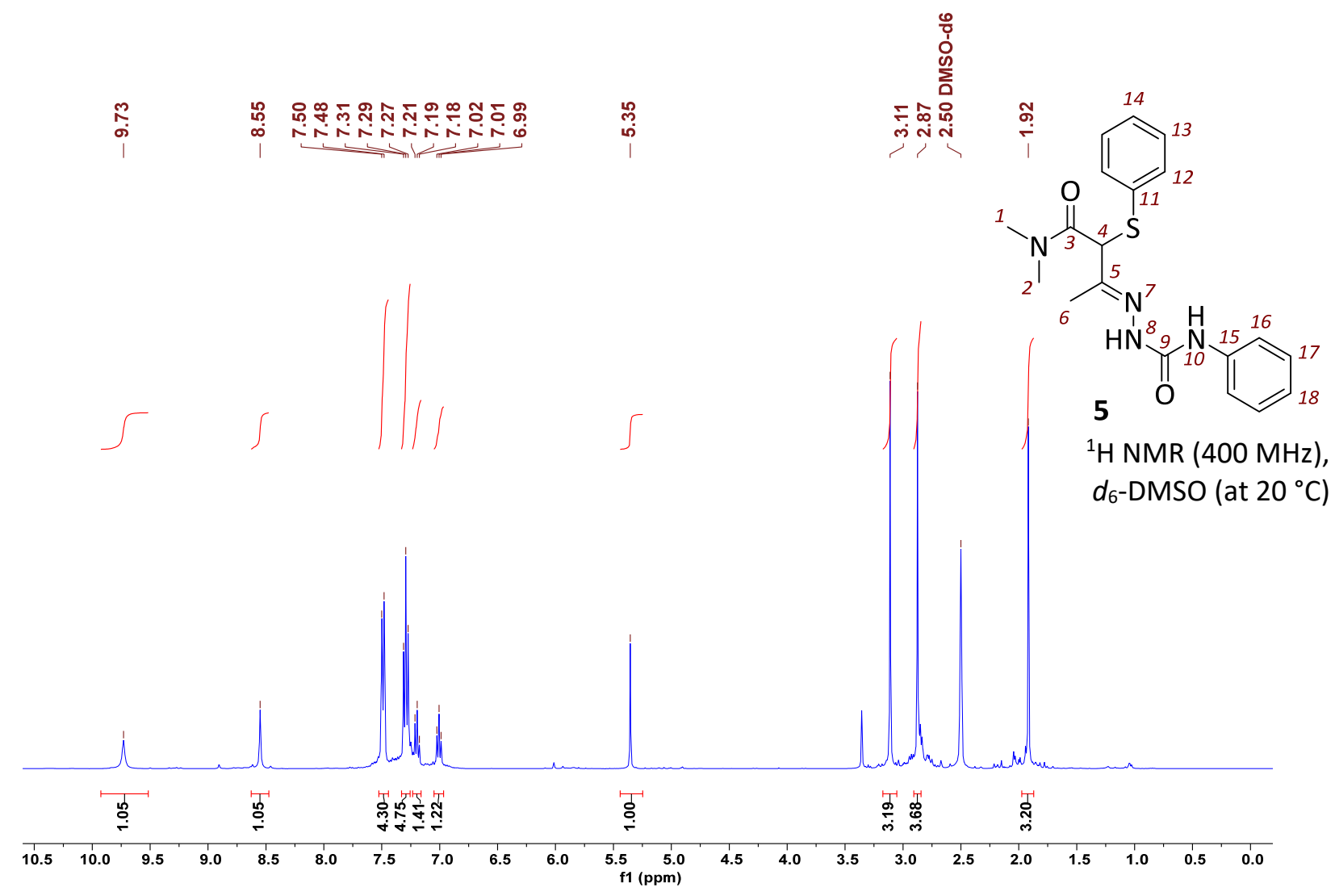

5

${ }^{13} \mathrm{C}\left\{{ }^{1} \mathrm{H}\right\}$ NMR (101 MHz), $d_{6}$-DMSO (at $80^{\circ} \mathrm{C}$ )

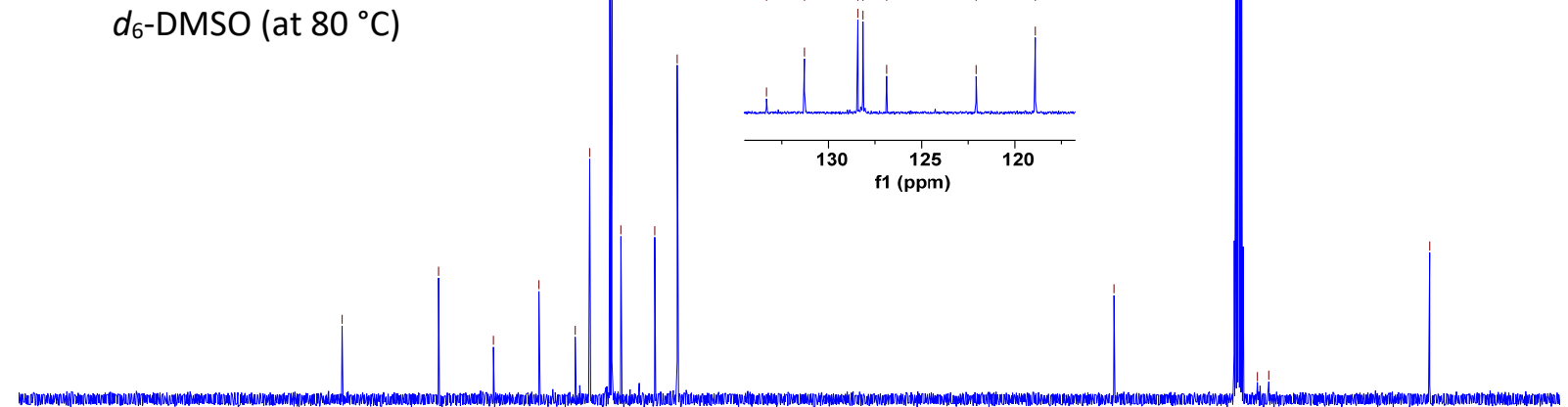




\section{References}

(S1) Hansch, C.; Leo, A.; Hoekman, D. Exploring QSAR - Hydrophobic, Electronic, and Steric Constants. ACS Professional Reference Book; American Chemical Society; Washington, DC, 1995.

(S2) Bordwell, F. G.; Hughes, D. L. Thiol Acidities and Thiolate Ion Reactivities toward Butyl Chloride in Dimethyl Sulfoxide Solution. The Question of Curvature in Brønsted Plots. J. Org. Chem. 1982, 47, 3224-3232.

(S3) Mayer, R. J.; Breugst, M.; Hampel, N.; Ofial, A. R.; Mayr, H. Ambident Reactivity of Phenolate Anions Revisited: A Quantitative Approach to Phenolate Reactivities. J. Org. Chem. 2019, 84, 88378858.

(S4) Richter, D.; Hampel, N.; Singer, T.; Ofial, A. R.; Mayr, H. Synthesis and Characterization of Novel Quinone Methides: Reference Electrophiles for the Construction of Nucleophilicity Scales. Eur. J. Org. Chem. 2009, 3203-3211. 